\title{
Discrepancies in Self- and Informant-Reports of Personality Pathology: Examining the DSM-5 Section III Trait Model
}

\section{Loading required packages}

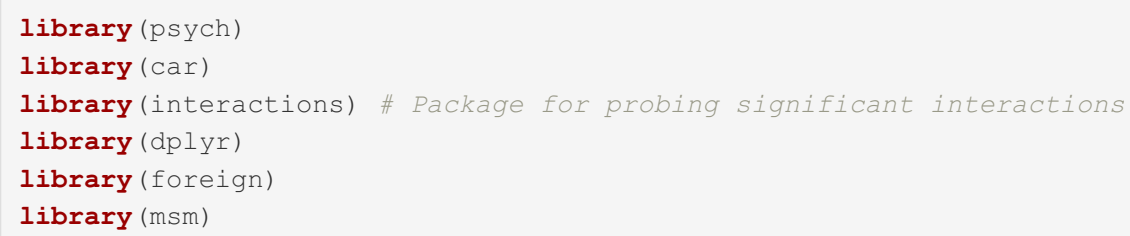

\section{Creating the GPPC (General Personality Pathology Composite)}

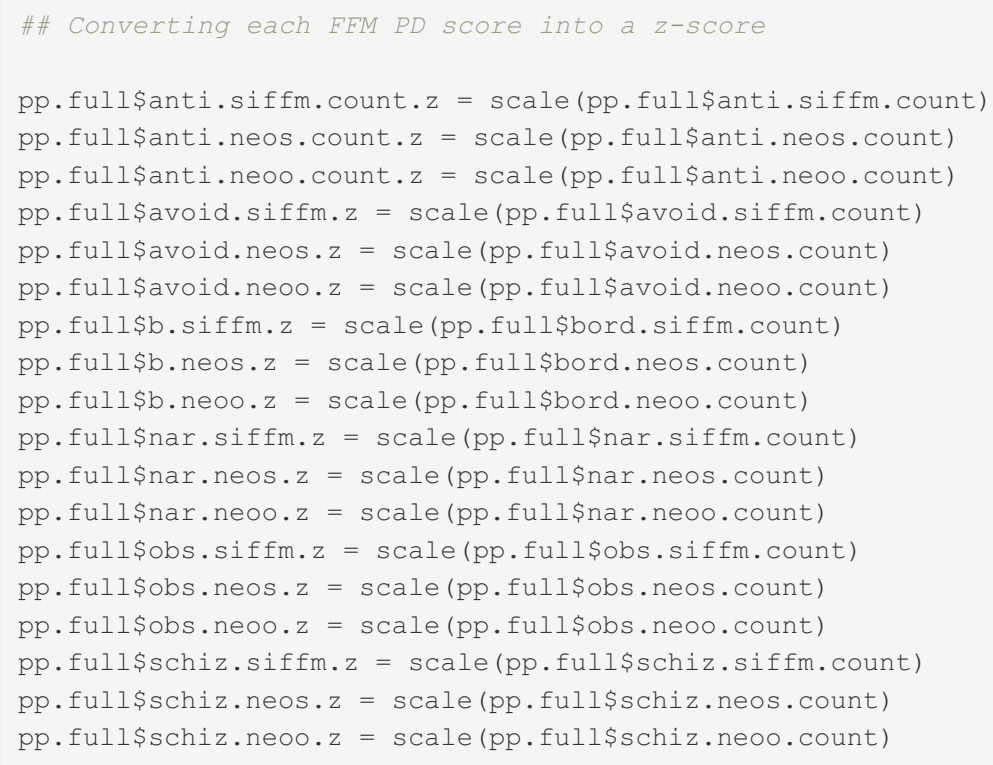

\section{Supplemental Correlation Tables}




\begin{tabular}{|c|c|c|c|c|c|c|c|c|}
\hline \#\# \# - n & & NEGAFF.S & ANTAG.S D & DISINH.S P & PSYCHOT.S & DETACH.S & NEGAFF.i & ANTAG.i \\
\hline \#\# & NEGAFF.s & 1.00 & 0.54 & 0.80 & 0.69 & 0.66 & 0.45 & 0.22 \\
\hline \#\# & ANTAG.S & 0.54 & 1.00 & 0.64 & 0.67 & 0.42 & 0.21 & 0.27 \\
\hline \#\# & DISINH.S & 0.80 & 0.64 & 1.00 & 0.71 & 0.65 & 0.41 & 0.23 \\
\hline \#\# & PSYCHOT.S & 0.69 & 0.67 & 0.71 & 1.00 & 0.59 & 0.20 & 0.14 \\
\hline \#\# \# - n & DETACH.S & 0.66 & 0.42 & 0.65 & 0.59 & 1.00 & 0.28 & 0.14 \\
\hline \#\# & NEGAFF.i & 0.45 & 0.21 & 0.41 & 0.20 & 0.28 & 1.00 & 0.61 \\
\hline \#\# & ANTAG.i & 0.22 & 0.27 & 0.23 & 0.14 & 0.14 & 0.61 & 1.00 \\
\hline \#\# \# - n & DISINH.i & 0.44 & 0.41 & 0.57 & 0.34 & 0.28 & 0.69 & 0.63 \\
\hline \#\# & PSYCHOT.i & 0.35 & 0.33 & 0.40 & 0.31 & 0.23 & 0.68 & 0.63 \\
\hline \#\# & DETACH.i & 0.29 & 0.19 & 0.33 & 0.20 & 0.38 & 0.56 & 0.47 \\
\hline \#\# & & DISINH.i & PSYCHOT.i & i DETACH.i & & & & \\
\hline \#\# & NEGAFF.S & 0.44 & 0.35 & $\begin{array}{ll}5 & 0.29\end{array}$ & & & & \\
\hline \#\# & ANTAG.S & 0.41 & 0.33 & 0.19 & & & & \\
\hline \#\# & DISINH.S & 0.57 & 0.40 & 0.33 & & & & \\
\hline \#\# & PSYCHOT.S & 0.34 & 0.31 & 0.20 & & & & \\
\hline \#\# & DETACH.S & 0.28 & 0.23 & 0.38 & & & & \\
\hline \#\# & NEGAFF.i & 0.69 & 0.68 & 0.56 & & & & \\
\hline \#\# & ANTAG.i & 0.63 & 0.63 & 0.47 & & & & \\
\hline \#\# & DISINH.i & 1.00 & 0.74 & 0.50 & & & & \\
\hline \#\# & PSYCHOT.i & 0.74 & 1.00 & 0.46 & & & & \\
\hline \#\# & DETACH.i & 0.50 & 0.46 & 1.00 & & & & \\
\hline
\end{tabular}

\#\#\#\# Table S4 (Convergent correlations between FFM PD scores)

\# Antisocial PD

anti.scores = pp.full[,c("anti.neos.count", "anti.neoo.count", "anti.siffm.count")]

round ( corr.test (anti.scores, method = "spearman")\$r), 2)

\section{\#\#}

\#\# anti.neos.count

\#\# anti.neoo.count

\#\# anti.siffm.count

anti.neos.count anti.neoo.count anti.siffm.count

$\begin{array}{lll}1.00 & 0.48 & 0.62 \\ 0.48 & 1.00 & 0.37 \\ 0.62 & 0.37 & 1.00\end{array}$

\#\# Avoidant PD

avoid.scores = pp.full[,c("avoid.neos.count", "avoid.neoo.count", "avoid.siffm.count")] round ( corr.test (avoid.scores, method = "spearman")\$r), 2)

\begin{tabular}{|c|c|c|c|}
\hline \#\# & avoid.neos. count & avoid.neoo. count & avoid.siffm.count \\
\hline \#\# avoid.neos.count & 1.00 & 0.48 & 0.72 \\
\hline \#\# avoid.neoo.count & 0.48 & 1.00 & 0.45 \\
\hline \#\# avoid.siffm.count & 0.72 & 0.45 & 1.00 \\
\hline
\end{tabular}

\#\# BPD

b.scores = pp.full[,c("bord.neos.count", "bord.neoo.count", "bord.siffm.count") ]

round ( (corr.test (b.scores, method = "spearman") \$r), 2)

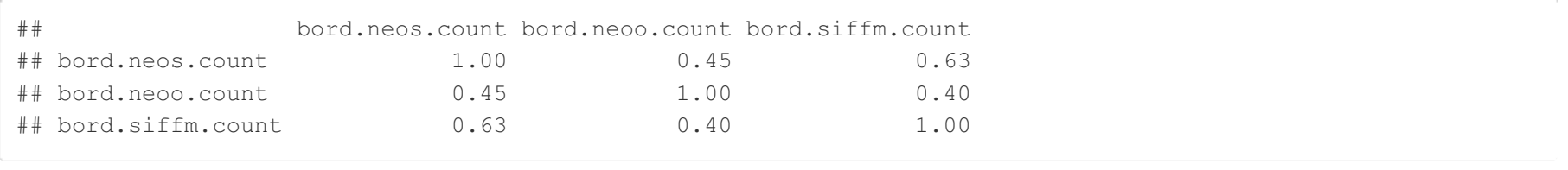

\#\# Narcissistic PD

nar.scores = pp.full[,c("nar.neos.count", "nar.neoo.count", "nar.siffm.count")]

round ( (corr.test (nar.scores, method = "spearman")\$r), 2)

\section{\#\#}

\#\# nar.neos.count

\#\# nar.neoo. count

\#\# nar.siffm.count

nar.neos.count nar.neoo.count nar.siffm.count

$\begin{array}{lll}1.00 & 0.48 & 0.62 \\ 0.48 & 1.00 & 0.34 \\ 0.62 & 0.34 & 1.00\end{array}$


obs.scores = pp.full[,c("obs.neos.count", "obs.neoo.count", "obs.siffm.count")]

round ( (corr.test (obs.scores, method = "spearman") \$r), 2)

\begin{tabular}{|lrrr|}
\hline$\# \#$ & obs.neos.count & obs.neoo.count & obs.siffm.count \\
$\# \#$ obs.neos.count & 1.00 & 0.49 & 0.65 \\
$\# \#$ obs.neoo.count & 0.49 & 1.00 & 0.46 \\
$\# \#$ obs.siffm.count & 0.65 & 0.46 & 1.00 \\
\hline
\end{tabular}

\#\# Schizotypal PD

schiz.scores = pp.full[,c("schiz.neos.count", "schiz.neoo.count", "schiz.siffm.count")]

round ( corr.test (schiz.scores, method = "spearman") \$r), 2)

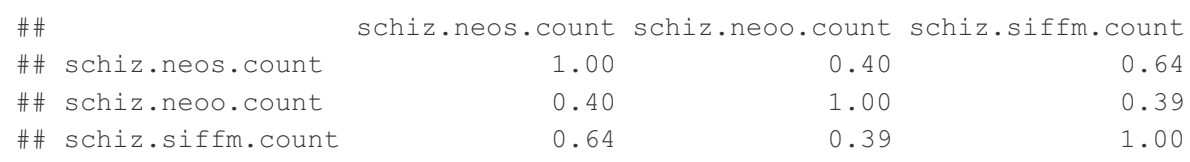

\#\#\#\# Table S5 (MTMM between PID-5-SRF and PID-5-IRF PD scores)

round ( (corr.test (pp.cor3, method = "spearman")\$r), 2)

\begin{tabular}{|c|c|c|c|c|c|c|}
\hline \#\# & & anti.pid.s & avoid.pid.s & bord.pid.s & nar.pid.s & obs.pid.s \\
\hline \#\# & anti.pid.s & 1.00 & 0.61 & 0.85 & 0.69 & 0.66 \\
\hline \#\# & avoid.pid.s & 0.61 & 1.00 & 0.79 & 0.41 & 0.83 \\
\hline \#\# & bord.pid.s & 0.85 & 0.79 & 1.00 & 0.61 & 0.75 \\
\hline \#\# & nar.pid.s & 0.69 & 0.41 & 0.61 & 1.00 & 0.59 \\
\hline \#\# & obs.pid.s & 0.66 & 0.83 & 0.75 & 0.59 & 1.00 \\
\hline \#\# & schiz.pid.s & 0.74 & 0.81 & 0.78 & 0.58 & 0.84 \\
\hline \#\# & anti.pid.i & 0.41 & 0.25 & 0.39 & 0.29 & 0.21 \\
\hline \#\# & avoid.pid.i & 0.33 & 0.43 & 0.40 & 0.12 & 0.33 \\
\hline \#\# & bord.pid.i & 0.41 & 0.36 & 0.49 & 0.25 & 0.27 \\
\hline \#\# & nar.pid.i & 0.25 & 0.14 & 0.26 & 0.29 & 0.13 \\
\hline \#\# & obs.pid.i & 0.33 & 0.32 & 0.34 & 0.21 & 0.32 \\
\hline \#\# & schiz.pid.i & 0.37 & 0.34 & 0.36 & 0.21 & 0.27 \\
\hline
\end{tabular}

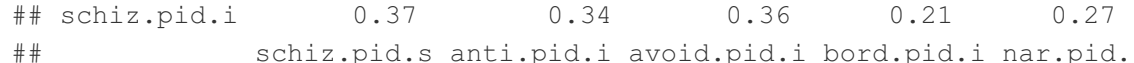

$\begin{array}{llllll}\text { \#\# anti.pid.s } & 0.74 & 0.41 & 0.33 & 0.41 & 0.25 \\ \text { \#\# avoid.pid.s } & 0.81 & 0.25 & 0.43 & 0.36 & 0.14 \\ \text { \#\# bord.pid.s } & 0.78 & 0.39 & 0.40 & 0.49 & 0.26 \\ \text { \#\# nar.pid.s } & 0.58 & 0.29 & 0.12 & 0.25 & 0.29 \\ \text { \#\# obs.pid.s } & 0.84 & 0.21 & 0.33 & 0.27 & 0.13 \\ \text { \#\# schiz.pid.s } & 1.00 & 0.25 & 0.30 & 0.29 & 0.14 \\ \text { \#\# anti.pid.i } & 0.25 & 1.00 & 0.60 & 0.86 & 0.74 \\ \text { \#\# avoid.pid.i } & 0.30 & 0.60 & 1.00 & 0.75 & 0.42 \\ \text { \#\# bord.pid.i } & 0.29 & 0.86 & 0.75 & 1.00 & 0.64 \\ \text { \#\# nar.pid.i } & 0.14 & 0.74 & 0.42 & 0.64 & 1.00 \\ \text { \#\# obs.pid.i } & 0.25 & 0.64 & 0.80 & 0.65 & 0.52 \\ \text { \#\# schiz.pid.i } & 0.30 & 0.75 & 0.82 & 0.78 & 0.60\end{array}$

\#\# obs.pid.i schiz.pid.i

\#\# anti.pid.s $\quad 0.33 \quad 0.37$

$\begin{array}{lll}\text { \#\# avoid.pid.s } & 0.32 & 0.34\end{array}$

$\begin{array}{lll}\text { \#\# bord.pid.s } & 0.34 & 0.36\end{array}$

$\begin{array}{lll}\text { \# \# nar.pid.s } & 0.21 & 0.21\end{array}$

$\begin{array}{lll}\text { \#\# obs.pid.s } & 0.32 & 0.27\end{array}$

$\begin{array}{lll}\text { \# } \text { schiz.pid.s } & 0.25 & 0.30\end{array}$

$\begin{array}{lll}\text { \# } \text { anti.pid.i } & 0.64 & 0.75\end{array}$

$\begin{array}{lll}\text { \#\# avoid.pid.i } & 0.80 & 0.82\end{array}$

$\begin{array}{lll}\text { \#\# bord.pid.i } & 0.65 & 0.78\end{array}$

$\begin{array}{lll}\text { \# n nar.pid.i } & 0.52 & 0.60\end{array}$

\#\# obs.pid.i $\quad 1.00 \quad 0.81$

$\begin{array}{lll}\# \text { \# } \text { schiz.pid.i } & 0.81 & 1.00\end{array}$ 
\#\#\#\# Table s6 (Correlations between PID-5 domains and FFM PD/GPPC scores)

pp.s5 = pp.full[,c("anti.pid.s", "anti.pid.i",

"anti.siffm.count", "anti.neos.count", "anti.neoo.count", "avoid.pid.s", "avoid.pid.i",

"avoid.siffm.count", "avoid.neos.count", "avoid.neoo.count", "bord.pid.s", "bord.pid.i"

"bord.siffm.count", "bord.neos.count", "bord.neoo.count", "nar.pid.s", "nar.pid.i",

"nar.siffm.count", "nar.neos.count", "nar.neoo.count", "obs.pid.s", "obs.pid.i",

"obs.siffm.count", "obs.neos.count", "obs.neoo.count", "schiz.pid.s", "schiz.pid.i",

"schiz.siffm.count", "schiz.neos.count", "schiz.neoo.count", "gen.z", "NEGAFF.s", "NEGA

FF.i",

TACH.i") ]

"AntAg.s", "AntAg.i", "disinh.s", "disinh.i", "psychot.s", "psychot.i", "detACH.s", "DE

\#\# Correlations with PID-5-SRF domains

round ( (corr.test (pp.s5 [, c (3, 4, 5, 8, 9, 10, 13, 14, 15, 18, 19, 20, 23, 24, 25, $28,29,30,31)], \operatorname{pp} . \mathrm{s} 5[, \mathrm{c}(32,34,36,38,40)]$,

method = "spearman") $\$ r$ ), 2)

\#\#

\#\# anti.siffm.count

\#\# anti.neos.count

\#\# anti.neoo.count

\#\# avoid.siffm.count

\#\# avoid.neos.count

\#\# avoid.neoo.count

\#\# bord.siffm.count

\#\# bord.neos.count

\#\# bord.neoo. count

\#\# nar.siffm.count

\#\# nar.neos.count

\#\# nar.neoo.count

\#\# obs.siffm.count

\#\# obs.neos.count

\#\# obs.neoo.count

\#\# schiz.siffm.count

\#\# schiz.neos.count

\#\# schiz.neoo.count

\#\# gen.z
NEGAFF.S ANTAG.S DISINH.S PSYCHOT.S DETACH.S

$\begin{array}{rrrrr}-0.03 & 0.31 & 0.08 & 0.10 & -0.10 \\ 0.14 & 0.50 & 0.27 & 0.31 & 0.09 \\ 0.07 & 0.25 & 0.11 & 0.16 & 0.05 \\ 0.31 & 0.02 & 0.25 & 0.13 & 0.46 \\ 0.49 & 0.03 & 0.44 & 0.25 & 0.65 \\ 0.24 & -0.02 & 0.24 & 0.04 & 0.35 \\ 0.55 & 0.30 & 0.51 & 0.37 & 0.38 \\ 0.70 & 0.31 & 0.59 & 0.47 & 0.47 \\ 0.37 & 0.04 & 0.32 & 0.14 & 0.25 \\ -0.21 & 0.23 & -0.12 & 0.00 & -0.11 \\ 0.14 & 0.53 & 0.26 & 0.32 & 0.15 \\ 0.06 & 0.25 & 0.08 & 0.15 & 0.11 \\ -0.18 & -0.19 & -0.33 & -0.20 & -0.04 \\ -0.25 & -0.26 & -0.49 & -0.32 & -0.18 \\ -0.22 & -0.28 & -0.41 & -0.29 & -0.08 \\ 0.29 & 0.13 & 0.31 & 0.23 & 0.52 \\ 0.50 & 0.24 & 0.54 & 0.44 & 0.66 \\ 0.25 & 0.09 & 0.27 & 0.16 & 0.40 \\ 0.44 & 0.32 & 0.40 & 0.34 & 0.56\end{array}$

\#\# Correlations with PID-5-IRF domains

round ( (corr.test (pp.s5 [, c (3, 4, 5, 8, 9, 10, 13, 14, 15, 18, 19, 20, 23, 24, 25, $28,29,30,31)], \operatorname{pp} . \mathrm{s} 5[, \mathrm{c}(33,35,37,39,41)]$,

method = "spearman") $\$ r$ ), 2)

\#\#

\#\# anti.siffm.count

\#\# anti.neos.count

\#\# anti.neoo.count

\#\# avoid.siffm.count

\#\# avoid.neos.count

\#\# avoid.neoo.count

\#\# bord.siffm.count

\#\# bord.neos.count

\#\# bord.neoo.count

\#\# nar.siffm.count

\#\# nar.neos.count

\#\# nar.neoo.count

\#\# obs.siffm.count

\#\# obs.neos.count

\#\# obs.neoo.count

\#\# schiz.siffm.count

\#\# schiz.neos.count

\#\# schiz.neoo. count

\#\# gen.z
NEGAFF. i ANTAG.i DISINH.i PSYCHOT.i DETACH.i

$\begin{array}{rrrrr}0.05 & 0.21 & 0.26 & 0.22 & -0.05 \\ 0.18 & 0.35 & 0.36 & 0.33 & 0.14 \\ 0.33 & 0.68 & 0.47 & 0.41 & 0.20 \\ 0.24 & -0.01 & 0.05 & 0.05 & 0.32 \\ 0.28 & 0.03 & 0.16 & 0.14 & 0.34 \\ 0.38 & -0.04 & 0.13 & 0.09 & 0.56 \\ 0.44 & 0.31 & 0.42 & 0.36 & 0.20 \\ 0.44 & 0.25 & 0.42 & 0.35 & 0.31 \\ 0.75 & 0.43 & 0.49 & 0.41 & 0.39 \\ -0.08 & 0.14 & 0.09 & 0.09 & -0.04 \\ 0.16 & 0.33 & 0.35 & 0.30 & 0.16 \\ 0.31 & 0.70 & 0.40 & 0.38 & 0.30 \\ -0.10 & -0.07 & -0.32 & -0.17 & 0.02 \\ -0.19 & -0.06 & -0.36 & -0.22 & -0.11 \\ -0.35 & -0.32 & -0.71 & -0.45 & -0.11 \\ 0.21 & 0.08 & 0.17 & 0.14 & 0.35 \\ 0.28 & 0.06 & 0.29 & 0.26 & 0.34 \\ 0.46 & 0.19 & 0.31 & 0.29 & 0.65 \\ 0.54 & 0.45 & 0.41 & 0.40 & 0.55\end{array}$


pp.s6 = pp.full[, c("NEGAFF.s", "NEGAFF.i",

"ANTAG.s", "ANTAg.i", "Disinh.s", "DISINH.i", "PSYCHOT.s", "PSYCHOT.i", "DETACH.s", "D ETACH.i",

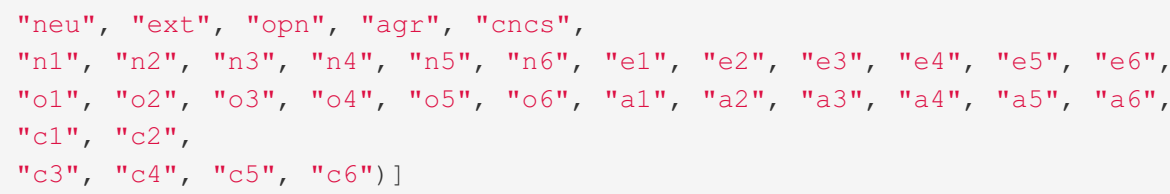

\begin{tabular}{|c|c|c|c|c|c|c|}
\hline \#\# & & NEGAFF.S & ANTAG.S & DISINH.S & PSYCHOT.S & DETACH.S \\
\hline \#\# & neu & 0.63 & 0.31 & 0.58 & 0.40 & 0.51 \\
\hline \#\# & ext & -0.08 & 0.05 & -0.08 & -0.04 & -0.41 \\
\hline \#\# & opn & 0.11 & 0.07 & 0.10 & 0.20 & -0.01 \\
\hline \#\# & $\operatorname{agr}$ & 0.13 & -0.25 & 0.05 & -0.03 & -0.03 \\
\hline \#\# & encs & -0.22 & -0.17 & -0.37 & -0.15 & -0.18 \\
\hline \#\# & $\mathrm{n} 1$ & 0.60 & 0.25 & 0.50 & 0.32 & 0.43 \\
\hline \#\# & $\mathrm{n} 2$ & 0.41 & 0.29 & 0.31 & 0.30 & 0.32 \\
\hline \#\# & n3 & 0.48 & 0.15 & 0.40 & 0.30 & 0.43 \\
\hline \#\# & $\mathrm{n} 4$ & 0.39 & 0.18 & 0.38 & 0.25 & 0.40 \\
\hline \#\# & n5 & 0.43 & 0.32 & 0.50 & 0.32 & 0.28 \\
\hline \#\# & $\mathrm{n} 6$ & 0.52 & 0.18 & 0.44 & 0.26 & 0.41 \\
\hline \#\# & e1 & 0.11 & 0.04 & 0.05 & 0.05 & -0.26 \\
\hline \#\# & e2 & -0.08 & -0.08 & -0.12 & -0.15 & -0.33 \\
\hline \#\# & e3 & -0.12 & 0.02 & -0.17 & -0.07 & -0.21 \\
\hline \#\# & e4 & -0.18 & -0.06 & -0.17 & -0.06 & -0.38 \\
\hline \#\# & e 5 & 0.08 & 0.21 & 0.13 & 0.12 & -0.22 \\
\hline \#\# & e 6 & -0.02 & -0.02 & -0.01 & -0.01 & -0.24 \\
\hline \#\# & $\circ 1$ & 0.36 & 0.32 & 0.36 & 0.37 & 0.20 \\
\hline \#\# & $\circ 2$ & -0.08 & -0.07 & -0.05 & 0.00 & -0.15 \\
\hline \#\# & ०3 & 0.42 & 0.07 & 0.32 & 0.23 & 0.18 \\
\hline \#\# & $\circ 4$ & -0.14 & -0.01 & -0.08 & -0.02 & -0.19 \\
\hline \#\# & ०5 & 0.04 & 0.06 & 0.00 & 0.19 & 0.11 \\
\hline \#\# & 06 & -0.09 & -0.05 & -0.08 & 0.01 & -0.13 \\
\hline \#\# & a1 & -0.14 & -0.29 & -0.17 & -0.21 & -0.27 \\
\hline \#\# & a2 & -0.01 & -0.24 & -0.13 & -0.05 & -0.05 \\
\hline \#\# & a3 & 0.01 & -0.21 & -0.08 & -0.05 & -0.08 \\
\hline \#\# & a 4 & 0.09 & -0.09 & 0.15 & 0.02 & 0.04 \\
\hline \#\# & a5 & 0.21 & -0.13 & 0.18 & 0.01 & 0.24 \\
\hline \#\# & a 6 & 0.25 & -0.02 & 0.17 & 0.16 & 0.03 \\
\hline \#\# & c1 & -0.18 & -0.15 & -0.32 & -0.13 & -0.12 \\
\hline \#\# & $c 2$ & 0.00 & 0.06 & -0.08 & 0.00 & -0.02 \\
\hline \#\# & c3 & -0.13 & -0.13 & -0.22 & -0.08 & -0.09 \\
\hline \#\# & C4 & -0.11 & -0.07 & -0.16 & -0.04 & -0.09 \\
\hline \#\# & c5 & -0.32 & -0.22 & -0.40 & -0.26 & -0.27 \\
\hline \#\# & c6 & -0.21 & -0.18 & -0.29 & -0.15 & -0.08 \\
\hline
\end{tabular}

\#\# Correlations with PID-5-IRF domains

round ( (corr.test (pp.s6[,c(11:45)], pp.s6[,c(2,4,6, 8, 10) ], method = "spearman") \$r), 2) 


\begin{tabular}{|c|c|c|c|c|c|c|}
\hline \#\# & & NEGAFF.i & ANTAG.i & DISINH.i & PSYCHOT.i & DETACH.i \\
\hline \#\# & neu & 0.47 & 0.31 & 0.42 & 0.37 & 0.32 \\
\hline \#\# & ext & -0.12 & 0.09 & 0.06 & 0.05 & -0.29 \\
\hline \#\# & opn & -0.06 & 0.04 & 0.09 & 0.10 & -0.14 \\
\hline \#\# & $\operatorname{agr}$ & -0.01 & -0.10 & -0.07 & -0.09 & -0.10 \\
\hline \#\# & encs & -0.19 & -0.03 & -0.31 & -0.15 & -0.17 \\
\hline \#\# & n1 & 0.43 & 0.30 & 0.38 & 0.35 & 0.29 \\
\hline \#\# & $\mathrm{n} 2$ & 0.29 & 0.21 & 0.28 & 0.21 & 0.20 \\
\hline \#\# & n3 & 0.40 & 0.20 & 0.28 & 0.27 & 0.26 \\
\hline \#\# & $\mathrm{n} 4$ & 0.30 & 0.19 & 0.26 & 0.21 & 0.28 \\
\hline \#\# & n5 & 0.34 & 0.28 & 0.44 & 0.35 & 0.09 \\
\hline \#\# & n6 & 0.38 & 0.20 & 0.32 & 0.23 & 0.23 \\
\hline \#\# & e1 & 0.01 & 0.08 & 0.07 & 0.05 & -0.22 \\
\hline \#\# & e2 & -0.11 & -0.03 & -0.01 & 0.00 & -0.21 \\
\hline \#\# & e3 & -0.16 & 0.06 & 0.00 & -0.08 & -0.18 \\
\hline \#\# & e4 & -0.12 & 0.04 & -0.04 & 0.05 & -0.22 \\
\hline \#\# & e5 & 0.07 & 0.20 & 0.26 & 0.23 & -0.07 \\
\hline \#\# & e6 & -0.12 & 0.00 & 0.01 & -0.09 & -0.31 \\
\hline \#\# & o1 & 0.15 & 0.16 & 0.28 & 0.25 & 0.09 \\
\hline \#\# & o2 & -0.05 & -0.02 & -0.02 & 0.04 & -0.20 \\
\hline \#\# & 03 & 0.24 & 0.10 & 0.15 & 0.11 & 0.11 \\
\hline \#\# & $\circ 4$ & -0.12 & 0.07 & 0.03 & 0.11 & -0.18 \\
\hline \#\# & 05 & -0.20 & -0.07 & -0.06 & -0.06 & -0.16 \\
\hline \#\# & 06 & -0.20 & -0.11 & -0.06 & -0.11 & -0.18 \\
\hline \#\# & a1 & -0.10 & -0.15 & -0.12 & -0.10 & -0.16 \\
\hline \#\# & $\mathrm{a} 2$ & -0.07 & -0.08 & -0.18 & -0.11 & -0.13 \\
\hline \#\# & a3 & -0.04 & -0.03 & -0.08 & -0.06 & -0.12 \\
\hline \#\# & a 4 & 0.00 & -0.14 & -0.02 & -0.10 & 0.00 \\
\hline \#\# & a5 & 0.07 & -0.09 & 0.03 & 0.00 & 0.09 \\
\hline \#\# & a 6 & 0.10 & 0.03 & 0.05 & 0.01 & 0.01 \\
\hline \#\# & $\mathrm{c} 1$ & -0.21 & -0.14 & -0.28 & -0.19 & -0.22 \\
\hline \#\# & c2 & -0.02 & 0.11 & -0.08 & 0.00 & -0.09 \\
\hline \#\# & c3 & -0.06 & 0.00 & -0.22 & -0.12 & 0.00 \\
\hline \#\# & $\mathrm{C} 4$ & -0.07 & 0.05 & -0.13 & -0.04 & -0.09 \\
\hline \#\# & c5 & -0.26 & -0.08 & -0.33 & -0.23 & -0.15 \\
\hline \#\# & c6 & -0.21 & -0.13 & -0.27 & -0.12 & -0.04 \\
\hline
\end{tabular}

\#\#\#\# Table S8 (Correlations between PID-5 PD scores and FFM PD/GPPC scores)

\#\# Correlations with PID-5-SRF scores

round((corr.test (pp.s5[,c(3, 4, 5, 8, 9, 10, 13, 14, 15, 18, 19, 20, 23, 24, 25, 28, 29, 30, 31)], pp.s5[,c(1, 6, 11, 16, 21, 26)],

method = "spearman") $\$ r), 2$ ) 
\#\# anti.siffm.count

\#\# anti.neos.count

\#\# anti.neoo.count

\#\# avoid.siffm.count

\#\# avoid.neos.count

\#\# avoid.neoo.count

\#\# bord.siffm.count

\#\# bord.neos.count

\#\# bord.neoo.count

\#\# nar.siffm.count

\#\# nar.neos.count

\#\# nar.neoo.count

\#\# obs.siffm.count

\#\# obs.neos.count

\#\# obs.neoo.count

\#\# schiz.siffm.count

\#\# schiz.neos.count

\#\# schiz.neoo.count

\#\# gen.z

\#\#

\#\# anti.siffm.count

\#\# anti.neos.count

\#\# anti.neoo.count

\#\# avoid.siffm.count

\#\# avoid.neos.count

\#\# avoid.neoo.count

\#\# bord.siffm.count

\#\# bord.neos.count

\#\# bord.neoo.count

\#\# nar.siffm.count

\#\# nar.neos.count

\#\# nar.neoo.count

\#\# obs.siffm.count

\#\# obs.neos.count

\#\# obs.neoo.count

\#\# schiz.siffm.count

\#\# schiz.neos.count

\#\# schiz.neoo.count

\#\# gen.z anti.pid.s avoid.pid.s bord.pid.s nar.pid.s obs.pid.s

$\begin{array}{rrrrr}0.26 & -0.10 & 0.07 & 0.35 & -0.07 \\ 0.49 & 0.11 & 0.27 & 0.43 & 0.13 \\ 0.25 & 0.08 & 0.15 & 0.22 & 0.05 \\ 0.13 & 0.48 & 0.28 & -0.10 & 0.32 \\ 0.21 & 0.68 & 0.45 & -0.04 & 0.46 \\ 0.09 & 0.36 & 0.22 & -0.08 & 0.27 \\ 0.47 & 0.46 & 0.59 & 0.30 & 0.32 \\ 0.52 & 0.60 & 0.71 & 0.26 & 0.42 \\ 0.18 & 0.32 & 0.36 & 0.02 & 0.19 \\ 0.12 & -0.14 & -0.10 & 0.23 & -0.08 \\ 0.51 & 0.16 & 0.28 & 0.42 & 0.18 \\ 0.23 & 0.12 & 0.14 & 0.22 & 0.10 \\ -0.30 & -0.06 & -0.27 & -0.18 & 0.05 \\ -0.42 & -0.21 & -0.35 & -0.17 & -0.10 \\ -0.36 & -0.11 & -0.30 & -0.20 & -0.08 \\ 0.26 & 0.52 & 0.34 & 0.03 & 0.31 \\ 0.41 & 0.68 & 0.54 & 0.10 & 0.49 \\ 0.18 & 0.40 & 0.26 & -0.04 & 0.26 \\ 0.42 & 0.60 & 0.50 & 0.22 & 0.44\end{array}$

schiz.pid.s

0.00

0.26

0.14

0.30

0.43

0.20

0.37

0.48

0.19

$-0.04$

0.30

0.16

$-0.10$

$-0.26$

$-0.24$

0.40

0.56

0.29

0.48

\#\# Correlations with PID-5-IRF scores

round ( (corr.test (pp.s5 [, c (3, 4, 5, 8, 9, 10, 13, 14, 15, 18, 19, 20, 23, 24, 25, 28, 29, 30, 31) ], pp.s5[,c (2, 7, 12, 17, 22, 27)], method = "spearman") \$r), 2) 
\#\# anti.siffm.count

\#\# anti.neos.count

\#\# anti.neoo.count

\#\# avoid.siffm.count

\#\# avoid.neos.count

\#\# avoid.neoo.count

\#\# bord.siffm.count

\#\# bord.neos.count

\#\# bord.neoo.count

\#\# nar.siffm.count

\#\# nar.neos.count

\#\# nar.neoo.count

\#\# obs.siffm.count

\#\# obs.neos.count

\#\# obs.neoo.count

\#\# schiz.siffm.count

\#\# schiz.neos.count

\#\# schiz.neoo.count

\#\# gen.z

\#\#

\#\# anti.siffm.count

\#\# anti.neos.count

\#\# anti.neoo.count

\#\# avoid.siffm.count

\#\# avoid.neos.count

\#\# avoid.neoo.count

\#\# bord.siffm. count

\#\# bord.neos.count

\#\# bord.neoo.count

\#\# nar.siffm.count

\#\# nar.neos.count

\#\# nar.neoo.count

\#\# obs.siffm.count

\#\# obs.neos.count

\#\# obs.neoo.count

\#\# schiz.siffm.count

\#\# schiz.neos.count

\#\# schiz.neoo.count

\#\# gen.z anti.pid.i avoid.pid.i bord.pid.i nar.pid.i obs.pid.i
0.28
$-0.02$
0.16
0.22
0.13

0.42

0.17

0.29

0.33

0.24

0.70

0.24

0.49

0.16

0.62

0.31

$-0.02$

0.34

0.23

$-0.04$

0.15

0.07

0.36

0.28

0.03

0.57

0.47

$-0.06$

0.22

0.39

0.39

$-0.16$

0.32

0.34

0.46

0.27

0.27

0.52

0.17

0.54

0.73

0.01

0.27

0.46

0.68

0.18

0.23

0.29

0.68
-0.20

0.31

0.46
-0.18

0.35

0.29

0.15

0.11

0.30

0.27

$-0.21$

$-0.52$

$-0.25$

$-0.48$

$-0.16$

0.62

0.37

$-0.05$

$-0.07$

$-0.07$

$-0.32$

$-0.13$

0.12

0.34

0.20

0.28

0.43

0.01

$-0.18$

0.17

0.28

0.49

0.67

0.61

0.56

$-0.01$

0.20

0.09

0.23

schiz.pid.i

0.14

0.31

0.43

0.19

0.22

0.28

0.31

0.31

0.42

0.09

0.32

0.47

$-0.08$

$-0.16$

$-0.28$

0.25

0.29

0.49

0.55

\section{Main Analyses}

\section{Self-other agreement for PID-5 Domains}

\#\# Negative Affect

\# Mean-level bias

t.test (pp.full\$NEGAFF.s, pp.full\$NEGAFF.i, paired $=T$ )

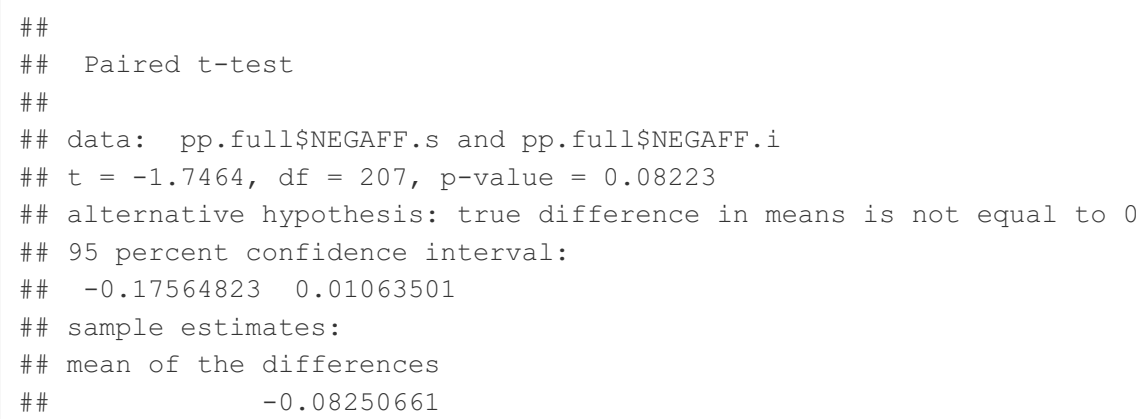


\#\# Wilcoxon signed rank test with continuity correction

\#\# data: pp.full\$NEGAFF.s and pp.full\$NEGAFF.i

\#\# $\mathrm{V}=9572, \mathrm{p}$-value $=0.1673$

\#\# alternative hypothesis: true location shift is not equal to 0

\# Cohen's dz

(1.7464/sqrt (208))

\# \# [1] 0.1210911

\# Cohen's U3

$\operatorname{pnorm}(.121)$

\#\# [1] 0.5481545

\# OVL

$(2 * \operatorname{pnorm}((-\operatorname{abs}(.121)) / 2))$

\#\# [1] 0.9517574

\# Correlational accuracy

print(corr.test (pp.full\$NEGAFF.s, pp.full\$NEGAFF.i, method = "spearman"), short = F, digits = 3)

\#\# Call:corr.test ( $x=$ pp.full\$NEGAFF.s, $y=$ pp.full\$NEGAFF.i, method = "spearman")

\#\# Correlation matrix

\# \# [1] 0.447

\#\# Sample Size

\#\# [1] 208

\#\# Probability values adjusted for multiple tests.

\#\# [1] 0

\#\#

\#\# Confidence intervals based upon normal theory. To get bootstrapped values, try cor.ci

\#\# raw.lower raw.r raw.upper raw.p lower.adj upper.adj

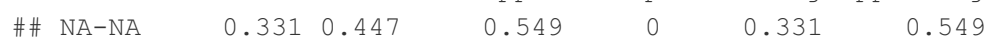

\section{\#\# Antagonism}

\# Mean-level bias

t.test (pp.full\$ANTAG.s, pp.full\$ANTAG.i, paired = T)

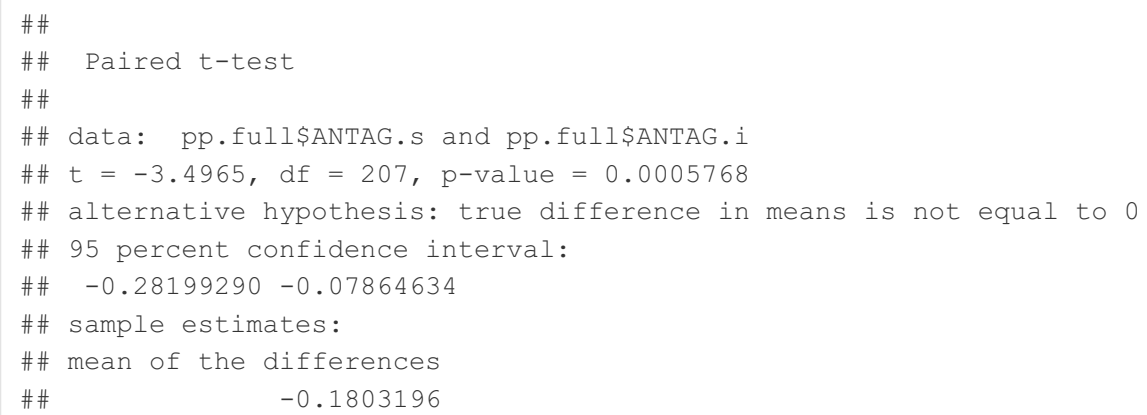

wilcox.test (pp.full\$ANTAG.s, pp.full\$ANTAG.i, paired $=\mathrm{T}$ )

\#\# $V=8358, p$-value $=0.007203$

\#\# alternative hypothesis: true location shift is not equal to 0 
\# [1] 0.2424387

pnorm (.242)

\#\# [1] 0.5956099

$(2 * \operatorname{pnorm}((-\mathrm{abs}(.242)) / 2))$

\#\# [1] 0.903691

\# Corelational accuracy

print(corr.test (pp.full\$ANTAG.s, pp.full\$ANTAG.i, method = "spearman"), short = F, digits = 3)

\#\# Call:corr.test ( $\mathrm{x}=$ pp.full\$ANTAG.s, $y=$ pp.full\$ANTAG.i, method = "spearman")

\#\# Correlation matrix

\#\# [1] 0.268

\#\# Sample Size

\#\# [1] 208

\#\# Probability values adjusted for multiple tests.

\#\# [1] 0

\#\#

\#\# Confidence intervals based upon normal theory. To get bootstrapped values, try cor.ci

\#\# raw.lower raw.r raw.upper raw.p lower.adj upper.adj

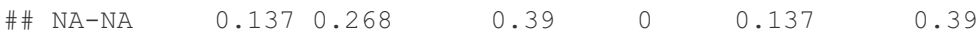

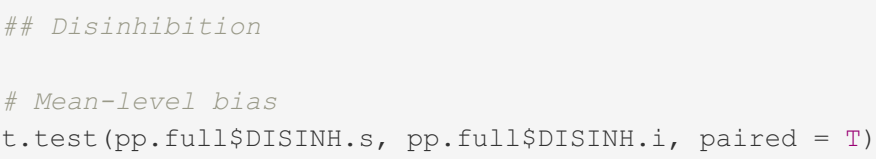

\#\# Disinhibition

\# Mean-level bias

t.test(pp.full\$DISINH.s, pp.full\$DISINH.i, paired $=\mathrm{T}$ )

\#\#

\#\# Paired t-test

\#\# data: pp.full\$DISINH.s and pp.full\$DISINH.i

$\#$ \#\# $t=-6.0314, \mathrm{df}=207, \mathrm{p}$-value $=7.377 \mathrm{e}-09$

\#\# alternative hypothesis: true difference in means is not equal to 0

\#\# 95 percent confidence interval:

\#\# $-0.3117628-0.1581592$

\#\# sample estimates:

\#\# mean of the differences

\#\#

$-0.234961$

wilcox.test (pp.full\$DISINH.s, pp.full\$DISINH.i, paired $=\mathrm{T}$ )

\#\#

\#\# Wilcoxon signed rank test with continuity correction

\#\#

\#\# data: pp.full\$DISINH.s and pp.full\$DISINH.i

\#\# $\mathrm{V}=6138, \mathrm{p}$-value $=8.289 \mathrm{e}-08$

\#\# alternative hypothesis: true location shift is not equal to 0

$(6.0314 / \operatorname{sqrt}(208))$

\#\# [1] 0.4182023

$\operatorname{pnorm}(.418)$

\#\# [1] 0.6620264 
\# Correlational accuracy

print(corr.test(pp.full\$DISINH.s, pp.full\$DISINH.i, method = "spearman"), short = F, digits = 3)

\#\# Call:corr.test $(\mathrm{x}=\mathrm{pp}$.full\$DISINH.s, $\mathrm{y}=$ pp.full\$DISINH.i, method = "spearman")

\#\# Correlation matrix

\#\# [1] 0.566

\#\# Sample Size

\#\# [1] 208

\#\# Probability values adjusted for multiple tests.

\#\# [1] 0

\#\#

\# Confidence intervals based upon normal theory. To get bootstrapped values, try cor.ci

\#\# raw.lower raw.r raw.upper raw.p lower.adj upper.adj

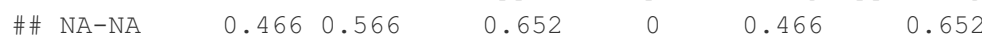

\section{\#\# Psychoticism}

\# Mean-level bias

t.test (pp.full\$PSYCHOT.s, pp.full\$PSYCHOT.i, paired = T)

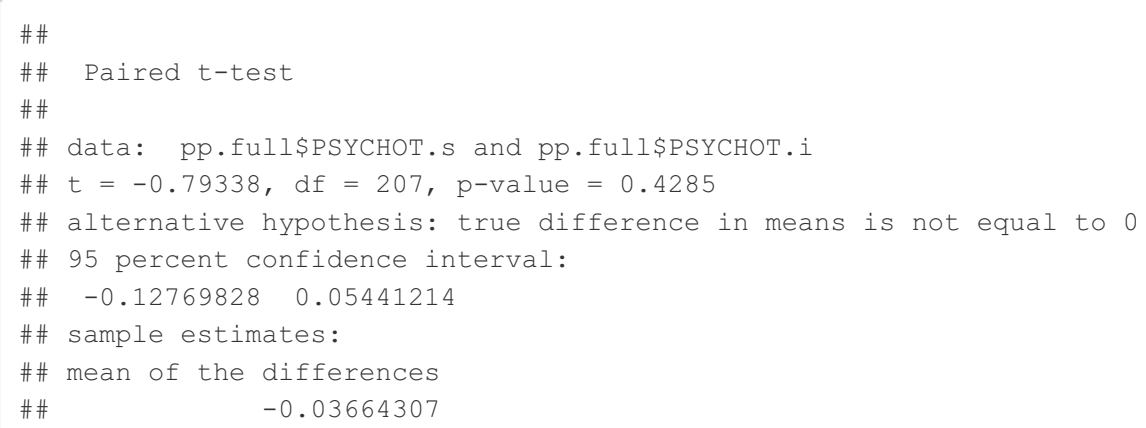

wilcox.test (pp.full\$PSYCHOT.s, pp.full\$PSYCHOT.i, paired $=\mathrm{T}$ )

\#\# $\mathrm{V}=9317.5, \mathrm{p}$-value $=0.5095$

\#\# alternative hypothesis: true location shift is not equal to 0

$(.79338 / \operatorname{sqrt}(208))$

\#\# [1] 0.05501101

pnorm (.055)

\#\# [1] 0.5219308

$(2 * \operatorname{pnorm}((-\operatorname{abs}(.055)) / 2))$

\#\# [1] 0.9780609

\# Correlational accuracy

print (corr.test (pp.full\$PSYCHOT.s, pp.full\$PSYCHOT.i, method = "spearman"), short = F, digits = 3) 
\#\# Call:corr.test $(x=$ pp.full\$PSYCHOT.s, $y=$ pp.full\$PSYCHOT.i, method = "spearman")

\#\# Correlation matrix

\#\# [1] 0.31

\#\# Sample Size

\#\# [1] 208

\#\# Probability values adjusted for multiple tests.

\#\# [1] 0

\#\#

\#\# Confidence intervals based upon normal theory. To get bootstrapped values, try cor.ci

\#\# raw.lower raw.r raw.upper raw.p lower.adj upper.adj

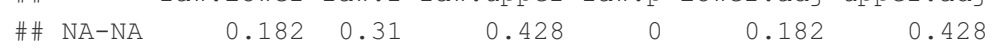

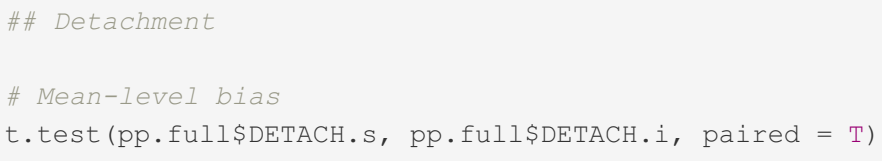

(1.6062/sqrt (208))

\#\# [1] 0.1113699

pnorm (.111)

\#\# [1] 0.5441918

$(2 * \operatorname{pnorm}((-\operatorname{abs}(.111)) / 2))$

\#\# [1] 0.9557401

* Correlational accuracy

print(corr.test(pp.full\$DETACH.s, pp.full\$PSYCHOT.i, method = "spearman"), short = F, digits = 3) 
\#\# Call:corr.test $(x=$ pp.full\$DETACH.s, $y=$ pp.full\$PSYCHOT.i, method = "spearman")

\#\# Correlation matrix

\#\# [1] 0.235

\#\# Sample Size

\#\# [1] 208

\#\# Probability values adjusted for multiple tests.

\#\# [1] 0.001

\#\#

\# Confidence intervals based upon normal theory. To get bootstrapped values, try cor.ci

\#\# raw.lower raw.r raw.upper raw.p lower.adj upper.adj
\#\# NA-NA
$0.102 \quad 0.235$
0.3590 .001
0.102
0.359

\#\#\#\#\# Regression models \#\#\#\#\#\#

\#\#\#\# Negative Affect

\#\# Centering \#\#

pp.full\$negaff.s_c $=$ pp.full\$NEGAFF.s - mean (pp.full\$NEGAFF.s)

pp.full\$negaff.i_c $=$ pp.full\$NEGAFF.i - mean(pp.full\$NEGAFF.s)

\#\# Base model

negaff.model1 = lm(negaff.i_c 1 + negaff.s_c, data $=$ pp.full)

summary (negaff.model1)

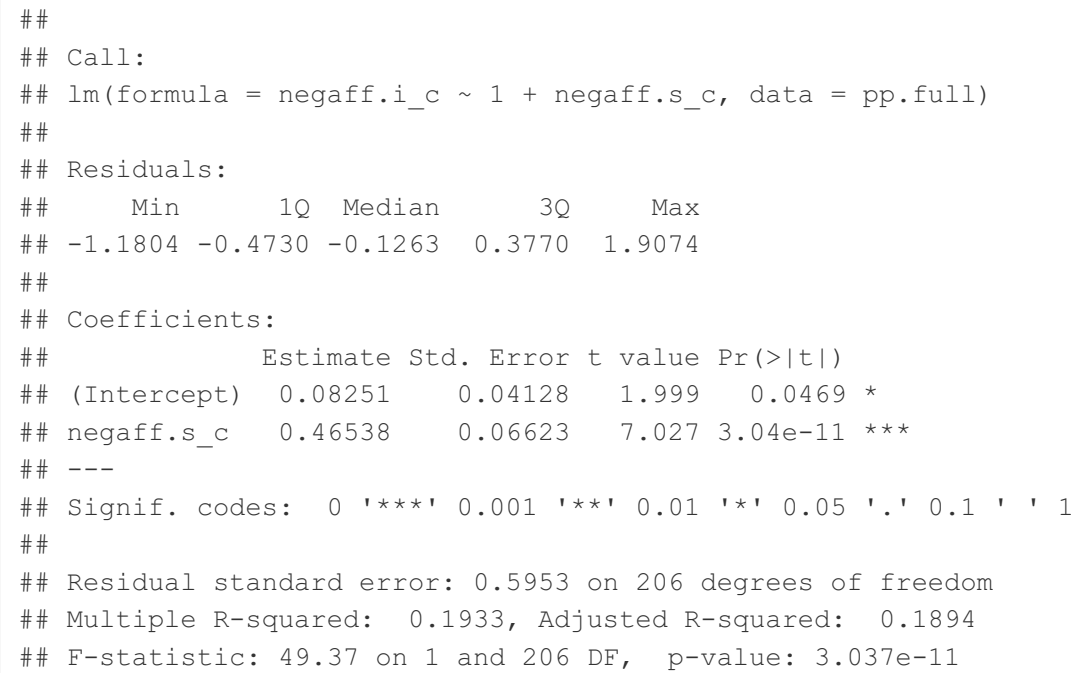

round (confint (negaff.model1), 3)

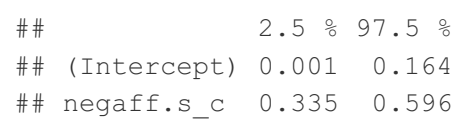


\#\# Call:

\#\# $\operatorname{lm}$ (formula $=$ negaff.i $c \sim$ negaff.s $c *$ gen.z, data $=$ pp.full)

\#\#

\#\# Residuals:

\#\# Min 1Q Median 3Q Max

$\begin{array}{llllll}\# \# & -1.0941 & -0.3941 & -0.0764 & 0.3376 & 1.7213\end{array}$

\#\#

\#\# Coefficients:

\#\# Estimate std. Error $t$ value Pr $(>|t|)$

$\begin{array}{lllllll}\text { \#\# (Intercept) } \quad 0.081288 & 0.041594 & 1.954 & 0.052032\end{array}$.

$\begin{array}{lllll}\text { \#\# negaff.s_c } & 0.262363 & 0.069417 & 3.780 & 0.000206\end{array}$ ***

\#\# gen.z $\quad 0.278970 \quad 0.042420 \quad 6.576 \quad 3.97 e-10 * \star *$

$\begin{array}{llllll}\text { \#\# negaff.s_c:gen.z } & 0.004336 & 0.062860 & 0.069 & 0.945073\end{array}$

\#\#---

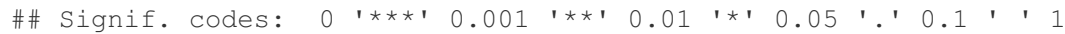

\#\#

\#\# Residual standard error: 0.5431 on 204 degrees of freedom

\#\# Multiple R-squared: 0.3352, Adjusted R-squared: 0.3254

\#\# F-statistic: 34.28 on 3 and 204 DF, p-value: $<2.2 e-16$

round (confint (negaff.model2), 3)

\begin{tabular}{|rrr}
\hline$\# \#$ & $2.5 \%$ & $97.5 \%$ \\
$\# \#$ (Intercept) & -0.001 & 0.163 \\
$\# \#$ negaff.s_c & 0.125 & 0.399 \\
$\# \#$ gen.z & 0.195 & 0.363 \\
\#\# negaff.s_c:gen.z & -0.120 & 0.128
\end{tabular}

\# R2 increase significance

anova (negaff.model1, negaff.model2)

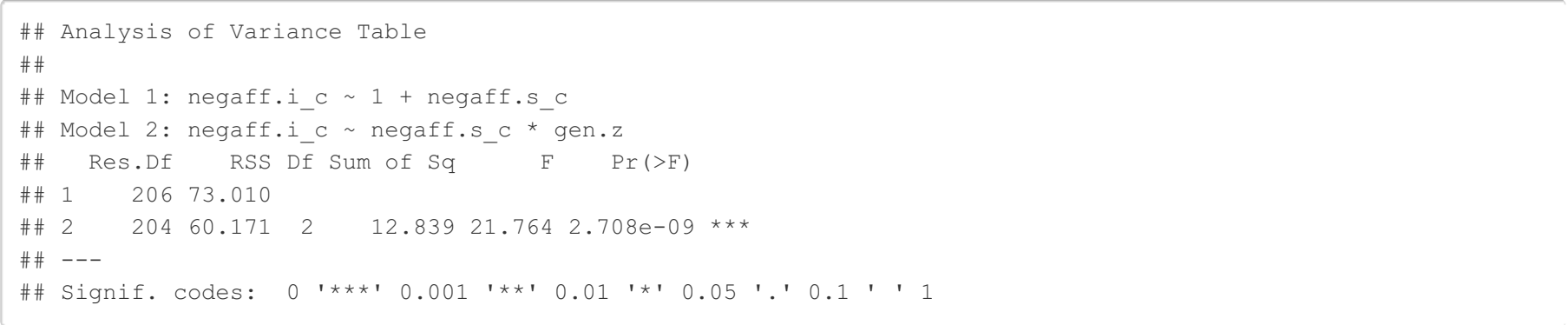

\#\# Test of simple intercepts

at. negaffiactor $=\operatorname{seq}(-2.69,3.01, .01)$

intercepts.negaff $=$ negaff.model2\$coef [1] + negaff.model2\$coef[3]*at.negafffactor

estmean.negaff $=\operatorname{coef}($ negaff.model2)

var.negaff $=\operatorname{vcov}($ negaff.model2)

se.negaff $=\operatorname{rep}(\mathrm{NA}$, length (at.negafffactor))

for( $i$ in $1:$ length (at.negafffactor)) \{

$j=$ at.negafffactor [i]

se.negaff $[i]=$ deltamethod $(\sim(x 1)+(x 3) * j$, estmean.negaff, var.negaff $)$

\}

upper.negaff $=$ intercepts.negaff $+1.96 *$ se.negaff

lower.negaff $=$ intercepts.negaff $-1.96 *$ se.negaff

negaff.intercepts = cbind(at.negafffactor, intercepts.negaff, lower.negaff, upper.negaff)

negaff.intercepts = as.data.frame(negaff.intercepts) \#\# Dataframe gives CIS for intercepts at different \#\# values of the GPPC 
\#\# Centering \#\#

pp.full\$ant.s_c $=$ pp.full\$ANTAG.s - mean (pp.full\$ANTAG.s)

pp.full\$ant.i_c $=$ pp.full\$ANTAG.i - mean (pp.full\$ANTAG.s)

\#\# Base model

ant.modell $=\operatorname{lm}($ ant.i $c \sim 1+$ ant.s_c, data $=$ pp.full)

summary (ant.model1)

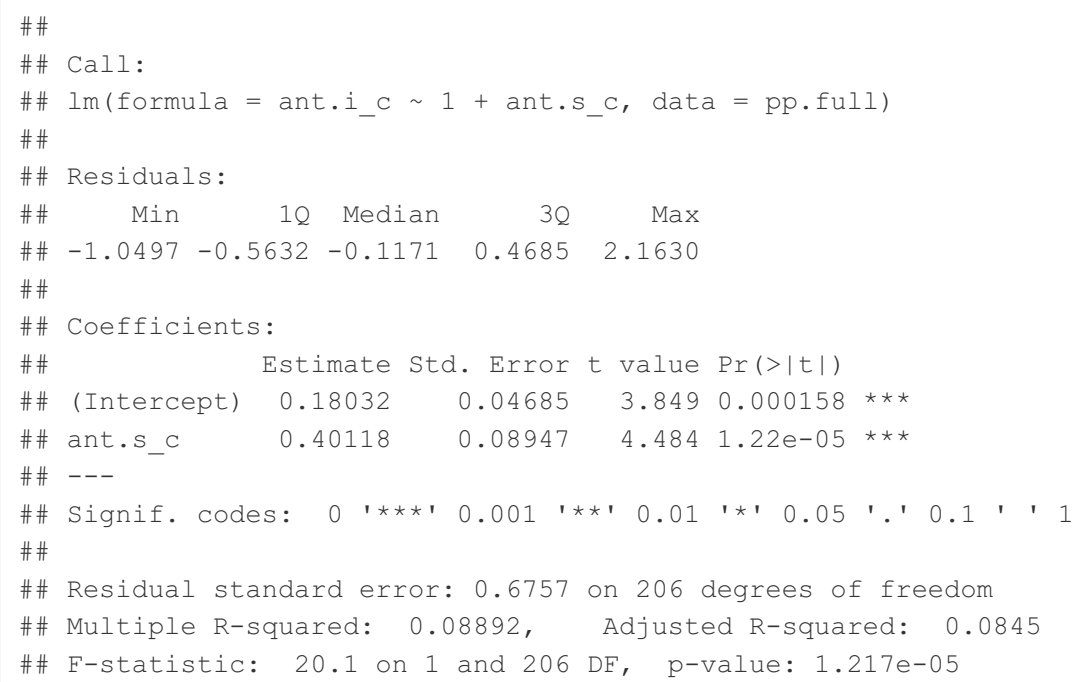

\begin{tabular}{|c|c|}
\hline \#\# & $2.5 \div 97.5 \%$ \\
\hline \#\# (Intercept) & $0.088 \quad 0.273$ \\
\hline \#\# ant.s_c & 0.578 \\
\hline
\end{tabular}

\#\# Moderation model

ant.model $2=\operatorname{lm}($ ant.i_c $\sim$ ant.s_c*gen.z, data $=$ pp.full) summary (ant.model2)

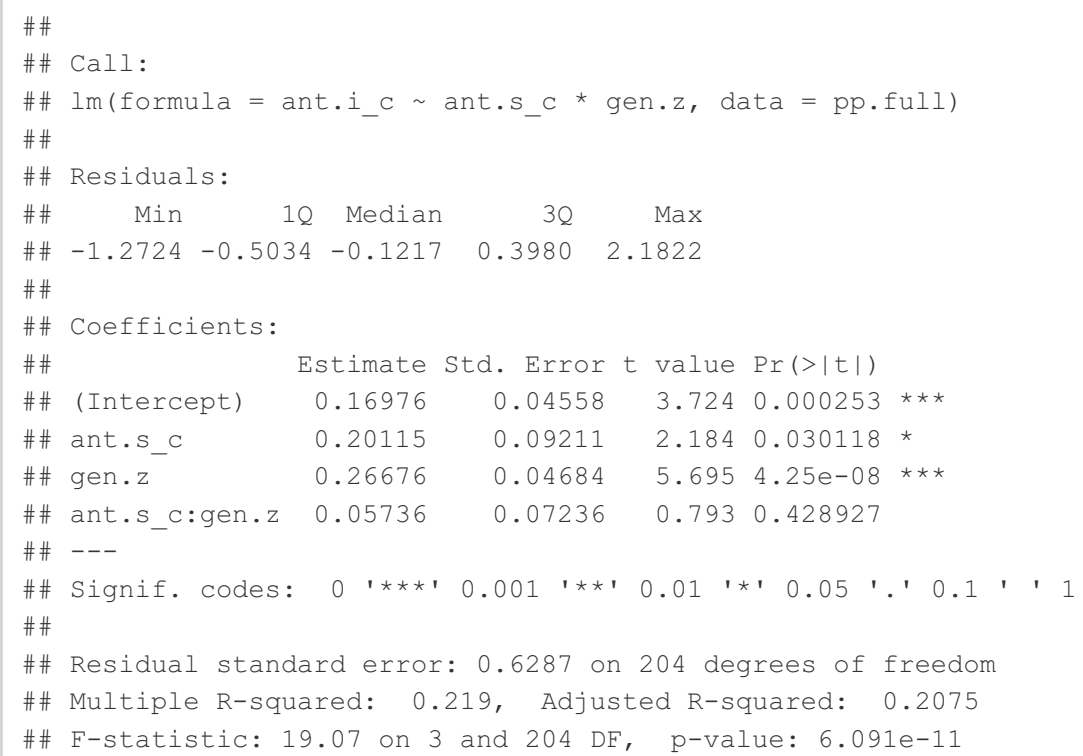




\begin{tabular}{|rrr}
\hline$\# \#$ & $2.5 \%$ & $97.5 \%$ \\
$\# \#$ (Intercept) & 0.164 & 0.305 \\
$\# \#$ dis.s_c & 0.499 & 0.738 \\
\hline
\end{tabular}

\#\# Moderation model

dis.model $2=\operatorname{lm}($ dis.i_c ${ } \sim$ dis.s_c*gen.z, data $=$ pp.full) summary (dis.model2)

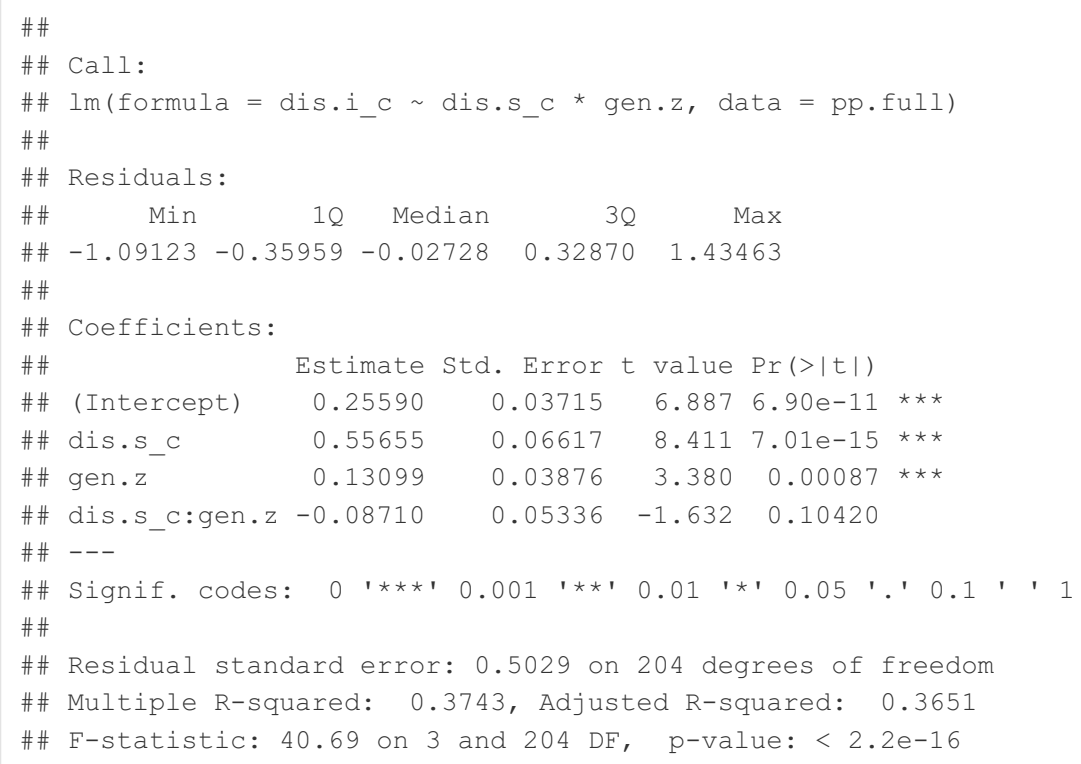

$\begin{array}{llrr}\text { \#\# } & & 2.5 \% & 97.5 \% \\ \text { \#\# } \text { (Intercept) } & 0.183 & 0.329 \\ \text { \#\# dis.s_c } & 0.426 & 0.687 \\ \text { \#\# gen.z } & 0.055 & 0.207 \\ \text { \#\# dis.s_c:gen.z } & -0.192 & 0.018\end{array}$

anova (dis.model1, dis.model2)

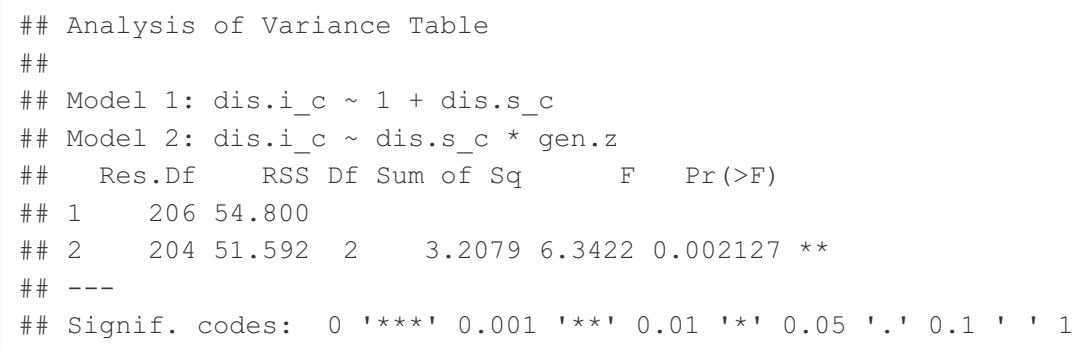




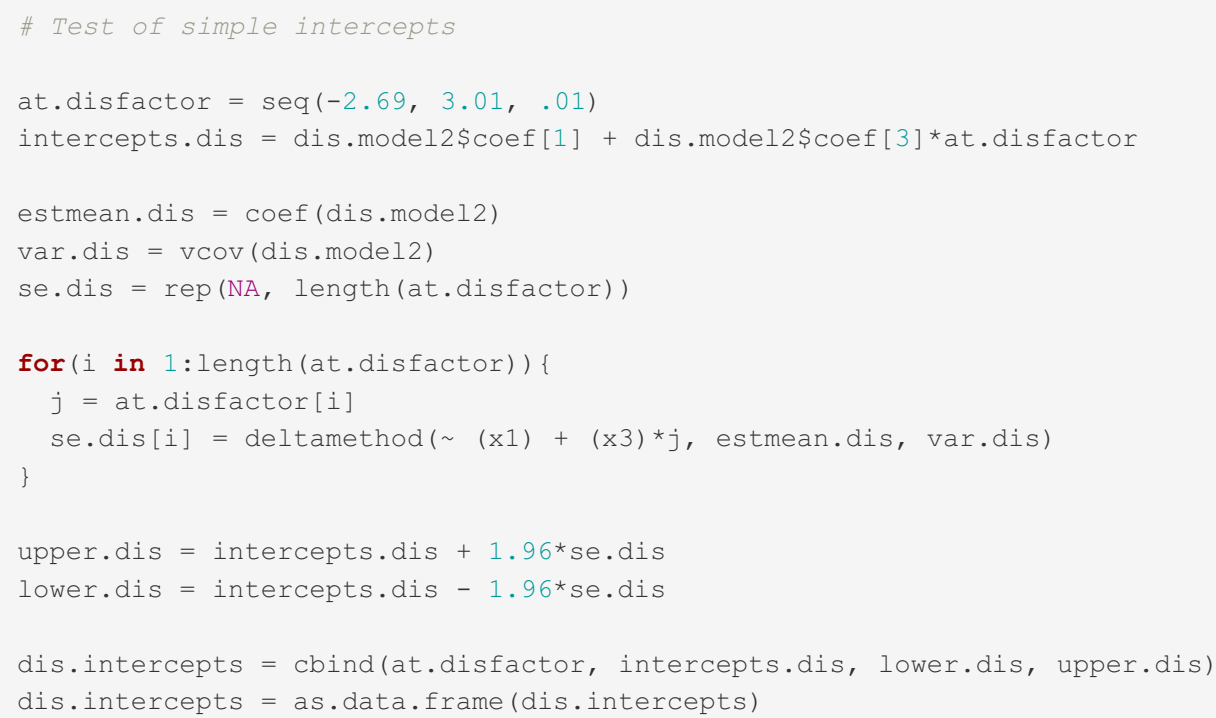

$\begin{array}{rrr}\text { \#\# } & 2.5 \% & 97.5 \% \\ \# \# \text { (Intercept) } & -0.036 & 0.109 \\ \text { \#\# p.s_c } & 0.112 & 0.384\end{array}$


\#\# Call:

\#\# $\operatorname{lm}($ formula $=p . i c \sim p . s, c * g e n . z$, data $=p p . f u l l)$

\#\#

\#\# Residuals:

\# Min 12 Median $30 \quad$ Max

$\begin{array}{llllll}\# \# & -0.7700 & -0.3966 & -0.1645 & 0.2727 & 1.5398\end{array}$

\#\#

\#\# Coefficients:

\#\# Estimate Std. Error $t$ value $\operatorname{Pr}(>|t|)$

$\begin{array}{lllll}\text { \#\# (Intercept) } & 0.05305 & 0.03649 & 1.454 & 0.1476\end{array}$

$\begin{array}{lllll}\text { \#\# p.s_c } & 0.16604 & 0.06970 & 2.382 & 0.0181\end{array}$ *

\#\# gen.z $\quad 0.19101 \quad 0.03654 \quad 5.228 \quad 4.23 e-07 * * *$

$\begin{array}{llllll}\text { \#\# p.s c:gen.z } & -0.10326 & 0.07101 & -1.454 & 0.1475\end{array}$

\#\#---

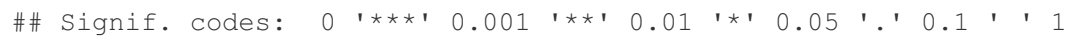

\#\#

\#\# Residual standard error: 0.5005 on 204 degrees of freedom

\#\# Multiple R-squared: 0.1744, Adjusted R-squared: 0.1623

\#\# F-statistic: 14.37 on 3 and 204 DF, p-value: $1.575 e-08$

round (confint (p.model2), 3)

\begin{tabular}{rrr}
\hline$\# \#$ & $2.5 \div$ & $97.5 \%$ \\
$\# \#$ (Intercept) & -0.019 & 0.125 \\
$\# \#$ p.s_c & 0.029 & 0.303 \\
$\# \#$ gen.z & 0.119 & 0.263 \\
$\# \#$ p.s_c:gen.z & -0.243 & 0.037
\end{tabular}

anova (p.model1, p.model2)

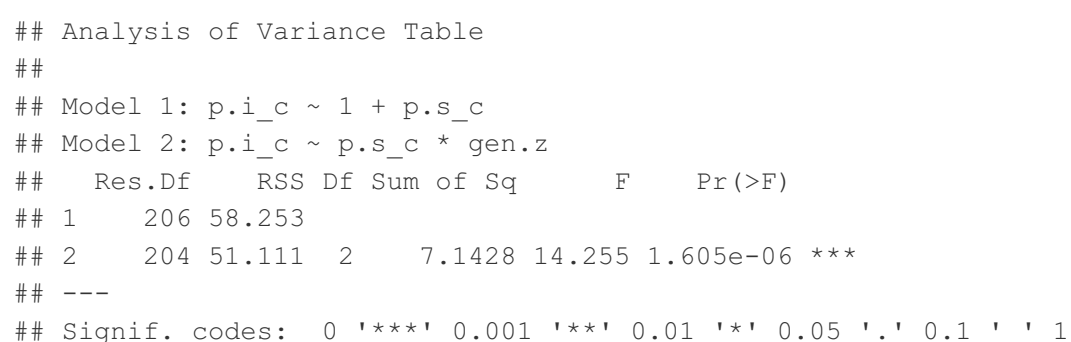

\# Test of simple intercepts

at.pfactor $=\operatorname{seq}(-2.69,3.01, .01)$

intercepts.p $=\mathrm{p} \cdot \operatorname{model} 2 \$ \operatorname{coef}[1]+\mathrm{p} \cdot \operatorname{model} 2 \$ \operatorname{coef}[3] \star a t \cdot p$ factor

estmean.p $=\operatorname{coef}(p \cdot \operatorname{mode} 12)$

$\operatorname{var} \cdot \mathrm{p}=\operatorname{vcov}(\mathrm{p} \cdot \operatorname{model} 2)$

se.p $=\operatorname{rep}(\mathrm{NA}$, length (at.pfactor))

for ( $i$ in 1:length (at.pfactor)) \{

$j=$ at.pfactor $[i]$

se.p[i] = deltamethod $(\sim(x 1)+(x 3) \star j$, estmean.p, var.p $)$

upper.p $=$ intercepts.p $+1.96 *$ se.p

lower.p $=$ intercepts.p $-1.96 *$ se.p

$\mathrm{p}$.intercepts $=\mathrm{cbind}$ (at.pfactor, intercepts.p, lower.p, upper.p)

$\mathrm{p}$.intercepts $=$ as.data.frame $(\mathrm{p}$. intercepts $)$ 
pp.full\$det.s_c = pp.full\$DETACH.s - mean (pp.full\$DETACH.s)

pp.full\$det.i_c $=$ pp.full\$DETACH.i - mean (pp.full\$DETACH.s)

\#\# Base model

$\operatorname{det}$. model $1=\operatorname{lm}\left(\operatorname{det} . \dot{i} \mathrm{c} \sim 1+\operatorname{det} . \mathrm{s}_{\mathbf{c}}\right.$, data $=$ pp.full $)$

summary (det.model1)

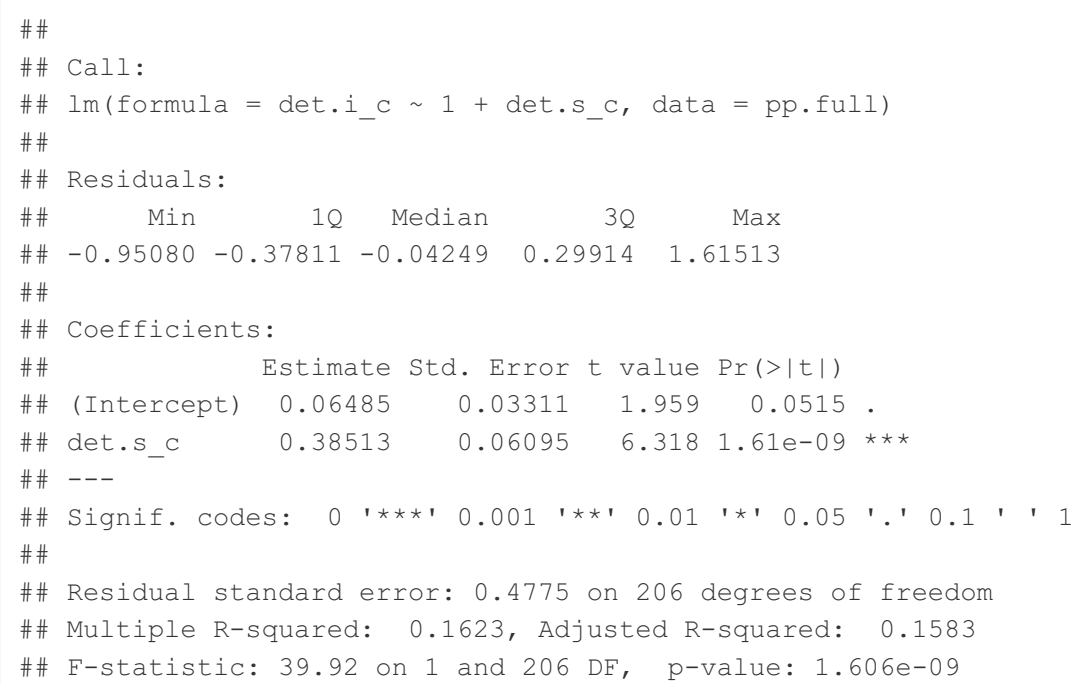

round (confint (det.model1), 3)

\begin{tabular}{lrrr}
$\# \#$ & $2.5 \div$ & $97.5 \%$ \\
$\# \#$ & (Intercept) & 0.000 & 0.130 \\
$\# \#$ det.s_c & 0.265 & 0.505 \\
\hline
\end{tabular}

\#\# Moderation model

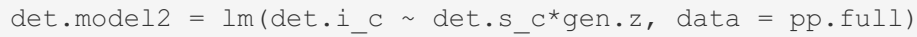
summary (det.model2)

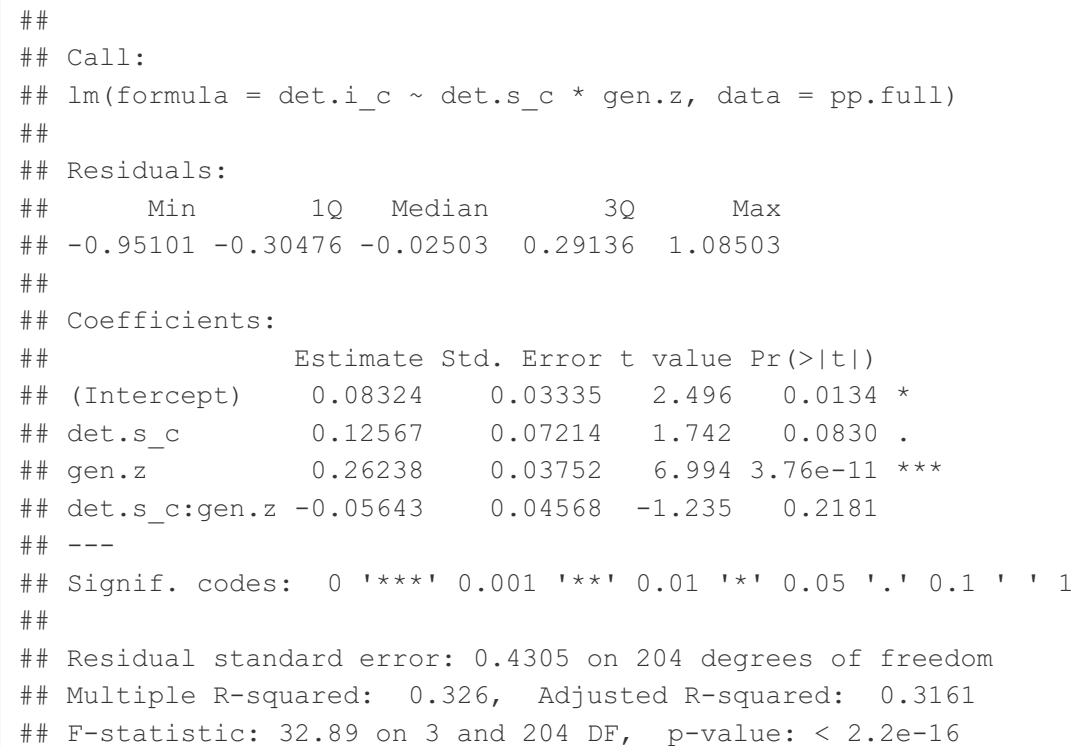




$\begin{array}{llrr}\text { \#\# } & & 2.5 \% & 97.5 \% \\ \text { \#\# (Intercept) } & 0.017 & 0.149 \\ \text { \#\# det.s_c } & -0.017 & 0.268 \\ \text { \#\# gen.z } & 0.188 & 0.336 \\ \text { \#\# det.s_c:gen.z } & -0.146 & 0.034\end{array}$

anova (det.model1, det.model2)

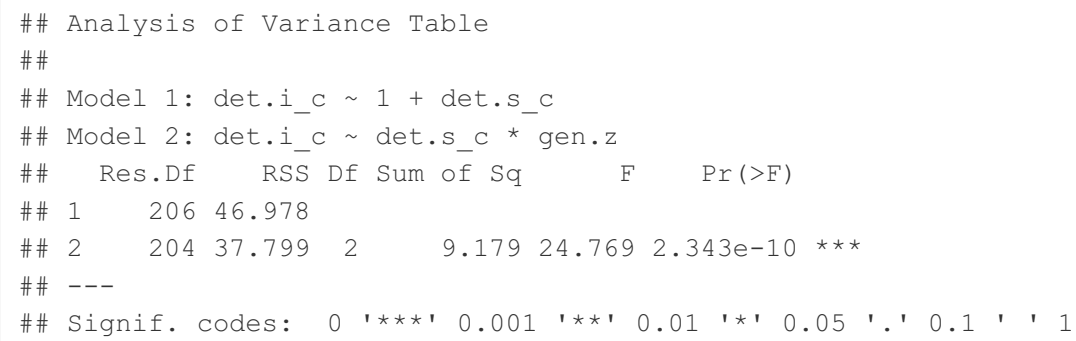

\section{Self-other agreement for PID-5 facets}

\#\# Anhedonia \#\#

t.test (pp.full\$anhedonia.s, pp.full\$anhedonia.i, paired = T)

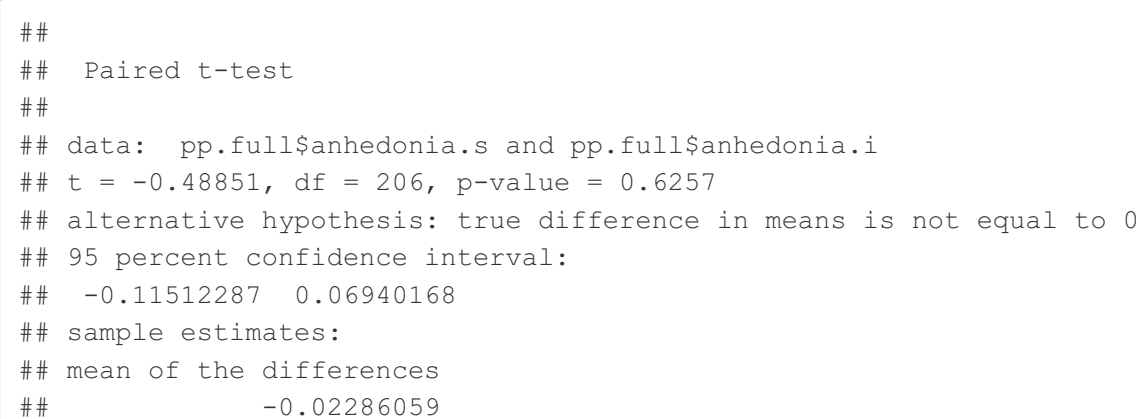


\#\# [1] 0.03395379

$\operatorname{pnorm}(.034)$

\#\# [1] 0.5135614

$(2 * \operatorname{pnorm}((-\operatorname{abs}(.034)) / 2))$

\#\# [1] 0.9864366

print(corr.test(pp.full\$anhedonia.s, pp.full\$anhedonia.i, method = "spearman"), short = F, digits = 3)

\#\# Call:corr.test $(x=$ pp.full\$anhedonia.s, $y=$ pp.full\$anhedonia.i, method = "spearman")

\#\# Correlation matrix

\#\# [1] 0.433

\#\# Sample Size

\#\# [1] 207

\#\# Probability values adjusted for multiple tests.

\#\# [1] 0

\#\#

\#\# Confidence intervals based upon normal theory. To get bootstrapped values, try cor.ci

\#\# raw.lower raw.r raw.upper raw.p lower.adj upper.adj

$\begin{array}{lllllll}\text { \#\# NA-NA } & 0.315 & 0.433 & 0.537 & 0 & 0.315 & 0.537\end{array}$

\# Base model

pp.full\$anhedonia.s_c $=$ pp.full\$anhedonia.s - mean(pp.full\$anhedonia.s)

pp.full\$anhedonia.i_c $=$ pp.full\$anhedonia.i - mean(pp.full\$anhedonia.s)

$\operatorname{anh} 1=\operatorname{lm}($ anhedonia.i_c $\sim 1+$ anhedonia.s_c, data $=$ pp.full)

summary (anh1)

\#\#

\#\# Call:

\#\# $\operatorname{lm}($ formula $=$ anhedonia.i_c $\sim 1+$ anhedonia.s_c, data = pp.full)

\#\#

\#\# Residuals:

\#\# Min 10 Median $30 \quad$ Max

$\begin{array}{llllll}\text { \#\# } & -1.10036 & -0.43169 & -0.07911 & 0.29706 & 1.99401\end{array}$

\#\#

\#\# Coefficients:

\#\# Estimate Std. Error $t$ value $\operatorname{Pr}(>|t|)$

$\begin{array}{lllll}\text { \#\# (Intercept) } & 0.02507 & 0.03827 & 0.655 & 0.513\end{array}$

\#\# anhedonia.s_c $0.39625 \quad 0.05949 \quad 6.660 \quad 2.46 \mathrm{e}-10 * * *$

\#\#---

\#\# Signif. codes: 0 '***' 0.001 '**' $0.011^{\prime *} 0.05$ '.' 0.1 ' ' 1

\#\#

\#\# Residual standard error: 0.5506 on 205 degrees of freedom

\#\# (1 observation deleted due to missingness)

\#\# Multiple R-squared: 0.1779, Adjusted R-squared: 0.1739

\#\# F-statistic: 44.36 on 1 and 205 DF, p-value: 2.462e-10

round (confint (anh1), 3)

$\begin{array}{lrr}\text { \#\# } & 2.5 \div 97.5 \% \\ \text { \#\# (Intercept) } & -0.050 & 0.101 \\ \text { \#\# anhedonia.s_c } & 0.279 & 0.514\end{array}$

\#\# anhedonia.s C $0.279 \quad 0.514$

\# Moderation model

anh2 $=$ lm(anhedonia.i_c $\sim$ anhedonia.s_c*gen.z, data $=$ pp.full)

summary (anh2) 
\#\# Call:

\#\# $\operatorname{lm}($ formula $=$ anhedonia.i $c \sim$ anhedonia.s $c *$ gen.z, data $=$ pp.full)

\#\#

\#\# Residuals:

\#\# Min 12 Median $30 \quad$ Max

$\begin{array}{llllll}\# \# & -0.9583 & -0.3655 & -0.0824 & 0.3417 & 1.5574\end{array}$

\#\#

\#\# Coefficients:

\#\# Estimate Std. Error $t$ value $\operatorname{Pr}(>|t|)$

$\begin{array}{lllll}\text { \#\# (Intercept) } & 0.05376 & 0.03964 & 1.356 & 0.1765\end{array}$

$\begin{array}{lllll}\text { \#\# anhedonia.s_c } & 0.17252 & 0.06942 & 2.485 & 0.0138 \text { * }\end{array}$

$\begin{array}{lllll}\text { \# } \text { gen.z } & 0.28293 & 0.04326 & 6.540 & 4.9 e-10\end{array}$ ***

$\begin{array}{lllll}\text { \#\# anhedonia.s_c:gen.z } & -0.07640 & 0.05020 & -1.522 & 0.1295\end{array}$

$\# \#---$

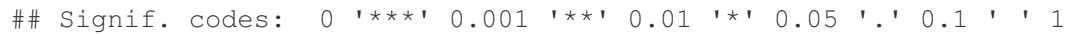

\#\#

\#\# Residual standard error: 0.502 on 203 degrees of freedom

\#\# (1 observation deleted due to missingness)

\#\# Multiple R-squared: 0.3234, Adjusted R-squared: 0.3134

\#\# F-statistic: 32.34 on 3 and 203 DF, p-value: < 2.2e-16

round (confint (anh2), 3)

$\begin{array}{lrr}\text { \#\# } & 2.5 \% & 97.5 \% \\ \text { \#\# (Intercept) } & -0.024 & 0.132 \\ \text { \#\# anhedonia.s_c } & 0.036 & 0.309 \\ \text { \#\# gen.z } & 0.198 & 0.368 \\ \text { \#\# anhedonia.s_c:gen.z } & -0.175 & 0.023\end{array}$

anova (anh1, anh2)

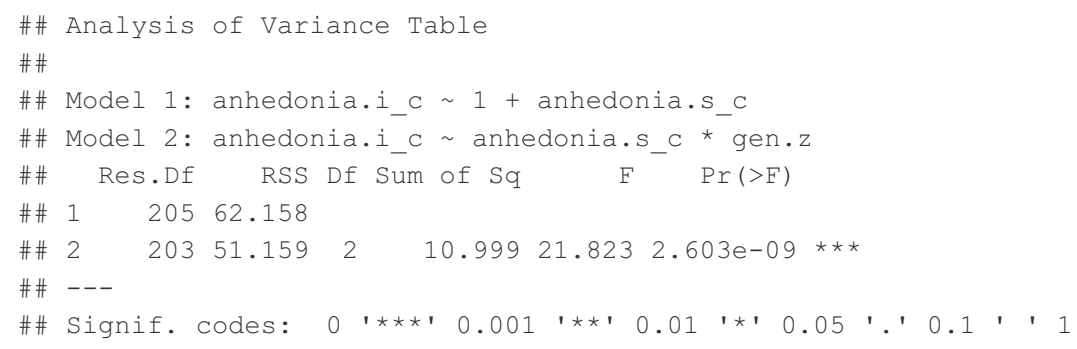

wilcox.test (pp.full\$anxiousness.s, pp.full\$anxiousness.i.ACCURATE, paired = T) 
\#\# [1] 0.07383753

pnorm (.074)

\#\# [1] 0.5294948

$(2 * \operatorname{pnorm}((-\operatorname{abs}(.074)) / 2))$

\#\# [1] 0.970485

print(corr.test(pp.full\$anxiousness.s, pp.full\$anxiousness.i.ACCURATE, method = "spearman"), short = F, digi ts $=3)$

\#\# Call:corr.test $(x=p p . f u l l \$ a n x i o u s n e s s . s, y=p p . f u l l \$ a n x i o u s n e s s . i . A C C U R A T E$,

\#\# method = "spearman")

\#\# Correlation matrix

\#\# [1] 0.452

\#\# Sample Size

\#\# [1] 208

\#\# Probability values adjusted for multiple tests.

\#\# [1] 0

\#\#

\#\# Confidence intervals based upon normal theory. To get bootstrapped values, try cor.ci

\#\# raw.lower raw.r raw.upper raw.p lower.adj upper.adj
\#\# NA-NA
0.3370 .452
$0.554 \quad 0$
0.337
0.554

\# Base model

pp.full\$anxiousness.s c = pp.full\$anxiousness.s - mean(pp.full\$anxiousness.s)

pp.full\$anxiousness.i.ACCURATE_c $=$ pp.full\$anxiousness.i.ACCURATE - mean (pp.full\$anxiousness.s)

anx1 = lm(anxiousness.i.ACCURATE c $1+$ anxiousness.s $c$, data $=$ pp.full)

summary $(\operatorname{anx} 1)$

\#\#

\# Call:

\#\# lm(formula = anxiousness.i.ACCURATE c $\sim 1+$ anxiousness.s c,

\#\# data $=$ pp.full

\#\#

\#\# Residuals:

\#\# Min 10 Median $30 \quad$ Max

$\begin{array}{llllll}\# \# & -1.4485 & -0.5151 & -0.1814 & 0.4740 & 2.0074\end{array}$

\#\#

\#\# Coefficients:

\#\# Estimate Std. Error $t$ value $\operatorname{Pr}(>|t|)$

$\begin{array}{lllll}\text { \#\# (Intercept) } & -0.05783 & 0.04681 & -1.235 & 0.218\end{array}$

\#\# anxiousness.s_c $0.46129 \quad 0.06326 \quad 7.292 \quad 6.43 e-12$ ***

\#\# ---

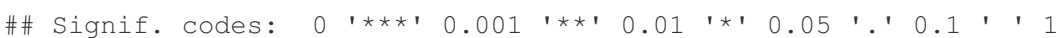

\#\#

\#\# Residual standard error: 0.6752 on 206 degrees of freedom

\#\# Multiple R-squared: 0.2052, Adjusted R-squared: 0.2013

\#\# F-statistic: 53.18 on 1 and 206 DF, p-value: 6.426e-12

round (confint $(\operatorname{anx} 1), 3)$

$\begin{array}{llrr}\text { \#\# } & 2.5 \% & 97.5 \% \\ \text { \#\# (Intercept) } & -0.150 & 0.034 \\ \text { \#\# anxiousness.s_c } & 0.337 & 0.586\end{array}$


\# Moderation model

anx2 = lm(anxiousness.i.ACCURATE_c anxiousness.s_C*gen.z, data $=$ pp.full)

summary (anx2)

\section{\#\#}

\#\# Call:

\#\# Im(formula = anxiousness.i.ACCURATE_c $\sim$ anxiousness.s_c * gen.z,

\#\# data $=$ pp.full)

\#\#

\#\# Residuals:

\#\# Min $\quad 1 Q \quad$ Median $3 Q \quad$ Max

$\begin{array}{llllll}\# \# & -1.41765 & -0.42661 & -0.06202 & 0.36268 & 1.80343\end{array}$

\#\#

\#\# Coefficients:

\#\# Estimate Std. Error $t$ value $\operatorname{Pr}(>|t|)$

$\begin{array}{lllll}\text { \#\# (Intercept) } & -0.07647 & 0.04762 & -1.606 & 0.110\end{array}$

\#\# anxiousness.s_c $\quad 0.18181 \quad 0.07088 \quad 2.565 \quad 0.011$ *

\#\# gen.z $0.35704 \quad 0.05020 \quad 7.1121 .89 e-11 * * *$

$\begin{array}{llllll}\text { \# } \text { anxiousness.s_c:gen.z } & 0.04648 & 0.05575 & 0.834 & 0.405\end{array}$

\#\#---

\#\# Signif. codes: 0 '***' 0.001 '**' 0.01 '*' 0.05 '.' 0.1 ' ' 1

\#\#

\#\# Residual standard error: 0.6063 on 204 degrees of freedom

\#\# Multiple R-squared: 0.3652, Adjusted R-squared: 0.3559

\#\# F-statistic: 39.13 on 3 and 204 DF, p-value: < 2.2e-16

round (confint (anx2), 3)

$\begin{array}{lrr}\text { \#\# } & 2.5 \% & 97.5 \% \\ \text { \#\# (Intercept) } & -0.170 & 0.017 \\ \text { \#\# anxiousness.s_c } & 0.042 & 0.322 \\ \text { \#\# gen.z } & 0.258 & 0.456 \\ \text { \#\# anxiousness.s_c:gen.z } & -0.063 & 0.156\end{array}$

anova (anx1, anx2)

\#\# Analysis of Variance Table

\#\#

\#\# Model 1: anxiousness.i.ACCURATE_c $\sim 1+$ anxiousness.s_c

\#\# Model 2: anxiousness.i.ACCURATE c $\sim$ anxiousness.s $c$ * gen.z

\#\# Res.Df RSS Df Sum of Sq F $\operatorname{Pr}(>\mathrm{F})$

\#\# $1206 \quad 93.901$

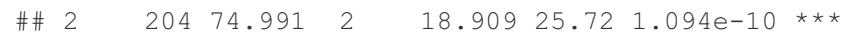

\#\#---

\#\# Signif. codes: 0 '***' 0.001 '**' 0.01 '*' 0.05 '.' 0.1 ' ' 1

\#\# Attention seeking \#\#

t.test (pp.full\$attentionseeking.s, pp.full\$attentionseeking.i, paired $=\mathrm{T}$ )

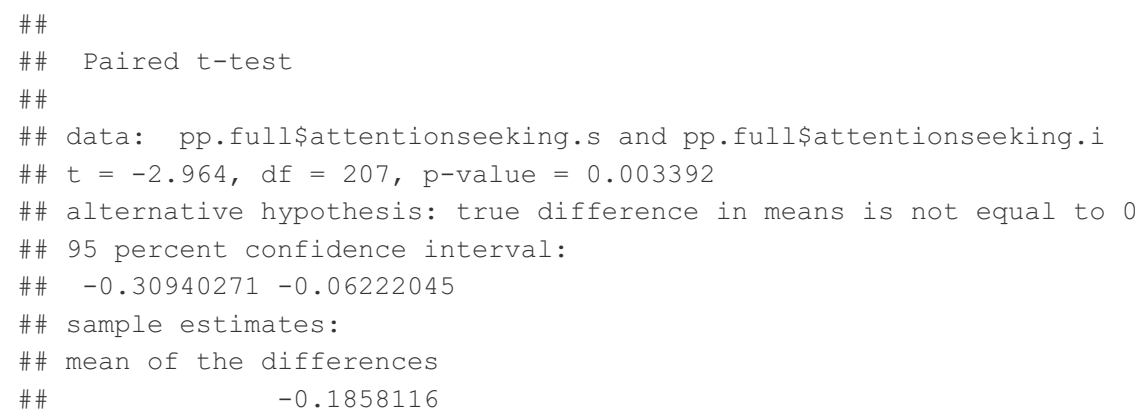

wilcox.test (pp.full\$attentionseeking.s, pp.full\$attentionseeking.i, paired = T) 
\#\# Wilcoxon signed rank test with continuity correction

\#\# data: pp.full\$attentionseeking.s and pp.full\$attentionseeking.i

\#\# $\mathrm{V}=7332.5, \mathrm{p}$-value $=0.009006$

\#\# alternative hypothesis: true location shift is not equal to 0

\section{$(2.964 /$ sqrt (208))}

\#\# [1] 0.2055164

$\operatorname{pnorm}(.206)$

\#\# [1] 0.5816045

$(2 * \operatorname{pnorm}((-\operatorname{abs}(.206)) / 2))$

\# \# [1] 0.917963

print(corr.test (pp.full\$attentionseeking.s, pp.full\$attentionseeking.i, method = "spearman") , short $=F$, digits $=3$ )

\#\# Call:corr.test $(x=p p . f u l l \$ a t t e n t i o n s e e k i n g . s, y=p p . f u l l \$ a t t e n t i o n s e e k i n g \cdot i$,

\#\# method = "spearman")

\#\# Correlation matrix

\#\# [1] 0.236

\#\# Sample Size

\#\# [1] 208

\#\# Probability values adjusted for multiple tests.

\#\# [1] 0.001

\#\#

\# Confidence intervals based upon normal theory. To get bootstrapped values, try cor.ci

\#\# raw.lower raw.r raw.upper raw.p lower.adj upper.adj
\#\# NA-NA
0.1030 .236
0.360 .001
0.103
0.36

\# Base model

pp.full\$attentionseeking.s_c = pp.full\$attentionseeking.s - mean(pp.full\$attentionseeking.s) pp.full\$attentionseeking.i_c = pp.full\$attentionseeking.i - mean(pp.full\$attentionseeking.s)

att $1=\operatorname{lm}($ attentionseeking.i_c $\sim 1+$ attentionseeking.s_c, data $=$ pp.full)

summary (att1)

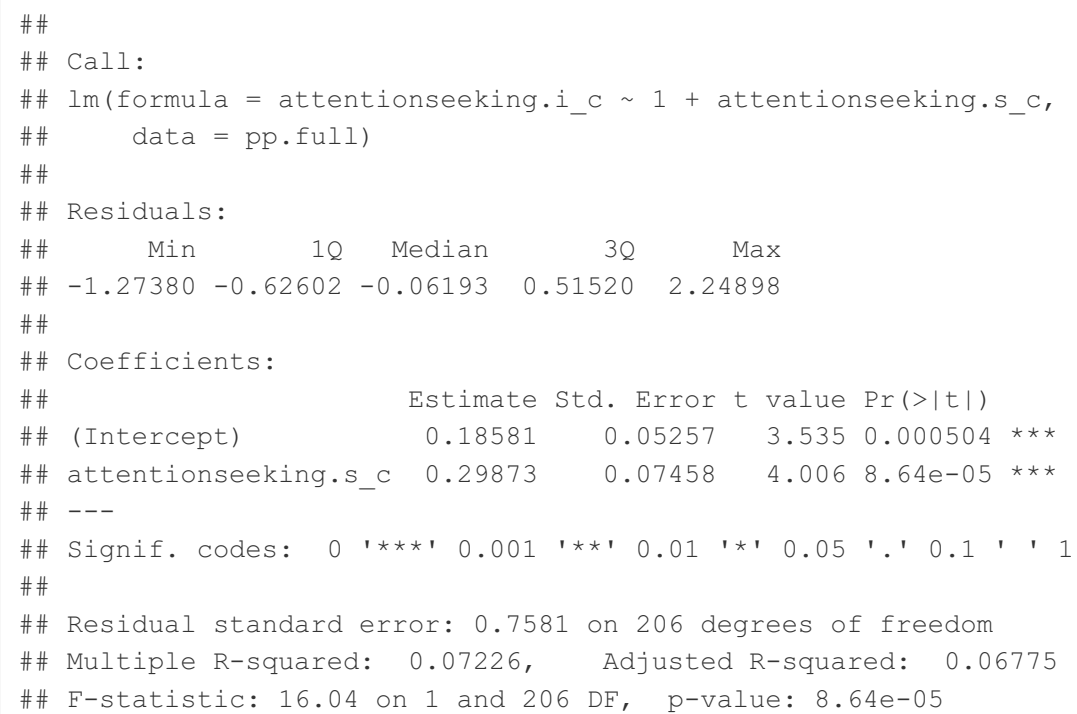




$\begin{array}{lrr}\# \# & 2.5 \% & 97.5 \% \\ \# \# \text { (Intercept) } & 0.082 & 0.289 \\ \text { \#\# attentionseeking.s_c } & 0.152 & 0.446\end{array}$

\# Moderation model

att2 $=\operatorname{lm}\left(\right.$ attentionseeking.i_c $\sim$ attentionseeking.s_c $c^{\star} g e n . z$, data $=$ pp.full)

summary (att2)

\section{\#\#}

\#\# Call:

\#\# $\operatorname{lm}($ formula $=$ attentionseeking.i_c $\sim$ attentionseeking.s_c * gen.z,

\#\# data $=$ pp.full)

\#\#

\#\# Residuals:

\#\# Min $\quad 10$ Median $30 \quad$ Max

$\begin{array}{llllll}\# \# & -1.35375 & -0.60015 & -0.05232 & 0.54470 & 2.16928\end{array}$

\#\#

\#\# Coefficients:

\#\# Estimate Std. Error $t$ value Pr $(>|t|)$

$\begin{array}{lllll}\text { \# } \text { (Intercept) } & 0.18307 & 0.05202 & 3.519 & 0.000534\end{array}$ ***

$\begin{array}{lllll}\text { \# \# attentionseeking.s_c } & 0.26253 & 0.07447 & 3.525 & 0.000522 * * *\end{array}$

$\begin{array}{lllll}\text { \# gen.z } & 0.16109 & 0.05237 & 3.076 & 0.002386\end{array}$ **

$\begin{array}{llllll}\text { \#\# attentionseeking.s_c:gen.z } & 0.02760 & 0.06726 & 0.410 & 0.681966\end{array}$

\#\# ---

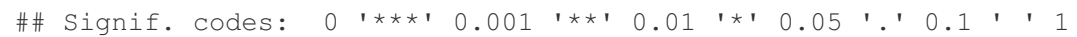

\#\#

\#\# Residual standard error: 0.744 on 204 degrees of freedom

\#\# Multiple R-squared: 0.115, Adjusted R-squared: 0.102

\#\# F-statistic: 8.838 on 3 and $204 \mathrm{DF}$, p-value: $1.553 \mathrm{e}-05$

round (confint (att2), 3)

\begin{tabular}{|lrr|}
\hline$\# \#$ & $2.5 \div$ & $97.5 \%$ \\
$\# \#$ (Intercept) & 0.080 & 0.286 \\
$\# \#$ attentionseeking.s_c & 0.116 & 0.409 \\
$\# \#$ gen.z & 0.058 & 0.264 \\
$\# \#$ attentionseeking.s_c:gen.z & -0.105 & 0.160 \\
\hline
\end{tabular}

anova (att1, att2)

\#\# Analysis of Variance Table

\#\#

\#\# Model 1: attentionseeking.i_c $\sim 1+$ attentionseeking.s c

\#\# Model 2: attentionseeking.i_c $\sim$ attentionseeking.s_c * $g e n . z$

\#\# Res.Df RSS Df Sum of Sq F $\operatorname{Pr}(>\mathrm{F})$

$\begin{array}{llll}\# \# & 1 & 206 & 118.39\end{array}$

\#\# $2 \quad 204 \quad 112.94 \quad 2 \quad 5.4574 \quad 4.9290 .008119$ **

\#\# ---

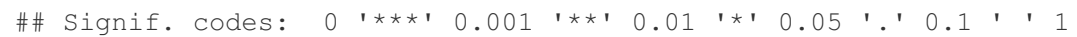

\#\# Callousness \#\#

t.test (pp.full\$callousness.s, pp.full\$callousness.i, paired $=\mathrm{T}$ )

\#\#

\#\# Paired t-test

\#\#

\#\# data: pp.full\$callousness.s and pp.full\$callousness.i

\#\# $t=-5.5883, \mathrm{df}=207, \mathrm{p}$-value $=7.174 \mathrm{e}-08$

\#\# alternative hypothesis: true difference in means is not equal to 0

\#\# 95 percent confidence interval:

\#\# -0.3012704 -0.1441360

\#\# sample estimates:

\#\# mean of the differences

\#\#

$-0.2227032$ 
\#\#

\#\# Wilcoxon signed rank test with continuity correction

\#\#

\#\# data: pp.full\$callousness.s and pp.full\$callousness.i

\#\# $\mathrm{V}=5044, \mathrm{p}$-value $=1.652 \mathrm{e}-06$

\#\# alternative hypothesis: true location shift is not equal to 0

(5.5883/sqrt (208))

\#\# [1] 0.3874789

pnorm (.387)

\#\# [1] 0.6506219

$(2 * \operatorname{pnorm}((-\operatorname{abs}(.387)) / 2))$

\#\# [1] 0.8465674

print(corr.test(pp.full\$callousness.s, pp.full\$callousness.i, method = "spearman"), short = F, digits = 3)

\#\# Call:corr.test $(x=p p . f u l l \$ c a l l o u s n e s s . s, y=p p . f u l l \$ c a l l o u s n e s s . i$,

\#\# method = "spearman")

\#\# Correlation matrix

\#\# [1] 0.356

\#\# Sample Size

\#\# [1] 208

\#\# Probability values adjusted for multiple tests.

\#\# [1] 0

\#\#

\#\# Confidence intervals based upon normal theory. To get bootstrapped values, try cor.ci

\#\# raw.lower raw.r raw.upper raw.p lower.adj upper.adj

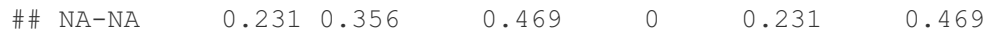

\# Base model

pp.full\$callousness.s_c = pp.full\$callousness.s - mean(pp.full\$callousness.s)

pp.full\$callousness.i_c $=$ pp.full\$callousness.i - mean(pp.full\$callousness.s)

cal1 = lm(callousness.i_c 1 + callousness.s_c, data = pp.full)

summary (cal1)

\#\#

\#\# Call:

\#\# lm(formula = callousness.i_c $\sim 1+$ callousness.s_c, data $=$ pp.full)

\#\#

\#\# Residuals:

\#\# Min 10 Median $30 \quad$ Max

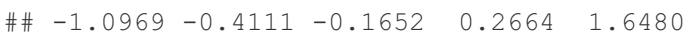

\#\#

\#\# Coefficients:

\#\# Estimate Std. Error $t$ value $\operatorname{Pr}(>|t|)$

\#\# (Intercept) $\quad 0.22270 \quad 0.03740 \quad 5.9551 .11$ e-08 ***

\#\# callousness.s_c $0.51431 \quad 0.09005 \quad 5.7113 .88 e-08 * * *$

\#\#---

\#\# Signif. codes: 0 '***' 0.001 '**' 0.01 '*' 0.05 '.' 0.1 ' ' 1

\#\#

\#\# Residual standard error: 0.5393 on 206 degrees of freedom

\#\# Multiple R-squared: 0.1367, Adjusted R-squared: 0.1325

\#\# F-statistic: 32.62 on 1 and 206 DF, p-value: $3.883 e-08$ 


$\begin{array}{rrrr}\text { \#\# } & 2.5 \div & 97.5 \% \\ \text { \#\# } & \text { (Intercept) } & 0.149 & 0.296 \\ \text { \#\# } & \text { callousness.s_c } & 0.337 & 0.692\end{array}$

\# Moderation model

cal2 = lm(callousness.i_c $\sim$ callousness.s_c*gen.z, data = pp.full)

summary (cal2)

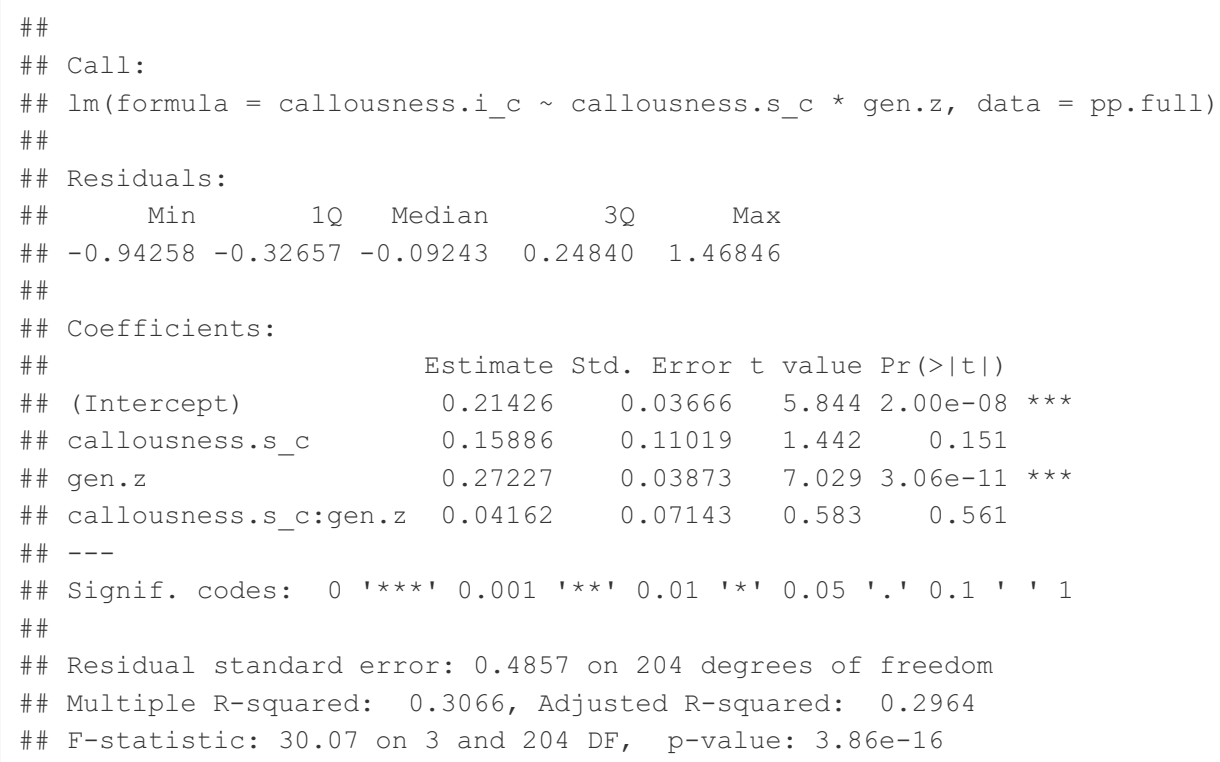

\begin{tabular}{|c|c|c|c|}
\hline \#\# & (Intercept) & callousness.s_c & gen.z \\
\hline \#\# & 0.214 & 0.159 & 0.272 \\
\hline & S.S_c:gen.z & & \\
\hline \#\# & 0.042 & & \\
\hline
\end{tabular}

round (confint (cal2), 3)

\begin{tabular}{|rrr|}
\hline$\# \#$ & $2.5 \div$ & $97.5 \%$ \\
$\# \#$ (Intercept) & 0.142 & 0.287 \\
$\#$ \# callousness.s_c & -0.058 & 0.376 \\
$\#$ gen.z & 0.196 & 0.349 \\
$\#$ \# callousness.s_c:gen.z & -0.099 & 0.182 \\
\hline
\end{tabular}

anova (cal1, cal2)

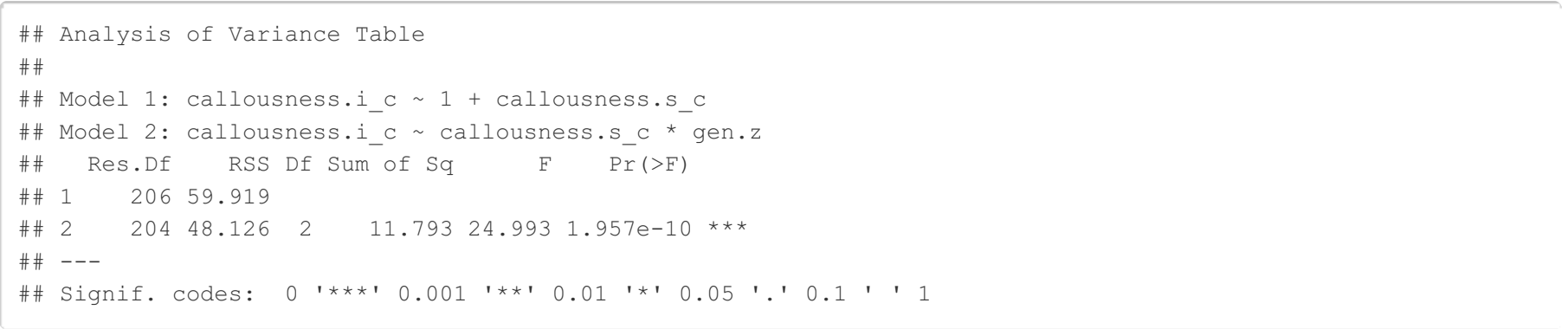

\section{\#\# Deceitfulness \#\#}

t.test (pp.full\$deceitfulness.s, pp.full\$deceitfulness.i, paired $=T$ ) 


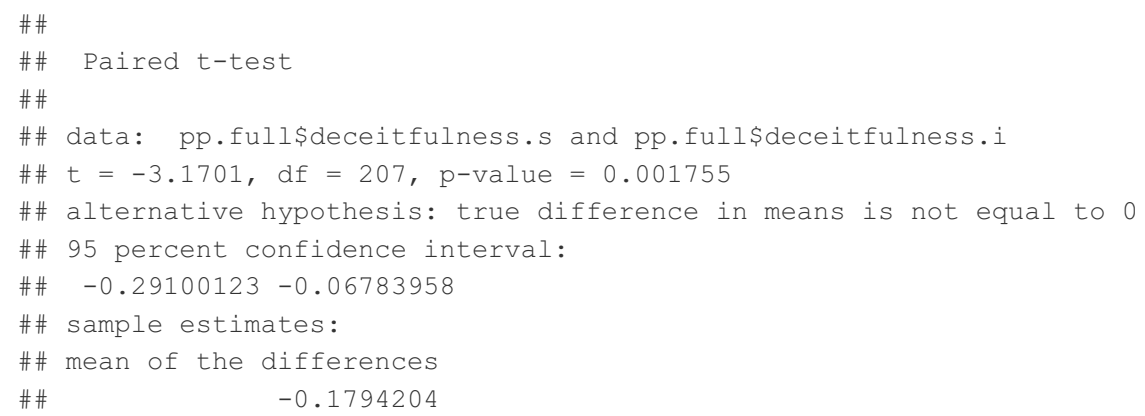

\#\# [1] 0.2198069

$\operatorname{pnorm}(.220)$

\#\# [1] 0.5870644

$(2 * \operatorname{pnorm}((-\operatorname{abs}(.220)) / 2))$

\#\# [1] 0.9124094

print(corr.test(pp.full\$deceitfulness.s, pp.full\$deceitfulness.i, method = "spearman"), short = F, digits = 3)

\#\# Call:corr.test $(x=p p . f u l l \$ d e c e i t f u l n e s s . s, y=p p . f u l l \$ d e c e i t f u l n e s s . i$,

\#\# method = "spearman")

\#\# Correlation matrix

\#\# [1] 0.214

\#\# Sample Size

\#\# [1] 208

\#\# Probability values adjusted for multiple tests.

\#\# [1] 0.002

\#\#

\#\# Confidence intervals based upon normal theory. To get bootstrapped values, try cor.ci

\#\# raw.lower raw.r raw.upper raw.p lower.adj upper.adj
\#\# NA-NA
0.080 .214
0.340 .002
0.08
0.34

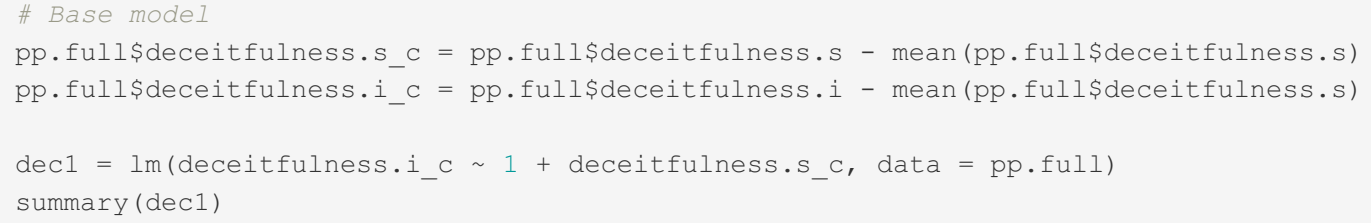


\# Call:

\#\# $\operatorname{lm}($ formula $=$ deceitfulness.i $c \sim 1$ +deceitfulness.s c, data = pp.full)

\#\#

\#\# Residuals:

\#\# Min 10 Median $30 \quad$ Max

$\begin{array}{llllll}\# \# & -1.2451 & -0.6045 & -0.2002 & 0.5545 & 2.2244\end{array}$

\#\#

\#\# Coefficients:

\#\# Estimate std. Error $t$ value $\operatorname{Pr}(>|t|)$

\#\# (Intercept) $0.17942 \quad 0.05063 \quad 3.5440 .000488 * * *$

\#\# deceitfulness.s_c $0.35589 \quad 0.08875 \quad 4.0108 .49 e-05 * * *$

\#\#---

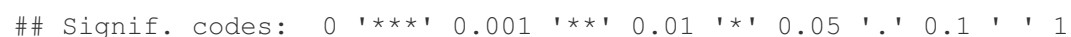

\#\#

\#\# Residual standard error: 0.7302 on 206 degrees of freedom

\#\# Multiple R-squared: 0.07241, Adjusted R-squared: 0.0679

\#\# F-statistic: 16.08 on 1 and 206 DF, p-value: 8.491e-05

round (confint (dec1), 3)

$\begin{array}{lrrr}\text { \#\# } & 2.5 \% & 97.5 \% \\ \text { \#\# (Intercept) } & 0.080 & 0.279 \\ \text { \#\# deceitfulness.s_c } & 0.181 & 0.531\end{array}$

\# Moderation model

dec2 = lm(deceitfulness.i_c $\sim$ deceitfulness.s_c*gen.z, data $=$ pp.full)

summary $(\operatorname{dec} 2)$

\#\#

\#\# Call:

\#\# Im(formula = deceitfulness.i_c $\sim$ deceitfulness.s_c ${ }^{*}$ gen.z, data $=$ pp.full)

\#\#

\#\# Residuals:

\#\# Min 12 Median $30 \quad$ Max

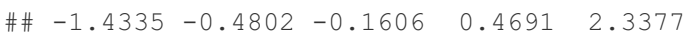

\#\#

\#\# Coefficients:

\#\# Estimate Std. Error $t$ value Pr $(>|t|)$

\#\# (Intercept) $0.16922 \quad 0.04953 \quad 3.4160 .000766$ ***

$\begin{array}{llllll}\text { \# } \text { deceitfulness.s_c } & 0.10164 & 0.09620 & 1.057 & 0.291957\end{array}$

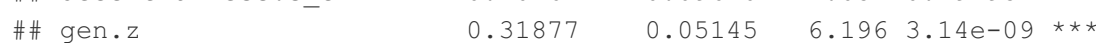

\#\# deceitfulness.s_c:gen.z $0.04309 \quad 0.07110 \quad 0.606 \quad 0.545163$

\#\# ---

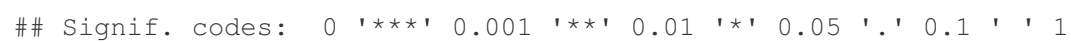

\#\#

\#\# Residual standard error: 0.6718 on 204 degrees of freedom

\#\# Multiple R-squared: 0.2224, Adjusted R-squared: 0.211

\#\# F-statistic: 19.45 on 3 and 204 DF, p-value: $3.932 e-11$

round (confint (dec2), 3)

$\begin{array}{rrr}\text { \#\# } & 2.5 \% & 97.5 \% \\ \# \# \text { (Intercept) } & 0.072 & 0.267 \\ \text { \#\# deceitfulness.s_c } & -0.088 & 0.291 \\ \text { \#\# gen.z } & 0.217 & 0.420 \\ \text { \#\# deceitfulness.s_c:gen.z } & -0.097 & 0.183\end{array}$

anova (dec1, dec2) 


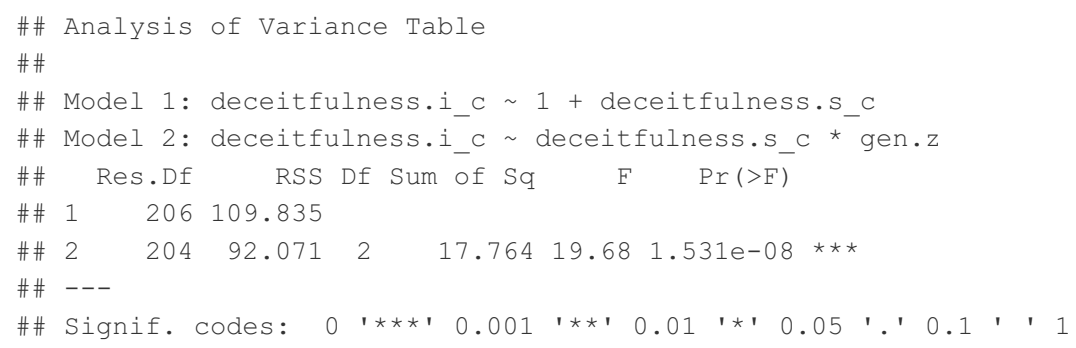

wilcox.test (pp.full\$depressivity.s, pp.full\$depressivity.i, paired = T)

\#\#

\#\# Wilcoxon signed rank test with continuity correction

\#\#

\#\# data: pp.full\$depressivity.s and pp.full\$depressivity.i

\#\# $\mathrm{V}=6513, \mathrm{p}$-value $=0.02635$

\#\# alternative hypothesis: true location shift is not equal to 0

(2.7026/sqrt (207))

\#\# [1] 0.1878437

pnorm (.188)

\#\# [1] 0.5745617

$(2 * \operatorname{pnorm}((-\operatorname{abs}(.188)) / 2))$

\# \# [1] 0.9251092

print(corr.test(pp.full\$depressivity.s, pp.full\$depressivity.i, method = "spearman"), short = F, digits = 3)

\#\# Call:corr.test $(x=p p . f u l l$ depressivity.s, $y=p p . f u l l$ depressivity.i,

\#\# method = "spearman")

\#\# Correlation matrix

\#\# [1] 0.527

\#\# Sample Size

\#\# [1] 207

\#\# Probability values adjusted for multiple tests.

\#\# [1] 0

\#\#

\#\# Confidence intervals based upon normal theory. To get bootstrapped values, try cor.ci

\#\# raw.lower raw.r raw.upper raw.p lower.adj upper.adj

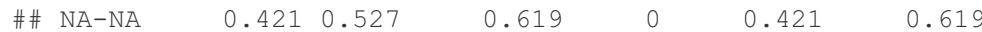


\# Base model

pp.full\$depressivity.s_c = pp.full\$depressivity.s - mean(pp.full\$depressivity.s)

pp.full\$depressivity.i_c $=$ pp.full\$depressivity.i - mean(pp.full\$depressivity.s)

dep1 = lm(depressivity.i_c $\sim 1+$ depressivity.s_c, data = pp.full)

summary (dep1)

\#\#

\#\# Call:

\#\# $\operatorname{lm}$ (formula = depressivity.i_c 1 + depressivity.s_c, data $=$ pp.full)

\#\#

\#\# Residuals:

\#\# Min 10 Median $30 \quad$ Max

$\begin{array}{llllll}\# \# & -1.1901 & -0.3590 & -0.1310 & 0.2852 & 2.2893\end{array}$

\#\#

\#\# Coefficients:

\#\# Estimate Std. Error t value $\operatorname{Pr}(>|t|)$

\#\# (Intercept) $\quad 0.11295 \quad 0.03711 \quad 3.044 \quad 0.00264 * *$

\#\# depressivity.s_c $0.55768 \quad 0.061859 .017<2 e-16 * * *$

\#\#---

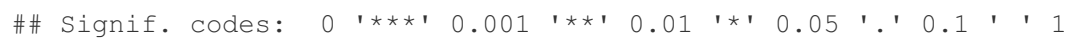

\#\#

\#\# Residual standard error: 0.5339 on 205 degrees of freedom

\#\# (1 observation deleted due to missingness)

\#\# Multiple R-squared: 0.284, Adjusted R-squared: 0.2805

\#\# F-statistic: 81.31 on 1 and 205 DF, p-value: $<2.2 e-16$

round (confint (dep1), 3)

$\begin{array}{lrr}\# \# & 2.5 \% & 97.5 \% \\ \# \# \text { (Intercept) } & 0.040 & 0.186 \\ \# \# \text { depressivity.s_c } & 0.436 & 0.680\end{array}$

\# Moderation model

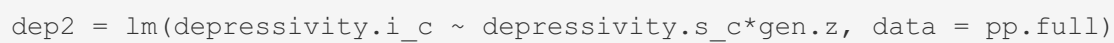

summary (dep2)

\#\#

\#\# Call:

\#\# Im(formula = depressivity.i $\mathrm{c} \sim$ depressivity.s $\mathrm{c} *$ gen.z, data $=$ pp.full)

\#\#

\#\# Residuals:

\#\# Min 10 Median $30 \quad$ Max

$\begin{array}{llllll}\# \# & -1.0156 & -0.3305 & -0.1261 & 0.2839 & 1.7885\end{array}$

\#\#

\#\# Coefficients:

\#\#

Estimate sta. Error t value $\operatorname{Pr}(>|t|)$

$\begin{array}{llllll}\text { \#\# (Intercept) } & 0.112607 & 0.038214 & 2.947 & 0.00359 * *\end{array}$

\#\# depressivity.s_c $\quad 0.358520 \quad 0.074031 \quad 4.8432 .53 e-06 * * *$

$\begin{array}{lllllll}\text { \# gen.z } & 0.235783 & 0.040525 & 5.818 & 2.29 e-08 * * *\end{array}$

$\begin{array}{llllll}\text { \# depressivity.s c:gen.z } & -0.002949 & 0.053713 & -0.055 & 0.95628\end{array}$

\#\# ---

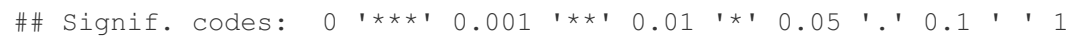

\#\#

\#\# Residual standard error: 0.4967 on 203 degrees of freedom

\# (1 observation deleted due to missingness)

\#\# Multiple R-squared: 0.3864, Adjusted R-squared: 0.3774

\#\# F-statistic: 42.62 on 3 and 203 DF, p-value: < 2.2e-16

round (coef (dep2), 3)

$\begin{array}{lrrr}\# \# & \text { (Intercept) } & \text { depressivity.s_c } & \text { gen.z } \\ \# \# & 0.113 & 0.359 & 0.236\end{array}$

\#\# depressivity.s c:gen.z

\#\#

$-0.003$ 


\begin{tabular}{|lrr|}
\hline$\# \#$ & $2.5 \div$ & $97.5 \%$ \\
$\# \#$ (Intercept) & 0.037 & 0.188 \\
$\# \#$ depressivity.s_c & 0.213 & 0.504 \\
$\# \#$ gen.z & 0.156 & 0.316 \\
\#\# depressivity.s_c:gen.z & -0.109 & 0.103 \\
\hline
\end{tabular}

anova (dep1, dep2)

\#\# Analysis of Variance Table

\#\#

\#\# Model 1: depressivity.i_c $\sim 1+$ depressivity.s_c

\#\# Model 2: depressivity.i_c $\sim$ depressivity.s_c * gen.z

\#\# Res.Df RSS Df Sum of Sq F $\operatorname{Pr}(>\mathrm{F})$

\#\# $1205 \quad 58.432$

$\begin{array}{llllllll}\# 2 & 2 & 203 & 50.073 & 2 & 8.3595 & 16.945 & 1.564 \mathrm{e}-07\end{array}$ ***

\#\#---

\#\# Signif. codes: 0 '***' 0.001 '**' 0.01 '*' 0.05 '.' 0.1 ' ' 1

\#\# Distractibility \#\#

t.test(pp.full\$distractibility.s, pp.full\$distractibility.i, paired $=T$ )

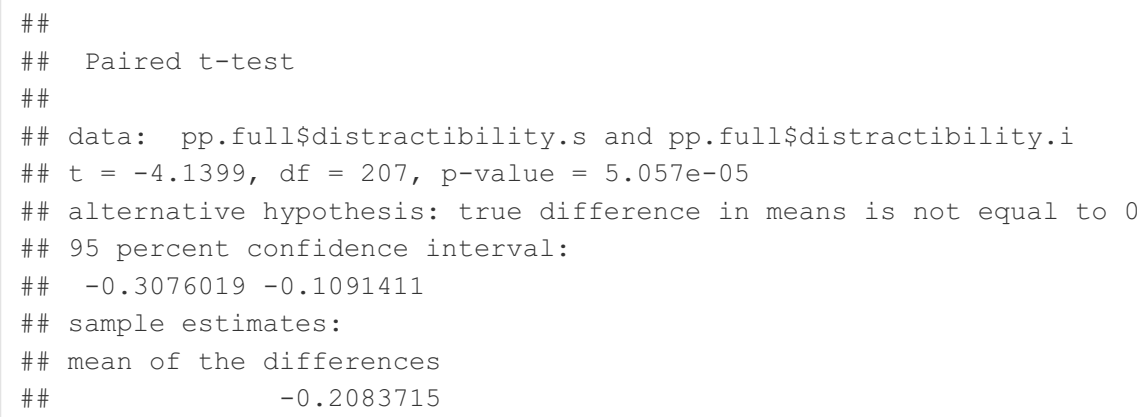

wilcox.test (pp.full\$distractibility.s, pp.full\$distractibility.i, paired $=T$ )

\#\#

\#\# Wilcoxon signed rank test with continuity correction

\#\#

\#\# data: pp.full\$distractibility.s and pp.full\$distractibility.i

\#\# $\mathrm{V}=6438, \mathrm{p}$-value $=0.0001688$

\#\# alternative hypothesis: true location shift is not equal to 0

$(4.1399 / \operatorname{sqrt}(208))$

\#\# [1] 0.2870504

$\operatorname{pnorm}(.287)$

\#\# [1] 0.6129438

$(2 * \operatorname{pnorm}((-\operatorname{abs}(.287)) / 2))$

\#\# [1] 0.8858953

print(corr.test (pp.full\$distractibility.s, pp.full\$distractibility.i, method = "spearman"), short = F, digit $\mathrm{s}=3)$ 


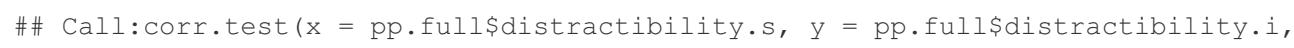

\#\# $\quad$ method = "spearman")

\#\# Correlation matrix

\#\# [1] 0.46

\#\# Sample Size

\#\# [1] 208

\#\# Probability values adjusted for multiple tests.

\#\# [1] 0

\#\#

\#\# Confidence intervals based upon normal theory. To get bootstrapped values, try cor.ci

\#\# raw.lower raw.r raw.upper raw.p lower.adj upper.adj

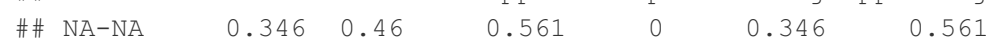

* Base model

pp.full\$distractibility.s_c = pp.full\$distractibility.s - mean(pp.full\$distractibility.s)

pp.full\$distractibility.i_c = pp.full\$distractibility.i - mean(pp.full\$distractibility.s)

dist1 $=\operatorname{lm}($ distractibility.i_c $\sim 1+$ distractibility.s_c, data $=$ pp.full)

summary (dist1)

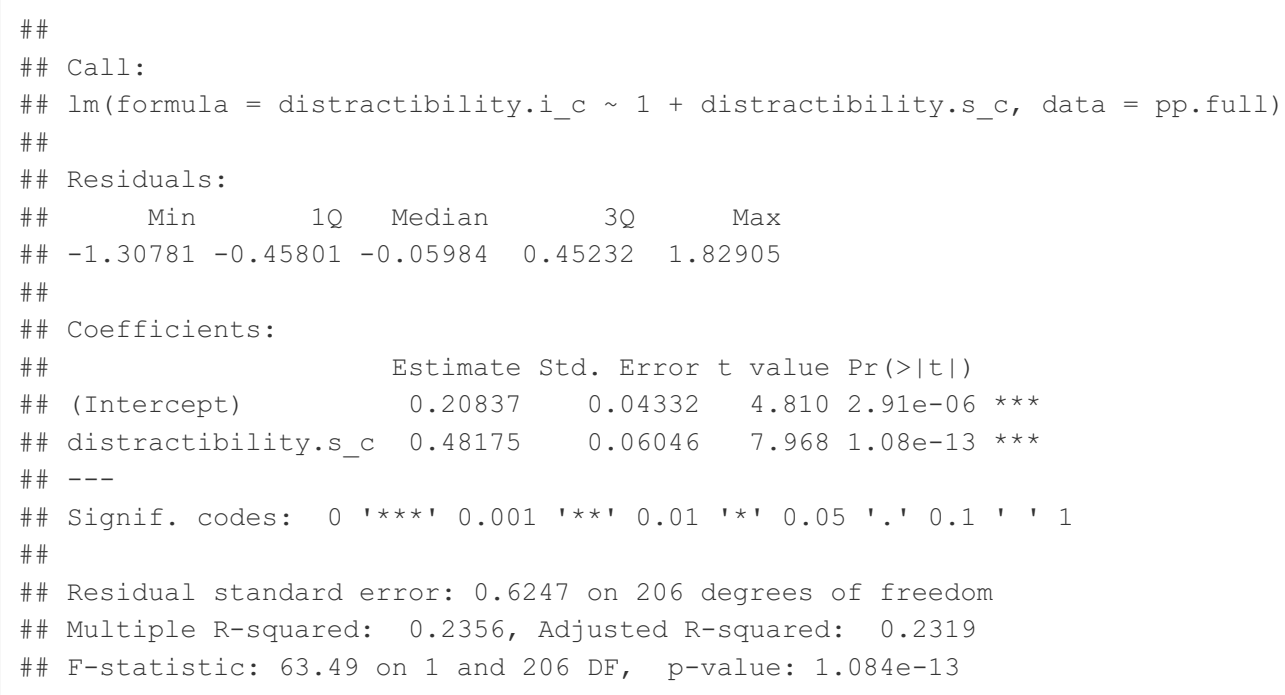

$\begin{array}{lrrr}\text { \#\# } & 2.5 \% & 97.5 \% \\ \# \# \text { (Intercept) } & 0.123 & 0.294 \\ \text { \#\# distractibility.s_c } & 0.363 & 0.601\end{array}$

\# Moderation model

dist2 $=\operatorname{lm}\left(\right.$ distractibility.i_c $\sim$ distractibility.s_c ${ }^{\star} g e n . z$, data $=$ pp.full) summary (dist2) 
\#\# Call:

\#\# $\operatorname{lm}($ formula $=$ distractibility.i $c \sim$ distractibility.s_c * gen.z,

\#\# data $=$ pp.full)

\#\#

\#\# Residuals:

\#\# Min $\quad 12$ Median $30 \quad$ Max

$\begin{array}{llllll}\# \# & -1.25795 & -0.42481 & -0.06599 & 0.41879 & 2.00644\end{array}$

\#\#

\#\# Coefficients:

\#\#

\# \# ( Intercept)

\#\# distractibility.s_c

$\begin{array}{rrrr}\text { Estimate Std. Error } t \text { value } \operatorname{Pr}(>|t|) \\ 0.22846 & 0.04592 & 4.975 & 1.38 \mathrm{e}-06 * \star \star \\ 0.41241 & 0.06568 & 6.279 & 2.01 \mathrm{e}-09 * * * \\ 0.14833 & 0.04777 & 3.105 & 0.00217 * \star \\ -0.06802 & 0.05884 & -1.156 & 0.24903\end{array}$

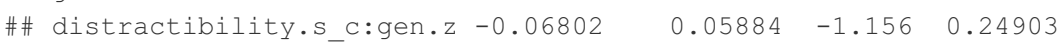

\#\#---

\#\# Signif. codes: 0 '***' 0.001 '**' 0.01 '*' 0.05 '.' 0.1 ' ' 1

\#\#

\#\# Residual standard error: 0.613 on 204 degrees of freedom

\#\# Multiple R-squared: 0.2711, Adjusted R-squared: 0.2604

\#\# F-statistic: 25.29 on 3 and 204 DF, p-value: 5.939e-14

round (coef (dist2), 3)

\begin{tabular}{|c|c|}
\hline \#\# & distractibility.s_c \\
\hline \#\# & 0.412 \\
\hline \#\# & gen.z distractibility.s_c:gen.z \\
\hline \#\# & $0.148 \quad-0.068$ \\
\hline
\end{tabular}

round (confint (dist2), 3)

\begin{tabular}{|rrr}
$\# \#$ \# & $2.5 \div$ & $97.5 \%$ \\
$\#$ (Intercept) & 0.138 & 0.319 \\
$\# \#$ distractibility.s_c & 0.283 & 0.542 \\
$\# \#$ gen.z & 0.054 & 0.243 \\
$\#$ \# distractibility.s_c:gen.z & -0.184 & 0.048 \\
\hline
\end{tabular}

anova (dist1, dist2)

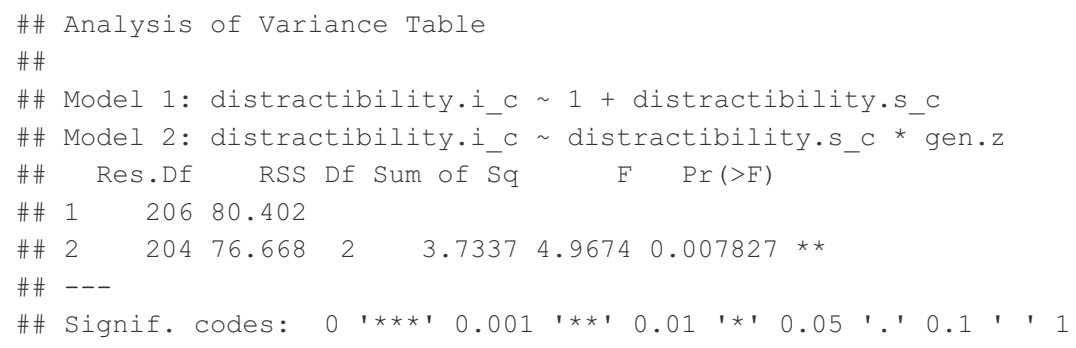

\#\# Eccentricity \#\#

t.test (pp.full\$eccentricity.s, pp.full\$eccentricity.i, paired $=\mathrm{T}$ )

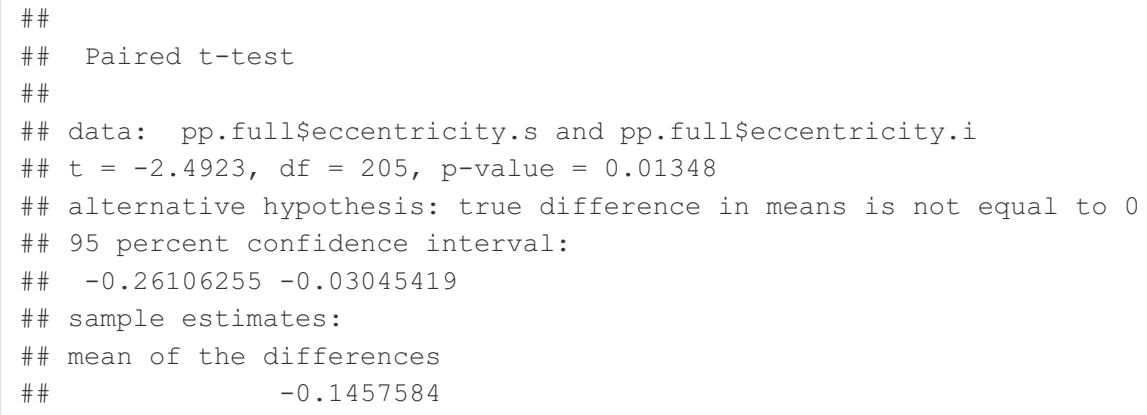


\#\#

\#\# Wilcoxon signed rank test with continuity correction

\#\#

\#\# data: pp.full\$eccentricity.s and pp.full\$eccentricity.i

\#\# $\mathrm{V}=6857, \mathrm{p}$-value $=0.02963$

\#\# alternative hypothesis: true location shift is not equal to 0

(2.4923/sqrt (206))

\#\# [1] 0.1736468

pnorm (.174)

\#\# [1] 0.5690673

$(2 * \operatorname{pnorm}((-\operatorname{abs}(.174)) / 2))$

\#\# [1] 0.9306715

print(corr.test(pp.full\$eccentricity.s, pp.full\$eccentricity.i, method = "spearman"), short = F, digits = 3)

\#\# Call:corr.test $(x=p p . f u l l \$ e c c e n t r i c i t y . s, y=p p . f u l l \$ e c c e n t r i c i t y . i$,

\#\# method = "spearman")

\#\# Correlation matrix

\#\# [1] 0.227

\#\# Sample Size

\#\# [1] 206

\#\# Probability values adjusted for multiple tests.

\#\# [1] 0.001

\#\#

\#\# Confidence intervals based upon normal theory. To get bootstrapped values, try cor.ci

\#\# raw.lower raw.r raw.upper raw.p lower.adj upper.adj

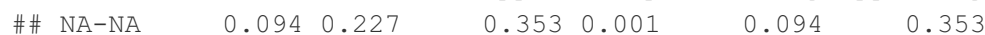

\# Base model

pp.full\$eccentricity.s_c = pp.full\$eccentricity.s - mean (pp.full\$eccentricity.s)

pp.full\$eccentricity.i_c $=$ pp.full\$eccentricity.i - mean (pp.full\$eccentricity.s)

ecc1 $=\operatorname{lm}($ eccentricity.i_c $\sim 1$ + eccentricity.s_c, data = pp.full)

summary (ecc1)

\#\#

\#\# Call:

\#\# $\operatorname{lm}$ (formula = eccentricity.i_c 1 + eccentricity.s_c, data $=$ pp.full)

\#\#

\#\# Residuals:

\# \# Min 10 Median $30 \quad$ Max

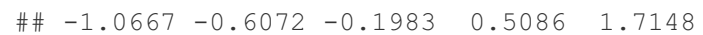

\#\#

\#\# Coefficients:

\#\# Estimate Std. Error $t$ value $\operatorname{Pr}(>|t|)$

\#\# (Intercept) $0.15007 \quad 0.04747 \quad 3.1610 .001810$ **

\#\# eccentricity.s_c $0.25798 \quad 0.07168 \quad 3.5990 .000401 * * *$

\#\#---

\#\# Signif. codes: 0 '***' 0.001 '**' 0.01 '*' 0.05 '.' 0.1 ' ' 1

\#\#

\#\# Residual standard error: 0.6813 on 204 degrees of freedom

\#\# (2 observations deleted due to missingness)

\#\# Multiple R-squared: 0.0597, Adjusted R-squared: 0.05509

\#\# F-statistic: 12.95 on 1 and 204 DF, p-value: 0.0004012 


\begin{tabular}{|lrr}
\hline$\# \#$ & $2.5 \%$ & $97.5 \%$ \\
$\# \#$ (Intercept) & 0.056 & 0.244 \\
$\# \#$ eccentricity.s_c & 0.117 & 0.399 \\
\hline
\end{tabular}

\# Moderation model

ecc2 = lm(eccentricity.i_c $\sim$ eccentricity.s_c*gen.z, data = pp.full)

summary (ecc2)

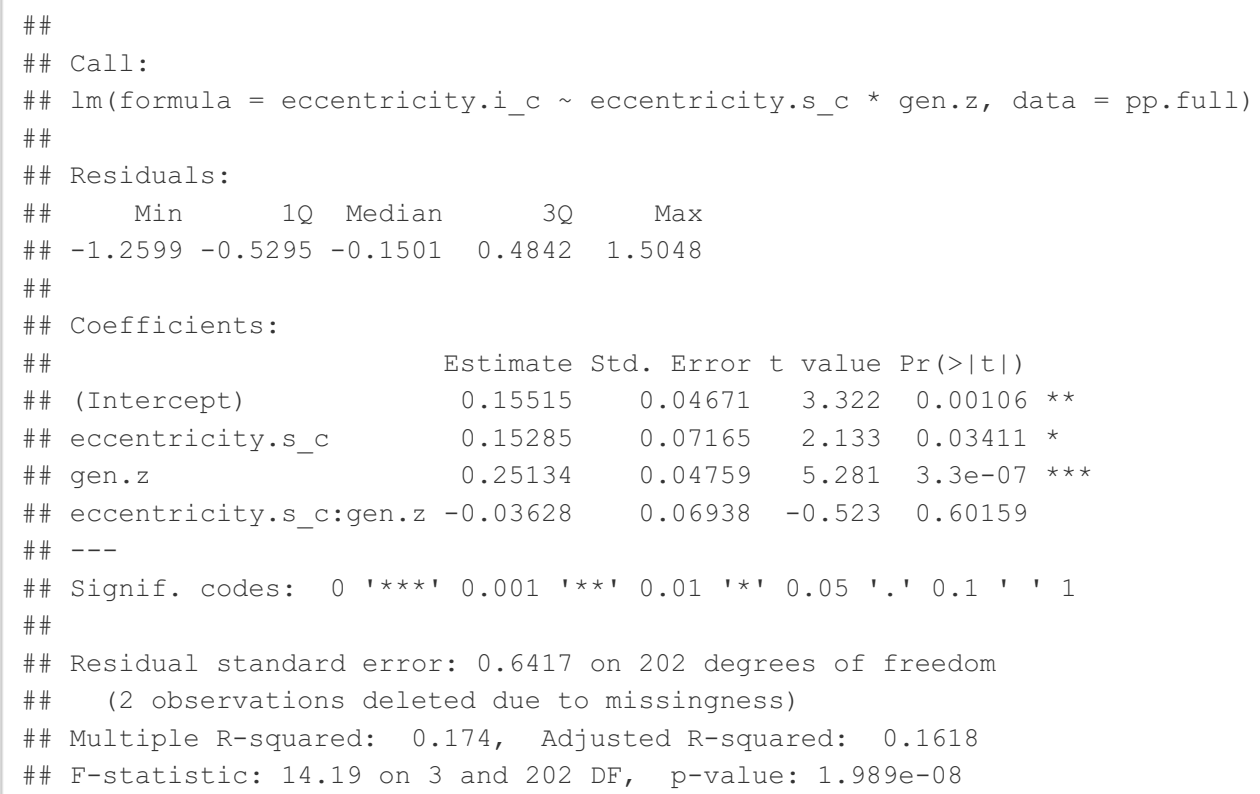

\begin{tabular}{|lrr|}
\hline$\# \#$ & $2.5 \div$ & $97.5 \%$ \\
$\#$ (Intercept) & 0.063 & 0.247 \\
$\# \#$ eccentricity.s_c & 0.012 & 0.294 \\
$\# \#$ gen.z & 0.158 & 0.345 \\
$\# \#$ eccentricity.s_c:gen.z & -0.173 & 0.101 \\
\hline
\end{tabular}

anova (ecc1, ecc2)

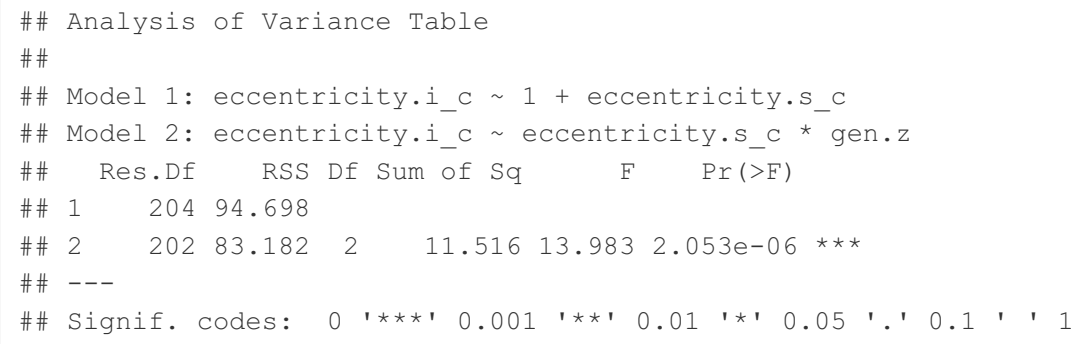




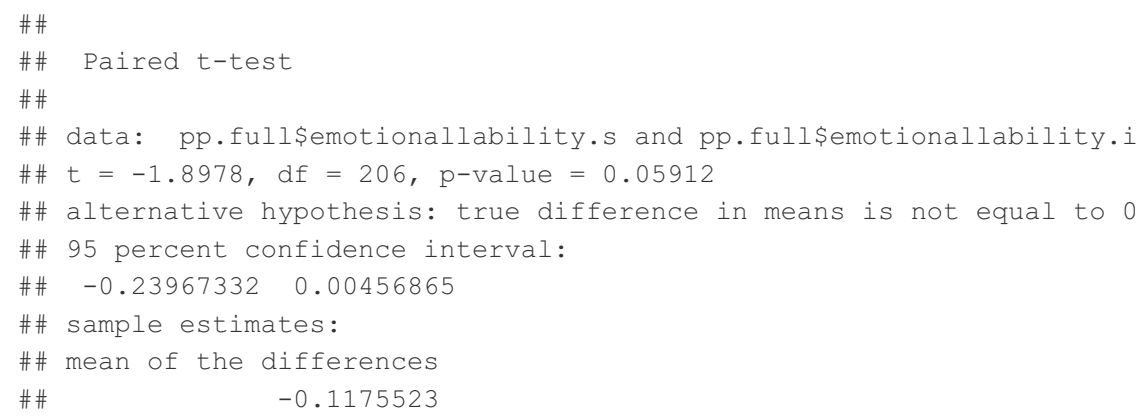

\# \# [1] 0.1319062

pnorm (.132)

\#\# [1] 0.5525079

$(2 * \operatorname{pnorm}((-\operatorname{abs}(.132)) / 2))$

\#\# [1] 0.9473778

print(corr.test(pp.full\$emotionallability.s, pp.full\$emotionallability.i, method = "spearman"), short = F, d igits $=3$ )

\#\# Call:corr.test $(x=p p . f u l l \$$ emotionallability.s, $y=p p . f u l l \$$ emotionallability.i,

\#\# $\quad$ method = "spearman")

\#\# Correlation matrix

\#\# [1] 0.343

\#\# Sample Size

\#\# [1] 207

\#\# Probability values adjusted for multiple tests.

\#\# [1] 0

\#\#

\#\# Confidence intervals based upon normal theory. To get bootstrapped values, try cor.ci

\#\# raw.lower raw.r raw.upper raw.p lower.adj upper.adj
\#\# NA-NA
0.2170 .343
0.458
0.217
0.458

\# Base model

pp.full\$emotionallability.s_c = pp.full\$emotionallability.s - mean(pp.full\$emotionallability.s)

pp.full\$emotionallability.i_c = pp.full\$emotionallability.i - mean(pp.full\$emotionallability.s)

emot1 = lm(emotionallability.i_c 1 + emotionallability.s_c, data = pp.full)

summary (emot1) 
\#\# Call:

\#\# $\operatorname{lm}($ formula = emotionallability.i_c $~ 1+$ emotionallability.s_c,

\#\# data $=$ pp.full)

\#\#

\#\# Residuals:

\#\# Min 10 Median $30 \quad$ Max

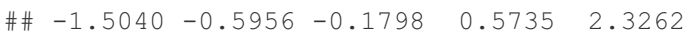

\#\#

\#\# Coefficients:

\#\# Estimate std. Error $t$ value $\operatorname{Pr}(>|t|)$

\#\# (Intercept) $0.11279 \quad 0.05322 \quad 2.119 \quad 0.0353$ *

\#\# emotionallability.s_c $0.36321 \quad 0.07398 \quad 4.9101 .86 e-06 * \star *$

\#\# ---

\#\# Signif. codes: 0 '***' 0.001 '**' 0.01 '*' 0.05 '.' 0.1 ' ' 1

\#\#

\#\# Residual standard error: 0.7656 on 205 degrees of freedom

\#\# (1 observation deleted due to missingness)

\#\# Multiple R-squared: 0.1052, Adjusted R-squared: 0.1008

\#\# F-statistic: 24.1 on 1 and 205 DF, p-value: $1.86 e-06$

round (confint (emot1), 3)

$\begin{array}{rrr}\# \# & 2.5 \div & 97.5 \% \\ \# \# \text { (Intercept) } & 0.008 & 0.218 \\ \# \# \text { emotionallability.s_c } & 0.217 & 0.509\end{array}$

\# Moderation model

emot2 = lm(emotionallability.i $c \sim$ emotionallability.s $c^{\star}$ gen.z, data = pp.full)

summary (emot2)

\#\#

\#\# Call:

\#\# Im(formula = emotionallability.i_c emotionallability.s_c *

\#\# gen.z, data $=$ pp.full)

\#\#

\#\# Residuals:

\# Min $1 Q$ Median $3 Q \quad$ Max

$\begin{array}{llllll}\# \# & -1.6976 & -0.5070 & -0.1617 & 0.4952 & 2.1684\end{array}$

\#\#

\#\# Coefficients:

\#\#

Estimate Std. Error t value $\operatorname{Pr}(>|t|)$

$\begin{array}{lllll}\text { \# } \text { gen.z } & 0.33044 & 0.05487 & 6.023 & 7.93 e-09 * * *\end{array}$

\#\# emotionallability.s c:gen.z $-0.02660 \quad 0.07166 \quad-0.371 \quad 0.7109$

\#\#---

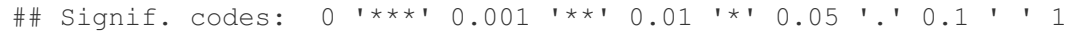

\#\#

\#\# Residual standard error: 0.7078 on 203 degrees of freedom

\#\# (1 observation deleted due to missingness)

\#\# Multiple R-squared: 0.2427, Adjusted R-squared: 0.2315

\#\# F-statistic: 21.68 on 3 and 203 DF, p-value: $3.192 e-12$

round (confint (emot2), 3)

\begin{tabular}{lrr}
\hline$\# \#$ & $2.5 \%$ & $97.5 \%$ \\
$\# \#$ (Intercept) & 0.014 & 0.225 \\
\#\# emotionallability.s_c & 0.032 & 0.330 \\
\#\# gen.z & 0.222 & 0.439 \\
\#\# emotionallability.s_c:gen.z & -0.168 & 0.115
\end{tabular}

anova (emot1, emot2) 


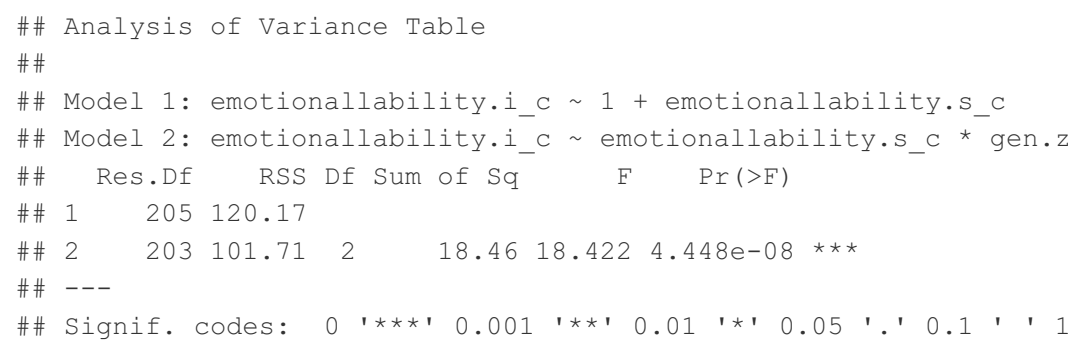

\section{\#\# Grandiosity \#\#}

t.test (pp.full\$grandiosity.s, pp.full\$grandiosity.i, paired $=\mathrm{T}$ )

\section{\#\#}

\#\# Paired t-test

\#\#

\#\# data: pp.full\$grandiosity.s and pp.full\$grandiosity.i

$\# \#=-2.6954, \mathrm{df}=207, \mathrm{p}$-value $=0.007608$

\#\# alternative hypothesis: true difference in means is not equal to 0

\#\# 95 percent confidence interval:

\#\# $-0.25555206-0.03964025$

\#\# sample estimates:

\#\# mean of the differences

\#\# -0.1475962

wilcox.test (pp.full\$grandiosity.s, pp.full\$grandiosity.i, paired $=\mathrm{T}$ )

\#\#

\#\# Wilcoxon signed rank test with continuity correction

\#\#

\#\# data: pp.full\$grandiosity.s and pp.full\$grandiosity.i

\#\# $\mathrm{V}=5991, \mathrm{p}$-value $=0.01478$

\#\# alternative hypothesis: true location shift is not equal to 0

$(2.6954 /$ sqrt (208))

\#\# [1] 0.1868924

pnorm (.187)

\#\# [1] 0.5741697

$(2 * \operatorname{pnorm}((-\operatorname{abs}(.187)) / 2))$

\#\# [1] 0.9255063

print(corr.test(pp.full\$grandiosity.s, pp.full\$grandiosity.i, method = "spearman"), short = F, digits = 3)

\#\# Call:corr.test $(\mathrm{x}=\mathrm{pp}$.full\$grandiosity.s, $\mathrm{y}=\mathrm{pp}$.full\$grandiosity.i,

\#\# method = "spearman")

\#\# Correlation matrix

\# \# [1] 0.289

\#\# Sample Size

\#\# [1] 208

\#\# Probability values adjusted for multiple tests.

\#\# [1] 0

\#\#

\#\# Confidence intervals based upon normal theory. To get bootstrapped values, try cor.ci

\#\# raw.lower raw.r raw.upper raw.p lower.adj upper.adj

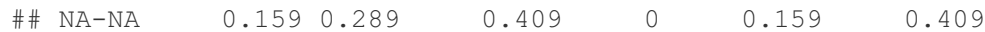


* Base model

pp.full\$grandiosity.s_c = pp.full\$grandiosity.s - mean(pp.full\$grandiosity.s)

pp.full\$grandiosity.i_c = pp.full\$grandiosity.i - mean(pp.full\$grandiosity.s)

gral = lm(grandiosity.i_c 1 +grandiosity.s_c, data $=$ pp.full)

summary (gra1)

\#\#

\#\# Call:

\#\# $\operatorname{lm}($ formula $=$ grandiosity.i_c $\sim 1$ +grandiosity.s_c, data $=$ pp.full)

\#\#

\#\# Residuals:

\#\# Min 10 Median $30 \quad$ Max

\#\# $-1.2958-0.5219-0.1469 \quad 0.4829 \quad 2.3162$

\#\#

\#\# Coefficients:

\#\# Estimate Std. Error $t$ value $\operatorname{Pr}(>|t|)$

\#\# (Intercept) $0.14760 \quad 0.04949 \quad 2.9820 .00321$ **

\#\# grandiosity.s_c $0.38932 \quad 0.08870 \quad 4.3891 .82 e-05 * * *$

\#\# ---

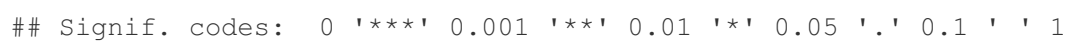

\#\#

\#\# Residual standard error: 0.7138 on 206 degrees of freedom

\#\# Multiple R-squared: 0.08553, Adjusted R-squared: 0.08109

\#\# F-statistic: 19.27 on 1 and 206 DF, p-value: $1.816 e-05$

round (confint (gra1), 3)

$\begin{array}{llrr}\text { \#\# } & & 2.5 \% & 97.5 \% \\ \text { \#\# } & \text { (Intercept) } & 0.050 & 0.245 \\ \text { \#\# grandiosity.s c } & 0.214 & 0.564\end{array}$

\# Moderation model

gra2 $=\operatorname{lm}($ grandiosity.i_c $\sim$ grandiosity.s_c*gen.z, data $=$ pp.full)

summary (gra2)

$\# \#$

\#\# Call:

\#\# $\operatorname{lm}($ formula $=$ grandiosity.i c $\sim$ grandiosity.s c $*$ gen.z, data $=$ pp.full)

\#\#

\#\# Residuals:

\#\# Min 10 Median $30 \quad$ Max

$\begin{array}{llllll}\# \# & -1.2806 & -0.4977 & -0.1599 & 0.3956 & 2.3618\end{array}$

\#\#

\#\# Coefficients:

\#\#

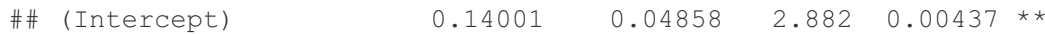

\#\# grandiosity.s c $\quad 0.26450 \quad 0.08903 \quad 2.971 \quad 0.00332 * *$

\#\# gen.z $0.23623 \quad 0.04903 \quad 4.818 \quad 2.83 e-06 * * *$

$\begin{array}{llllll}\text { \#\# grandiosity.s c:gen.z } & 0.05030 & 0.07981 & 0.630 & 0.52927\end{array}$

\#\# ---

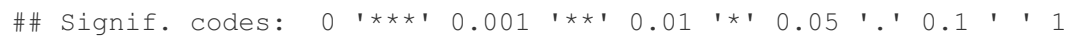

\#\#

\#\# Residual standard error: 0.6787 on 204 degrees of freedom

\#\# Multiple R-squared: 0.1812, Adjusted R-squared: 0.1691

\#\# F-statistic: 15.04 on 3 and 204 DF, p-value: 6.973e-09

round (confint (gra2), 3)

$\begin{array}{lrr}\text { \#\# } & 2.5 \% & 97.5 \% \\ \text { \#\# (Intercept) } & 0.044 & 0.236 \\ \text { \#\# grandiosity.s_c } & 0.089 & 0.440 \\ \text { \#\# gen.z } & 0.140 & 0.333 \\ \text { \#\# grandiosity.s_c:gen.z } & -0.107 & 0.208\end{array}$


\#\# Analysis of Variance Table

\#\#

\#\# Model 1: grandiosity.i_c 1 + grandiosity.s_c

\#\# Model 2: grandiosity.i_c $\sim$ grandiosity.s_c * gen.z

\#\# Res.Df RSS Df Sum of Sq F $\operatorname{Pr}(>\mathrm{F})$

$\begin{array}{llll}\# \# & 1 & 206 & 104.952\end{array}$

\#\# $2 \quad 204 \quad 93.9772 \quad 10.974 \quad 11.911 \quad 1.281 \mathrm{e}-05$ ***

\#\# ---

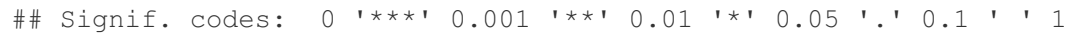

\#\# Hostility \#\#

t.test(pp.full\$hostility.s, pp.full\$hostility.i, paired $=T$ )

\#\#

\#\# Paired t-test

\#\#

\#\# data: pp.full\$hostility.s and pp.full\$hostility.i

$\# \#=-3.7197, \mathrm{df}=206, \mathrm{p}$-value $=0.0002572$

\#\# alternative hypothesis: true difference in means is not equal to 0

\#\# 95 percent confidence interval:

\#\# $-0.31555246-0.09692741$

\#\# sample estimates:

\#\# mean of the differences

\#\#

$-0.2062399$

wilcox.test (pp.full\$hostility.s, pp.full\$hostility.i, paired $=\mathrm{T}$ )

\#\#

\#\# Wilcoxon signed rank test with continuity correction

\#\#

\#\# data: pp.full\$hostility.s and pp.full\$hostility.i

\#\# $\mathrm{V}=6970.5, \mathrm{p}$-value $=0.002101$

\#\# alternative hypothesis: true location shift is not equal to 0

$(3.7197$ /sqrt (207))

\# \# [1] 0.258537

pnorm (.259)

\#\# [1] 0.6021824

$(2 * \operatorname{pnorm}((-\operatorname{abs}(.259)) / 2))$

\#\# [1] 0.896962

print(corr.test (pp.full\$hostility.s, pp.full\$hostility.i, method = "spearman"), short = F, digits = 3)

\#\# Call:corr.test ( $\mathrm{x}=$ pp.full\$hostility.s, $y=$ pp.full\$hostility.i, method = "spearman")

\#\# Correlation matrix

\#\# [1] 0.336

\#\# Sample Size

\#\# [1] 207

\#\# Probability values adjusted for multiple tests.

\#\# [1] 0

\#\#

\#\# Confidence intervals based upon normal theory. To get bootstrapped values, try cor.ci

\#\# raw.lower raw.r raw.upper raw.p lower.adj upper.adj

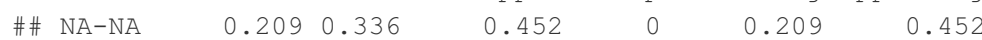


\# Base model

pp.full\$hostility.s_c = pp.full\$hostility.s - mean(pp.full\$hostility.s, na.rm = T)

pp.full\$hostility.i_c $=$ pp.full\$hostility.i - mean(pp.full\$hostility.s, na.rm = T)

hos1 = lm(hostility.i_c $\sim 1+$ hostility.s_c, data $=$ pp.full)

summary (hos 1$)$

\#\#

\# \# Call:

\#\# lm(formula = hostility.i_c 1 + hostility.s_c, data = pp.full)

\#\#

\#\# Residuals:

\#\# Min 10 Median $30 \quad$ Max

$\begin{array}{llllll}\# \# & -1.2284 & -0.5853 & -0.1445 & 0.4864 & 2.0764\end{array}$

\#\#

\#\# Coefficients:

\#\# Estimate Std. Error t value $\operatorname{Pr}(>|t|)$

\#\# (Intercept) $0.20624 \quad 0.04943 \quad 4.1724 .46 e-05 * \star *$

\#\# hostility.s_c $0.38263 \quad 0.08390 \quad 4.5618 .77$ e-06***

\#\#---

\#\# Signif. codes: 0 '***' 0.001 '**' 0.01 '*' 0.05 '.' 0.1 ' ' 1

\#\#

\#\# Residual standard error: 0.7112 on 205 degrees of freedom

\#\# (1 observation deleted due to missingness)

\#\# Multiple R-squared: 0.09211, Adjusted R-squared: 0.08768

\#\# F-statistic: 20.8 on 1 and 205 DF, p-value: $8.77 e-06$

round (confint (hos1), 3)

$\begin{array}{lrrr}\text { \#\# } & 2.5 \% & 97.5 \% \\ \# \# \text { (Intercept) } & 0.109 & 0.304 \\ \text { \#\# hostility.s_c } & 0.217 & 0.548\end{array}$

\# Moderation model

hos2 = lm(hostility.i_c $\sim$ hostility.s_c*gen.z, data $=$ pp.full)

summary (hos2)

\#\#

\#\# Call:

\#\# $\operatorname{lm}($ formula $=$ hostility.i c $\sim$ hostility.s $c *$ gen.z, data $=$ pp.full)

\#\#

\#\# Residuals:

\#\# $\quad$ Min $\quad 10$ Median $30 \quad$ Max

$\begin{array}{llllll}\# \#-1.16947 & -0.47734 & -0.09274 & 0.40610 & 2.04132\end{array}$

\#\#

\#\# Coefficients:

\#\#

\#\# (Intercept)

\#\# hostility.s_c

Std. Error $t$ value $\operatorname{Pr}(>|t|)$

$\begin{array}{llll}-0.069493 & 0.097300 & -0.714 & 0.476\end{array}$

\#\# gen.z $\quad 0.456962 \quad 0.054595 \quad 8.370 \quad 9.30$ e-15***

$\begin{array}{llllll}\text { \#\# hostility.s_c:gen.z } & -0.006877 & 0.060940 & -0.113 & 0.910\end{array}$

$\# \#---$

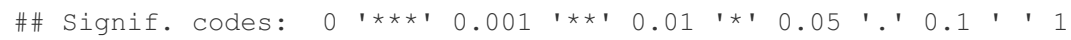

\#\#

\#\# Residual standard error: 0.6162 on 203 degrees of freedom

\#\# (1 observation deleted due to missingness)

\#\# Multiple R-squared: 0.3252, Adjusted R-squared: 0.3152

\#\# F-statistic: 32.6 on 3 and 203 DF, p-value: < 2.2e-16

round (confint (hos2), 3)

$\begin{array}{rrr}\text { \#\# } & 2.5 \% & 97.5 \% \\ \# \# \text { (Intercept) } & 0.121 & 0.309 \\ \text { \#\# hostility.s_c } & -0.261 & 0.122 \\ \text { \#\# gen.z } & 0.349 & 0.565 \\ \text { \#\# hostility.s_c:gen.z } & -0.127 & 0.113\end{array}$


\#\# Analysis of Variance Table

\#\#

\#\# Model 1: hostility.i_c 1 + hostility.s_c

\#\# Model 2: hostility.i_c $\sim$ hostility.s_c * gen.z

\#\# Res.Df RSS Df Sum of Sq F $\operatorname{Pr}(>\mathrm{F})$

$\begin{array}{llll}\# \# & 1 & 205 & 103.698\end{array}$

\#\# $2203 \quad 77.0792 \quad 26.619 \quad 35.052 \quad 8.384 e_{-14}$ ***

\#\# ---

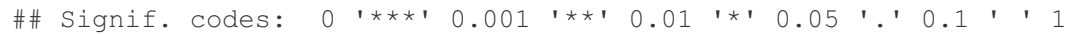

\#\# Impulsivity \#\#

t.test (pp.full\$impulsivity.s, pp.full\$impulsivity.i, paired $=\mathrm{T}$ )

\#\#

\#\# Paired t-test

\#\#

\#\# data: pp.full\$impulsivity.s and pp.full\$impulsivity.i

\#\# $t=-5.013, \mathrm{df}=205, \mathrm{p}$-value $=1.157 \mathrm{e}-06$

\#\# alternative hypothesis: true difference in means is not equal to 0

\#\# 95 percent confidence interval:

\#\# $-0.3632047-0.1581545$

\#\# sample estimates:

\#\# mean of the differences

\#\#

$-0.2606796$

wilcox.test (pp.full\$impulsivity.s, pp.full\$impulsivity.i, paired $=\mathrm{T}$ )

\#\#

\#\# Wilcoxon signed rank test with continuity correction

\#\#

\#\# data: pp.full\$impulsivity.s and pp.full\$impulsivity.i

\#\# $\mathrm{V}=5444, \mathrm{p}$-value $=2.676 \mathrm{e}-06$

\#\# alternative hypothesis: true location shift is not equal to 0

$(5.013 / \operatorname{sqrt}(206))$

\# [1] 0.3492723

$\operatorname{pnorm}(.349)$

\#\# [1] 0.6364553

$(2 * \operatorname{pnorm}((-\operatorname{abs}(.349)) / 2))$

\#\# [1] 0.8614725

print(corr.test(pp.full\$impulsivity.s, pp.full\$impulsivity.i, method = "spearman"), short = F, digits = 3) 
\#\# Call:corr.test $(x=p p . f u l l \$ i m p u l s i v i t y . s, y=p p . f u l l \$ i m p u l s i v i t y \cdot i$,

\#\# $\quad$ method = "spearman")

\#\# Correlation matrix

\#\# [1] 0.495

\#\# Sample Size

\#\# [1] 206

\#\# Probability values adjusted for multiple tests.

\#\# [1] 0

\#\#

\#\# Confidence intervals based upon normal theory. To get bootstrapped values, try cor.ci

\#\# raw.lower raw.r raw.upper raw.p lower.adj upper.adj

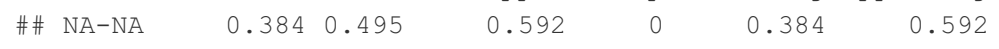

\section{\# Base model}

pp.full\$impulsivity.s_c $=$ pp.full\$impulsivity.s - mean(pp.full\$impulsivity.s)

pp.full\$impulsivity.i_c $=$ pp.full\$impulsivity.i - mean(pp.full\$impulsivity.s)

im1 $=$ lm(impulsivity.i_c $\sim 1+$ impulsivity.s_c, data $=$ pp.full)

summary (im1)

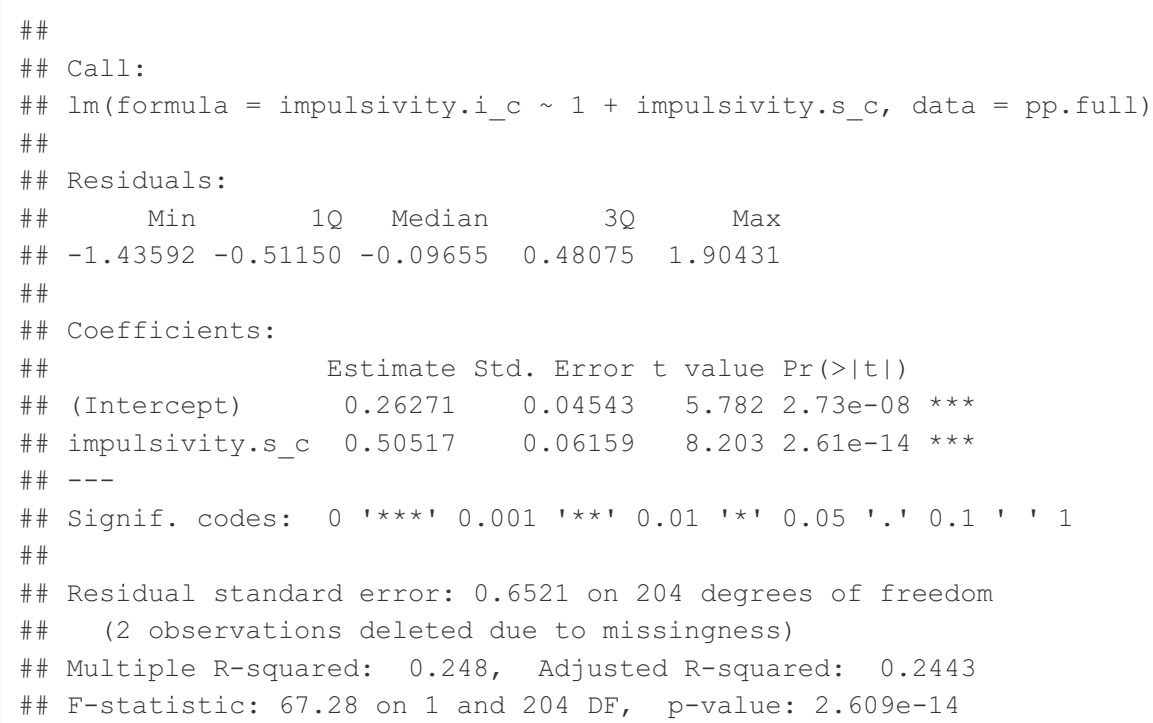

round (confint (im1), 3)

\begin{tabular}{llrr}
\hline$\# \#$ & $2.5 \%$ & $97.5 \%$ \\
$\# \#$ (Intercept) & 0.173 & 0.352 \\
\#\# impulsivity.s_c & 0.384 & 0.627
\end{tabular}

\# Moderation model

im2 = lm(impulsivity.i_c $\sim$ impulsivity.s_c*gen.z, data = pp.full) summary (im2) 
\#\# Call:

\#\# $\operatorname{lm}$ (formula = impulsivity.i c $\sim$ impulsivity.s $c$ * gen.z, data $=$ pp.full)

\#\#

\#\# Residuals:

\#\# Min $\quad 10 \quad$ Median $\quad 30 \quad$ Max

$\begin{array}{llllll}\# \# & -1.56847 & -0.46728 & -0.05301 & 0.43632 & 1.73947\end{array}$

\#\#

\#\# Coefficients:

\#\# Estimate Std. Error $t$ value Pr $(>|t|)$

\#\# (Intercept) $0.27700 \quad 0.04587 \quad 6.038 \quad 7.35 e-09 * * *$

\#\# impulsivity.s_c $\quad 0.47053 \quad 0.06377 \quad 7.3794 .07$ e-12 $* * *$

$\begin{array}{llllll}\text { \# } \text { gen.z } & 0.14173 & 0.04663 & 3.039 & 0.00268 * *\end{array}$

$\begin{array}{llllll}\text { \# impulsivity.s_c:gen.z } & -0.07298 & 0.05448 & -1.340 & 0.18190\end{array}$

\#\#---

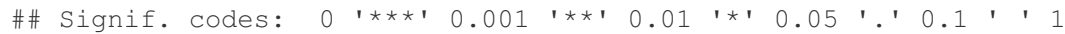

\#\#

\#\# Residual standard error: 0.6394 on 202 degrees of freedom

\#\# (2 observations deleted due to missingness)

\#\# Multiple R-squared: 0.284, Adjusted R-squared: 0.2734

\#\# F-statistic: 26.71 on 3 and 202 DF, p-value: 1.357e-14

round (confint (im2), 3)

$\begin{array}{lrr}\text { \#\# } & 2.5 \% & 97.5 \% \\ \# \# \text { (Intercept) } & 0.187 & 0.367 \\ \text { \#\# impulsivity.s_c } & 0.345 & 0.596 \\ \text { \#\# gen.z } & 0.050 & 0.234 \\ \text { \#\# impulsivity.s_c:gen.z } & -0.180 & 0.034\end{array}$

anova (im1, im2)

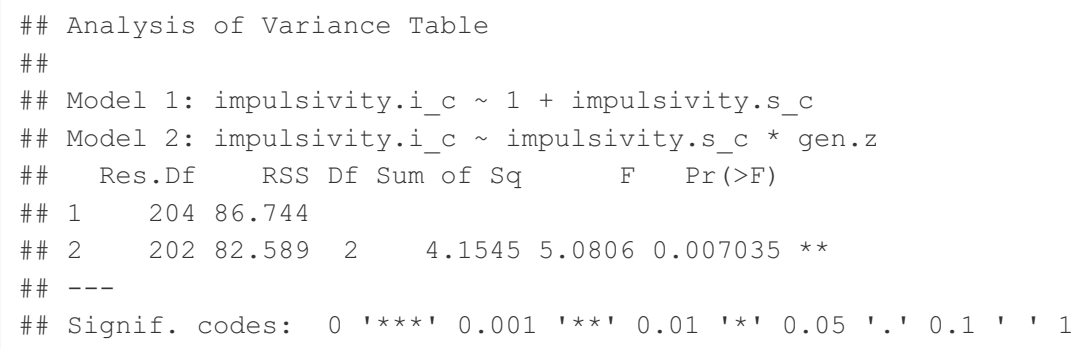

wilcox.test (pp.full\$intimacyavoidance.s, pp.full\$intimacyavoidance.i, paired = T) 
\#\# [1] 0.2227852

$\operatorname{pnorm}(.223)$

\#\# [1] 0.5882322

$(2 * \operatorname{pnorm}((-\operatorname{abs}(.223)) / 2))$

\#\# [1] 0.9112199

print (corr.test (pp.full\$intimacyavoidance.s, pp.full\$intimacyavoidance.i, method = "spearman"), short $=\mathrm{F}$, $\mathrm{d}$ igits $=3$ )

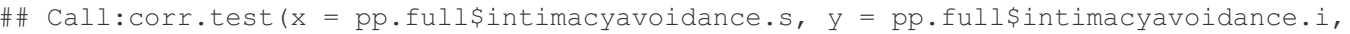

\#\# $\quad$ method = "spearman")

\#\# Correlation matrix

\# \# [1] 0.262

\#\# Sample Size

\#\# [1] 203

\#\# Probability values adjusted for multiple tests.

\#\# [1] 0

$\# \#$

\#\# Confidence intervals based upon normal theory. To get bootstrapped values, try cor.ci

\#\# raw.lower raw.r raw.upper raw.p lower.adj upper.adj
\#\# NA-NA
$0.128 \quad 0.262$
$0.385 \quad 0$
0.128
0.385

\# Base model

pp.full\$intimacyavoidance.s_c = pp.full\$intimacyavoidance.s - mean (pp.full\$intimacyavoidance.s, na.rm = T)

pp.full\$intimacyavoidance.i_c = pp.full\$intimacyavoidance.i - mean(pp.full\$intimacyavoidance.s, na.rm = T)

int $1=\operatorname{lm}($ intimacyavoidance.i $\mathrm{c} \sim 1$ + intimacyavoidance.s $\mathrm{c}$, data $=$ pp.full)

summary (int1)

\#\#

\# Call:

\#\# Im(formula = intimacyavoidance.i c 1 + intimacyavoidance.s c,

\#\# data $=$ pp.full

\#\#

\#\# Residuals:

\#\# Min $1 Q \quad$ Median $3 Q \quad$ Max

$\begin{array}{llllll}\text { \#\# } & -1.25120 & -0.51864 & -0.07969 & 0.37913 & 2.29822\end{array}$

\#\#

\#\# Coefficients:

\#\# Estimate Std. Error $t$ value $\operatorname{Pr}(>|t|)$

\#\# (Intercept) $0.15698 \quad 0.04249 \quad 3.6950 .000283 * \star *$

\#\# intimacyavoidance.s_c $0.36628 \quad 0.06902 \quad 5.307 \quad 2.94 e-07 * * *$

\#\# ---

\#\# Signif. codes: 0 '***' 0.001 '**' 0.01 '*' 0.05 '.'0.1 ' ' 1

\#\#

\#\# Residual standard error: 0.6053 on 201 degrees of freedom

\#\# (5 observations deleted due to missingness)

\#\# Multiple R-squared: 0.1229, Adjusted R-squared: 0.1185

\#\# F-statistic: 28.16 on 1 and 201 DF, p-value: 2.938e-07

round (confint (int1), 3)

$\begin{array}{rrr}\text { \#\# } & 2.5 \% & 97.5 \% \\ \# \# \text { (Intercept) } & 0.073 & 0.241 \\ \text { \#\# intimacyavoidance.s_c } & 0.230 & 0.502\end{array}$


\# Moderation model

int2 = lm(intimacyavoidance.i_c intimacyavoidance.s_c*gen. $z$, data $=$ pp.full)

summary (int2)

\section{\#\#}

\#\# Call:

\#\# $\operatorname{lm}($ formula = intimacyavoidance.i_c $\sim$ intimacyavoidance.s_c *

\#\# gen.z, data $=$ pp.full)

\#\#

\#\# Residuals:

\#\# Min $1 Q$ Median $3 Q \quad$ Max

$\begin{array}{llllll}\# \# & -1.3089 & -0.4422 & -0.1005 & 0.4351 & 2.2270\end{array}$

\#\#

\#\# Coefficients:

\#\#

\#\# (Intercept)

\#\# intimacyavoidance.s_c $\quad 0.24514 \quad 0.07644 \quad 3.2070 .001564$ **

\#\# gen.z $\quad 0.18465 \quad 0.04622 \quad 3.995 \quad 9.1$ e-0

$\begin{array}{llllll}\text { \# intimacyavoidance.s_c:gen.z } & 0.02563 & 0.05575 & 0.460 & 0.646243\end{array}$

\#\#---

\#\# Signif. codes: 0 '***' 0.001 '**' 0.01 '*' 0.05 '.' 0.1 ' ' 1

\#\#

\#\# Residual standard error: 0.5843 on 199 degrees of freedom

\#\# (5 observations deleted due to missingness)

\#\# Multiple R-squared: 0.1908, Adjusted R-squared: 0.1786

\#\# F-statistic: 15.64 on 3 and 199 DF, p-value: $3.586 e-09$

round (confint (int2), 3)

$\begin{array}{rrrr}\# \# & 2.5 \% & 97.5 \% \\ \# \# \text { (Intercept) } & 0.064 & 0.233 \\ \# \# \text { intimacyavoidance.s_c } & 0.094 & 0.396 \\ \# \# \text { gen.z } & 0.094 & 0.276 \\ \text { \#\# intimacyavoidance.s_c:gen.z } & -0.084 & 0.136\end{array}$

anova (int1, int2)

\#\# Analysis of Variance Table

\#\#

\#\# Model 1: intimacyavoidance.i_c 1 + intimacyavoidance.s_c

\#\# Model 2: intimacyavoidance.i_c $\sim$ intimacyavoidance.s_c * gen.z

\#\# Res.Df RSS Df Sum of Sq F Pr $(>\mathrm{F})$

\#\# $1201 \quad 73.644$

$\begin{array}{llllllll}\# 2 & 2 & 199 & 67.943 & 2 & 5.7009 & 8.3487 & 0.0003299\end{array} * *$

\#\#---

\#\# Signif. codes: 0 '***' 0.001 '**' 0.01 '*' 0.05 '.'0.1 ' ' 1

\#\# Irresponsibility \#\#

t.test(pp.full\$irresponsibility.s, pp.full\$irresponsibility.i, paired = T)

\#\#

\#\# Paired t-test

\#\#

\#\# data: pp.full\$irresponsibility.s and pp.full\$irresponsibility.i

\#\# $t=-5.8829, \mathrm{df}=206, \mathrm{p}$-value $=1.611 \mathrm{e}-08$

\#\# alternative hypothesis: true difference in means is not equal to 0

\#\# 95 percent confidence interval:

\#\# $-0.3203450-0.1595261$

\#\# sample estimates:

\#\# mean of the differences

\#\# $\quad-0.2399356$

wilcox.test (pp.full\$irresponsibility.s, pp.full\$irresponsibility.i, paired = T) 
\#\# Wilcoxon signed rank test with continuity correction

\#\# data: pp.full\$irresponsibility.s and pp.full\$irresponsibility.i

\#\# $\mathrm{V}=4160.5, \mathrm{p}$-value $=8.165 \mathrm{e}-08$

\#\# alternative hypothesis: true location shift is not equal to 0

\section{$(5.8829 /$ sqrt (207))}

\#\# [1] 0.4088898

$\operatorname{pnorm}(.409)$

\#\# [1] 0.6587302

$(2 * \operatorname{pnorm}((-\operatorname{abs}(.409)) / 2))$

\#\# [1] 0.8379628

print(corr.test(pp.full\$irresponsibility.s, pp.full\$irresponsibility.i, method = "spearman"), short = F, dig its $=3$ )

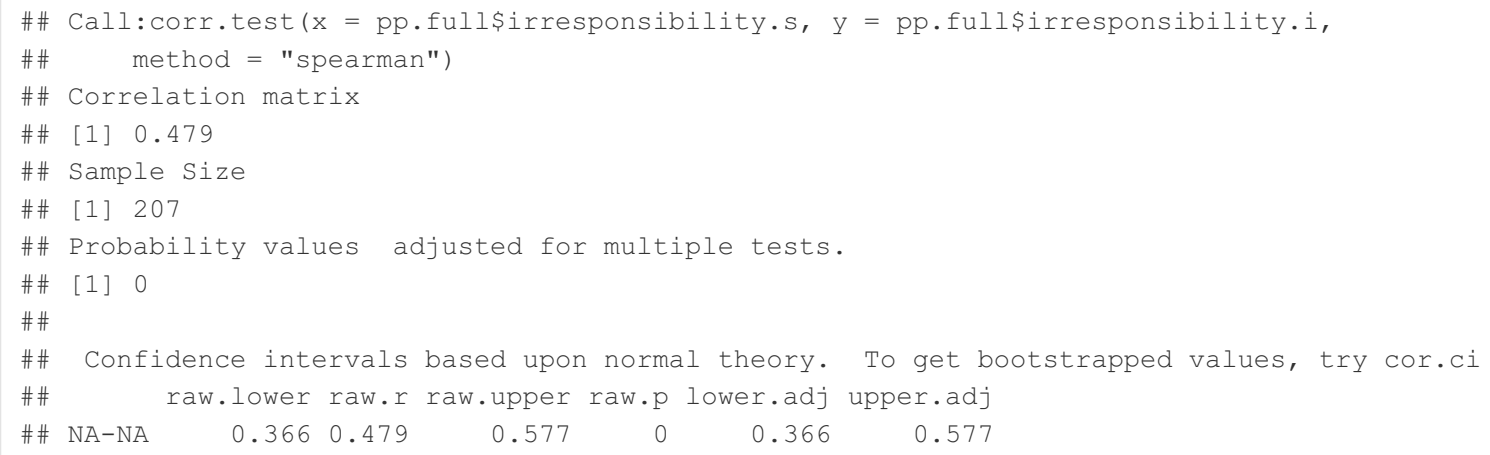




$\begin{array}{lrr}\# \# & 2.5 \% & 97.5 \% \\ \# \# \text { (Intercept) } & 0.165 & 0.314 \\ \# \# \text { irresponsibility.s_c } & 0.448 & 0.726\end{array}$

\# Moderation model

ir2 $=\operatorname{lm}($ irresponsibility.i_c $\sim$ irresponsibility.s_c*gen.z, data $=$ pp.full)

summary (ir2)

\#\#

\#\# Call:

\#\# $\operatorname{lm}($ formula $=$ irresponsibility.i_c $\sim$ irresponsibility.s_c * gen.z,

\#\# data $=$ pp.full)

\#\#

\#\# Residuals:

\#\# Min $\quad 10 \quad$ Median $30 \quad$ Max

$\begin{array}{llllll}\text { \#\# } & -1.06810 & -0.40146 & -0.01102 & 0.33321 & 1.32881\end{array}$

\#\#

\#\# Coefficients:

\#\# Estimate Std. Error $t$ value $\operatorname{Pr}(>|t|)$

\#\# (Intercept) $\quad 0.25399 \quad 0.03902 \quad 6.5105 .79 \mathrm{e}-10 * * *$

\#\# irresponsibility.s_c $\quad 0.49682 \quad 0.08053 \quad 6.169 \quad 3.66 \mathrm{e}-09 * * *$

\#\# gen.z $\quad 0.16252 \quad 0.04073 \quad 3.990 \quad 9.24 \mathrm{e}-05 * * *$

\#\# irresponsibility.s_c:gen.z $-0.07095 \quad 0.06013-1.180 \quad 0.239$

\#\# ---

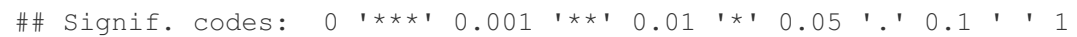

\#\#

\#\# Residual standard error: 0.5256 on 203 degrees of freedom

\#\# (1 observation deleted due to missingness)

\#\# Multiple R-squared: 0.3107, Adjusted R-squared: 0.3005

\#\# F-statistic: 30.5 on 3 and 203 DF, p-value: $2.539 e-16$

round (confint (ir2), 3)

\begin{tabular}{|lrr}
$\# \#$ & $2.5 \%$ & $97.5 \%$ \\
$\# \#$ (Intercept) & 0.177 & 0.331 \\
\#\# irresponsibility.s_c & 0.338 & 0.656 \\
\#\# gen.z & 0.082 & 0.243 \\
$\# \#$ irresponsibility.s_c:gen.z & -0.190 & 0.048
\end{tabular}

anova (ir1, ir2)

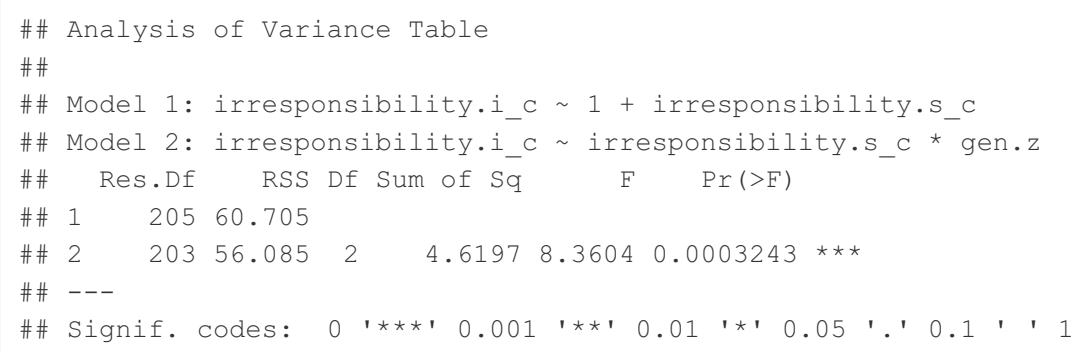

\#\# Manipulativeness \#\#

t.test (pp.full\$manipulativeness.s, pp.full\$manipulativeness.i, paired = T)

\#\#

\#\# Paired t-test

\#\#

\#\# data: pp.full\$manipulativeness.s and pp.full\$manipulativeness.i

\#\# $t=-3.3291, \mathrm{df}=207, \mathrm{p}$-value $=0.001031$

\#\# alternative hypothesis: true difference in means is not equal to 0

\#\# 95 percent confidence interval:

\#\# $\quad-0.34063790-0.08724672$

\#\# sample estimates:

\#\# mean of the differences

\#\#

$-0.2139423$ 
\#\#

\#\# Wilcoxon signed rank test with continuity correction

\#\#

\#\# data: pp.full\$manipulativeness.s and pp.full\$manipulativeness.i

\#\# $\mathrm{V}=6565$, p-value $=0.003751$

\#\# alternative hypothesis: true location shift is not equal to 0

(3.3291/sqrt (208))

\# [1] 0.2308316

pnorm (.231)

\#\# [1] 0.5913426

$(2 * \operatorname{pnorm}((-\operatorname{abs}(.231)) / 2))$

\#\# [1] 0.9080488

print(corr.test(pp.full\$manipulativeness.s, pp.full\$manipulativeness.i, method = "spearman"), short = F, dig its $=3)$

\#\# Call:corr.test $(\mathrm{x}=$ pp.full\$manipulativeness.s, $\mathrm{y}=$ pp.full\$manipulativeness.i,

\#\# $\quad$ method = "spearman")

\#\# Correlation matrix

\#\# [1] 0.198

\#\# Sample Size

\#\# [1] 208

\#\# Probability values adjusted for multiple tests.

\#\# [1] 0.004

\#\#

\# Confidence intervals based upon normal theory. To get bootstrapped values, try cor.ci

\#\# raw.lower raw.r raw.upper raw.p lower.adj upper.adj

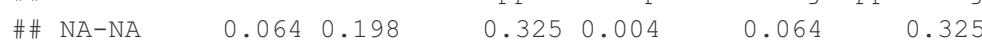

\# Base model

pp.full\$manipulativeness.s c = pp.full\$manipulativeness.s - mean(pp.full\$manipulativeness.s)

pp.full\$manipulativeness.i_c $=$ pp.full\$manipulativeness.i - mean(pp.full\$manipulativeness.s)

manil = lm(manipulativeness.i $c \sim 1+$ manipulativeness.s c, data $=$ pp.full)

summary (mani1)

\#\#

\#\# Call:

\#\# lm(formula = manipulativeness.i c 1 + manipulativeness.s_c,

\#\# data $=$ pp.full)

\#\#

\#\# Residuals:

\#\# Min 10 Median $30 \quad$ Max

$\begin{array}{llllll}\# \# & -1.3723 & -0.6447 & -0.1085 & 0.6139 & 1.9553\end{array}$

\#\#

\#\# Coefficients:

\#\# Estimate Std. Error $t$ value $\operatorname{Pr}(>|t|)$

\#\# (Intercept) $\quad 0.21394 \quad 0.05479 \quad 3.9050 .000128 * * *$

\#\# manipulativeness.s_c $0.29315 \quad 0.07963 \quad 3.6810 .000296 * * *$

\#\# ---

\#\# Signif. codes: 0 '***' 0.001 '**' 0.01 '*' 0.05 '.' 0.1 ' ' 1

\#\#

\#\# Residual standard error: 0.7902 on 206 degrees of freedom

\#\# Multiple R-squared: 0.06173, Adjusted R-squared: 0.05717

\#\# F-statistic: 13.55 on 1 and 206 DF, p-value: 0.0002963 


\begin{tabular}{|rrr}
$\# \#$ & $2.5 \div$ & $97.5 \%$ \\
$\# \#$ (Intercept) & 0.106 & 0.322 \\
$\# \#$ manipulativeness.s_c & 0.136 & 0.450 \\
\hline
\end{tabular}

\# Moderation model

mani2 = lm(manipulativeness.i_c $\sim$ manipulativeness.s_c*gen.z, data $=$ pp.full)

summary (mani2)

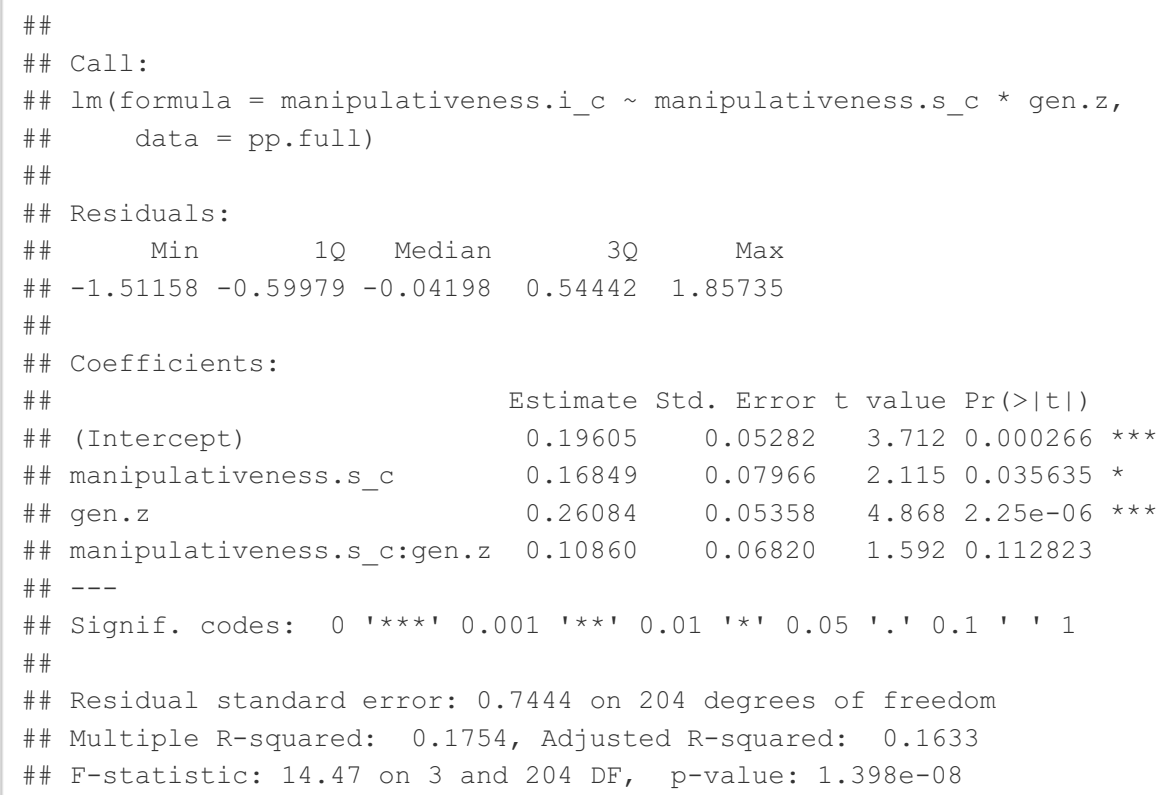

\begin{tabular}{lrr}
\hline$\# \#$ & $2.5 \div$ & $97.5 \%$ \\
$\# \#$ (Intercept) & 0.092 & 0.300 \\
\#\# manipulativeness.s_c & 0.011 & 0.326 \\
\#\# gen.z & 0.155 & 0.366 \\
\#\# manipulativeness.s_c:gen.z & -0.026 & 0.243 \\
\hline
\end{tabular}

anova (mani1, mani2)

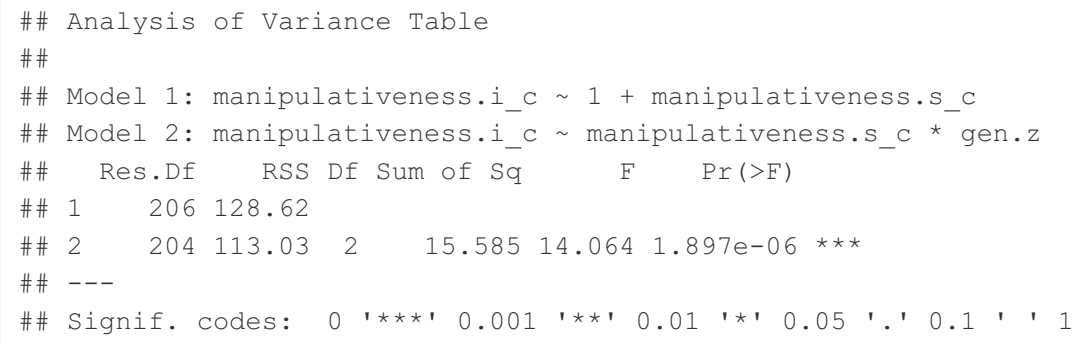




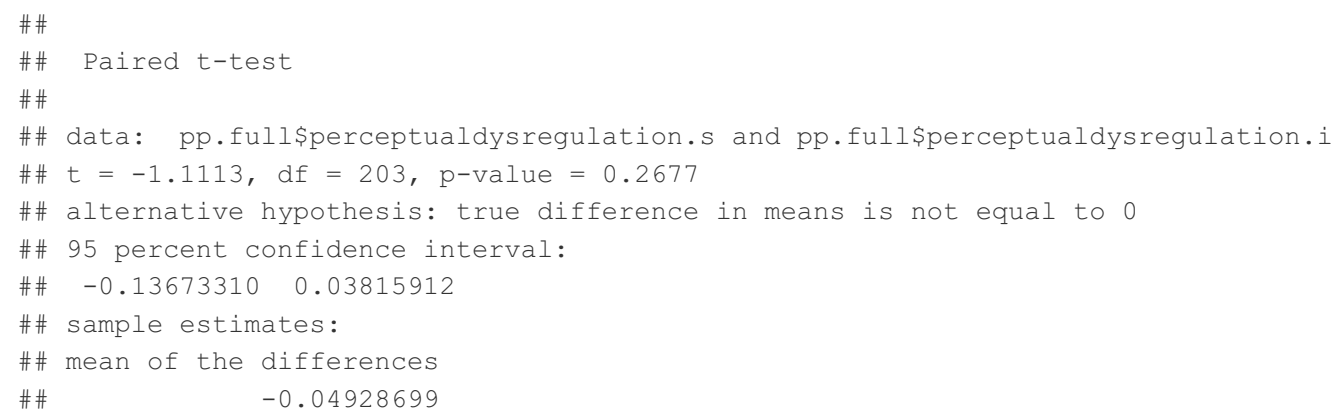

\#\# [1] 0.07780656

pnorm $(.078)$

\# \# [1] 0.531086

$(2 * \operatorname{pnorm}((-\operatorname{abs}(.078)) / 2))$

\#\# [1] 0.9688904

print (corr.test (pp.full\$perceptualdysregulation.s, pp.full\$perceptualdysregulation.i, method = "spearman"), short $=\mathrm{F}$, digits $=3$ )

\#\# Call:corr.test $(x=$ pp.full\$perceptualdysregulation.s, $y=$ pp.full\$perceptualdysregulation.i,

\#\# method = "spearman")

\#\# Correlation matrix

\#\# [1] 0.307

\#\# Sample Size

\#\# [1] 204

\#\# Probability values adjusted for multiple tests.

\#\# [1] 0

\#\#

\#\# Confidence intervals based upon normal theory. To get bootstrapped values, try cor.ci

\#\# raw.lower raw.r raw.upper raw.p lower.adj upper.adj
\#\# NA-NA
0.1770 .307
0.426
0.177
0.426

\# Base model

pp.full\$perceptualdysregulation.s_c = pp.full\$perceptualdysregulation.s - mean(pp.full\$perceptualdysregulati on.s)

pp.full\$perceptualdysregulation.i c = pp.full\$perceptualdysregulation.i - mean(pp.full\$perceptualdysregulati on.s)

per1 = lm(perceptualdysregulation.i_c $\sim 1+$ perceptualdysregulation.s_c, data = pp.full) summary (per1) 


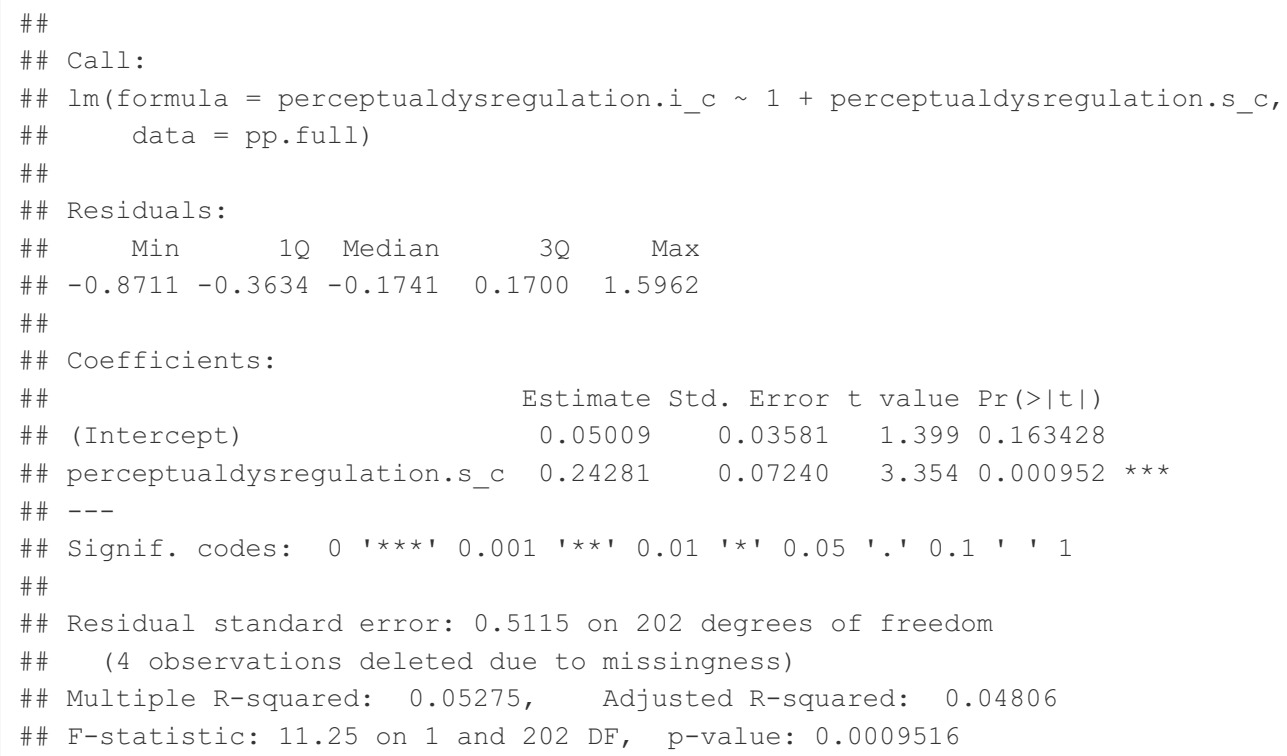

$\begin{array}{lrr}\# \# & 2.5 \% & 97.5 \% \\ \# \# \text { (Intercept) } & -0.021 & 0.121 \\ \text { \#\# perceptualdysregulation.s_c } & 0.100 & 0.386\end{array}$

\# Moderation model

per2 = lm(perceptualdysregulation.i_c $\sim$ perceptualdysregulation.s_ ${ }^{\star}$ gen.z, data $=$ pp.full) summary (per2)

\#\#

\#\# Call:

\#\# Im(formula = perceptualdysregulation.i_c perceptualdysregulation.s_c *

\#\# gen.z, data $=$ pp.full)

\#\#

\#\# Residuals:

\#\# Min $1 Q$ Median $3 Q \quad$ Max

$\begin{array}{llllll}\# \# & -0.7794 & -0.3050 & -0.1467 & 0.1730 & 1.6453\end{array}$

\#\#

\#\# Coefficients:

\#\#

\#\# (Intercept)

Estimate std. Error $t$ value $\operatorname{Pr}(>|t|)$

$0.06399-0.03577 \quad 1.789-0.0751$

\#\# perceptualdysregulation.s_c $\quad \begin{array}{lllll} & 0.17855 & 0.07853 & 2.274 & 0.0240\end{array}$ *

$\begin{array}{llllll}\text { \# } \text { gen.z } & 0.17468 & 0.03650 & 4.786 & 3.3 e-06 * * *\end{array}$

\#\# perceptualdysregulation.s_c:gen.z $-0.10073 \quad 0.07563 \quad-1.332 \quad 0.1844$

\#\#---

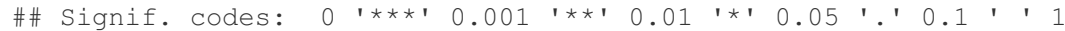

\#\#

\#\# Residual standard error: 0.4852 on 200 degrees of freedom

\#\# (4 observations deleted due to missingness)

\#\# Multiple R-squared: 0.1561, Adjusted R-squared: 0.1434

\#\# F-statistic: 12.33 on 3 and 200 DF, p-value: $1.963 e-07$

round (confint (per2), 3)

$\begin{array}{lrr}\text { \#\# } & 2.5 \% & 97.5 \% \\ \text { \#\# (Intercept) } & -0.007 & 0.135 \\ \text { \#\# perceptualdysregulation.s_c } & 0.024 & 0.333 \\ \text { \#\# gen.z } & 0.103 & 0.247 \\ \text { \#\# perceptualdysregulation.s_c:gen.z } & -0.250 & 0.048\end{array}$

anova (per1, per2) 


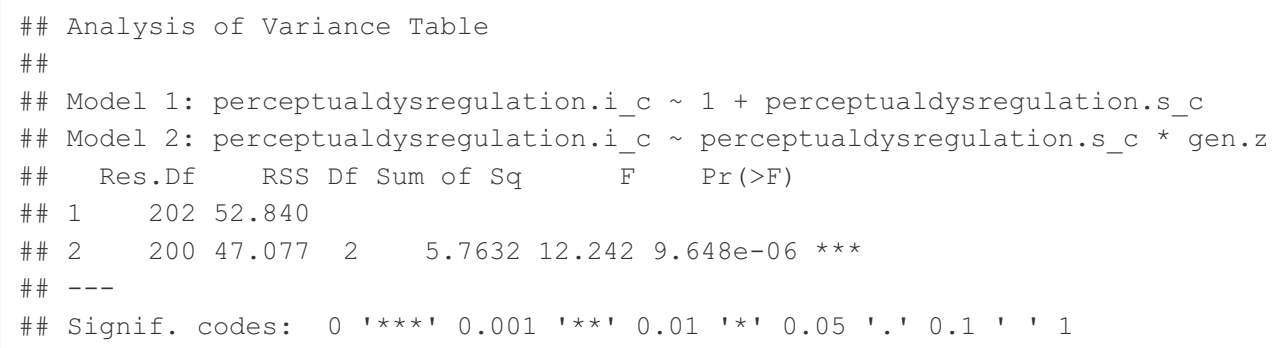

wilcox.test (pp.full\$perseveration.s, pp.full\$perseveration.i, paired $=T$ )

\#\#

\#\# Wilcoxon signed rank test with continuity correction

\#\#

\#\# data: pp.full\$perseveration.s and pp.full\$perseveration.i

\#\# $\mathrm{V}=6724.5, \mathrm{p}$-value $=0.001401$

\#\# alternative hypothesis: true location shift is not equal to 0

(3.382/sqrt (208))

\#\# [1] 0.2344995

pnorm (.234)

\#\# [1] 0.5925075

$(2 * \operatorname{pnorm}((-\operatorname{abs}(.234)) / 2))$

\#\# [1] 0.9068601

print(corr.test(pp.full\$perseveration.s, pp.full\$perseveration.i, method = "spearman"), short = F, digits = 3)

\#\# Call:corr.test $(x=$ pp.full\$perseveration.s, $y=$ pp.full\$perseveration.i,

\#\# method = "spearman")

\#\# Correlation matrix

\#\# [1] 0.433

\#\# Sample Size

\#\# [1] 208

\#\# Probability values adjusted for multiple tests.

\#\# [1] 0

\#\#

\#\# Confidence intervals based upon normal theory. To get bootstrapped values, try cor.ci

\#\# raw.lower raw.r raw.upper raw.p lower.adj upper.adj

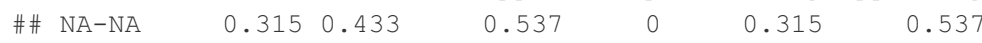


\# Base model

pp.full\$perseveration.s_c = pp.full\$perseveration.s - mean(pp.full\$perseveration.s)

pp.full\$perseveration.i_c $=$ pp.full\$perseveration.i - mean (pp.full\$perseveration.s)

pers1 = lm(perseveration.i_c 1 + perseveration.s_c, data $=$ pp.full)

summary (pers1)

\#\#

\# \# Call:

\#\# lm(formula = perseveration.i_c 1 + perseveration.s_c, data $=$ pp.full)

\#\#

\#\# Residuals:

\#\# Min $\quad$ 1Q Median $\quad 3 Q \quad$ Max

\#\# $-1.43072-0.49192 \quad-0.02761 \quad 0.41696 \quad 1.53814$

\#\#

\#\# Coefficients:

\#\# Estimate Std. Error $t$ value $\operatorname{Pr}(>|t|)$

\#\# (Intercept) $0.15834 \quad 0.04048 \quad 3.9120 .000124 * * *$

\#\# perseveration.s_c $0.45410 \quad 0.06481 \quad 7.0063 .41 e-11 * * *$

\#\#---

\#\# Signif. codes: 0 '***' 0.001 '**' 0.01 '*' 0.05 '.' 0.1 ' ' 1

\#\#

\#\# Residual standard error: 0.5838 on 206 degrees of freedom

\#\# Multiple R-squared: 0.1924, Adjusted R-squared: 0.1885

\#\# F-statistic: 49.09 on 1 and 206 DF, p-value: $3.413 e-11$

round (confint (pers1), 3)

$\begin{array}{llrr}\text { \#\# } & & 2.5 \% & 97.5 \% \\ \text { \#\# (Intercept) } & 0.079 & 0.238 \\ \text { \#\# perseveration.s c } & 0.326 & 0.582\end{array}$

\# Moderation model

pers2 = lm(perseveration.i_c $\sim$ perseveration.s_c*gen.z, data $=$ pp.full)

summary (pers2)

\#\#

\#\# Call:

\#\# lm (formula = perseveration.i_c $\sim$ perseveration.s_c ${ }^{*}$ gen.z, data $=$ pp.full)

\#\#

\#\# Residuals:

\#\# Min $\quad$ 1Q Median $\quad 3 Q \quad$ Max

$\begin{array}{llllll}\# \# & -1.32291 & -0.41588 & -0.02321 & 0.38884 & 1.48517\end{array}$

\#\#

\#\# Coefficients:

\#\# Estimate Std. Error t value $\operatorname{Pr}(>|t|)$

\#\# (Intercept) $\quad 0.16357 \quad 0.04194 \quad 3.900 \quad 0.000131 * * *$

\#\# perseveration.s_c $\quad 0.32875 \quad 0.06877 \quad 4.780 \quad 3.35 e-06 * * *$

\#\# gen.z $0.19238 \quad 0.04355 \quad 4.4181 .62 e-05 * * *$

\#\# perseveration.s_c:gen.z $-0.02000 \quad 0.06053 \quad-0.330 \quad 0.741413$

\#\# ---

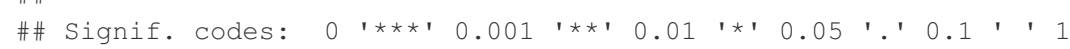

\#\#

\#\# Residual standard error: 0.5602 on 204 degrees of freedom

\#\# Multiple R-squared: 0.2636, Adjusted R-squared: 0.2528

\#\# F-statistic: 24.34 on 3 and 204 DF, p-value: 1.665e-13

round (confint (pers2), 3)

\begin{tabular}{|rrr}
$\# \#$ & $2.5 \div$ & $97.5 \%$ \\
$\# \#$ (Intercept) & 0.081 & 0.246 \\
$\# \#$ perseveration.s_c & 0.193 & 0.464 \\
\#\# gen.z & 0.107 & 0.278 \\
\#\# perseveration.s_c:gen.z & -0.139 & 0.099 \\
\hline
\end{tabular}

anova (pers1, pers2) 


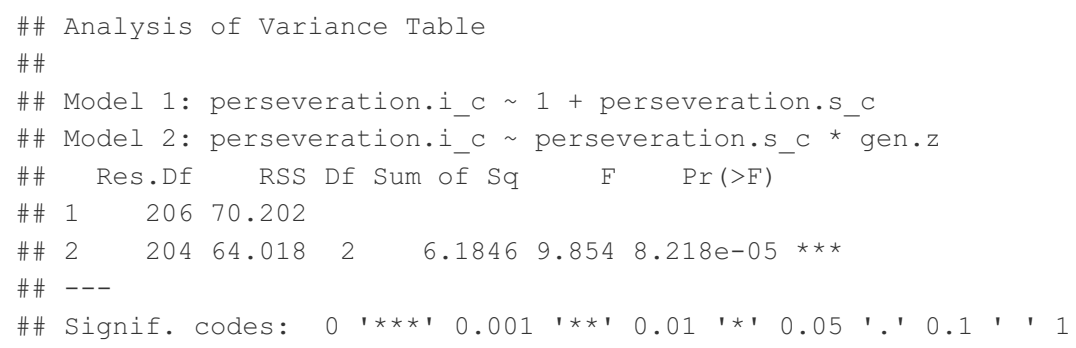

wilcox.test (pp.full\$restrictedaffectivity.s, pp.full\$restrictedaffectivity.i, paired = T)

\section{\#\#}

\#\# Wilcoxon signed rank test with continuity correction

\#\#

\#\# data: pp.full\$restrictedaffectivity.s and pp.full\$restrictedaffectivity.i

\#\# $V=5334.5$, p-value $=2.204 e-08$

\#\# alternative hypothesis: true location shift is not equal to 0

$(6.0661 / \operatorname{sqrt}(207))$

\#\# [1] 0.4216231

pnorm $(.422)$

\#\# [1] 0.6634875

$(2 * \operatorname{pnorm}((-\operatorname{abs}(.422)) / 2))$

\# [ [1] 0.8328873

print(corr.test(pp.full\$restrictedaffectivity.s, pp.full\$restrictedaffectivity.i, method = "spearman"), shor $t=\mathrm{F}$, digits $=3$ )

\#\# Call:corr.test $(\mathrm{x}=\mathrm{pp}$.full\$restrictedaffectivity.s, $\mathrm{y}=$ pp.full\$restrictedaffectivity.i,

\#\# method = "spearman")

\#\# Correlation matrix

\#\# [1] 0.203

\#\# Sample Size

\#\# [1] 207

\#\# Probability values adjusted for multiple tests.

\#\# [1] 0.003

\#\#

\#\# Confidence intervals based upon normal theory. To get bootstrapped values, try cor.ci

\#\# raw.lower raw.r raw.upper raw.p lower.adj upper.adj
\#\# NA-NA
0.0690 .203
0.330 .003
0.069
0.33 
\# Base model

pp.full\$restrictedaffectivity.s_c = pp.full\$restrictedaffectivity.s - mean(pp.full\$restrictedaffectivity.s) pp.full\$restrictedaffectivity.i_c = pp.full\$restrictedaffectivity.i - mean(pp.full\$restrictedaffectivity.s)

res1 = lm(restrictedaffectivity.i_c 1 + restrictedaffectivity.s_c, data = pp.full)

summary (res 1$)$

\#\#

\# \# Call:

\#\# Im(formula = restrictedaffectivity.i_c 1 + restrictedaffectivity.s_c,

\#\# data $=$ pp.full)

\#\#

\#\# Residuals:

\#\# Min 10 Median $30 \quad$ Max

$\begin{array}{llllll}\#-1.18496 & -0.44854 & -0.02106 & 0.38702 & 1.98004\end{array}$

\#\#

\#\# Coefficients:

\#\# Estimate std. Error t value $\operatorname{Pr}(>|t|)$

\#\# (Intercept) $0.32565 \quad 0.04507 \quad 7.2269 .62 e-12$ ***

\#\# restrictedaffectivity.s_c $0.28681 \quad 0.07849 \quad 3.654 \quad 0.000328$ ***

\#\# ---

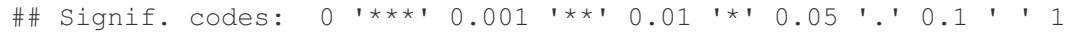

\#\#

\#\# Residual standard error: 0.6484 on 205 degrees of freedom

\#\# (1 observation deleted due to missingness)

\#\# Multiple R-squared: 0.06115 , Adjusted R-squared: 0.05657

\#\# F-statistic: 13.35 on 1 and 205 DF, p-value: 0.0003279

round (confint (res1), 3)

$\begin{array}{lrr}\text { \#\# } & 2.5 \div 97.5 \% \\ \# \# \text { (Intercept) } & 0.237 & 0.414 \\ \text { \#\# restrictedaffectivity.s_c } & 0.132 & 0.442\end{array}$

\# Moderation model

res2 = lm(restrictedaffectivity.i_c $\sim$ restrictedaffectivity.s_c*gen.z, data = pp.full)

summary (res2)

\#\#

\#\# Call:

\#\# $\operatorname{lm}($ formula = restrictedaffectivity.i_c $\sim$ restrictedaffectivity.s_c *

\#\# gen.z, data $=$ pp.full)

\#\#

\#\# Residuals:

\#\# Min $1 Q \quad$ Median $3 Q \quad$ Max

$\begin{array}{llllll}\# \# & -1.22323 & -0.42525 & -0.04537 & 0.38040 & 1.94791\end{array}$

\#\#

\#\# Coefficients:

\#\#

\#\# (Intercept)

\#\# restrictedaffectivity.s_

Estimate Std. Error $t$ value $\operatorname{Pr}(>|t|)$

$0.34158 \quad 0.04626 \quad 7.384 \quad 3.89 e-12 * * *$

\#\# restrictedaffectivity.s_c:gen.z $-0.09613 \quad 0.07916 \quad-1.214 \quad 0.22601$

\#\# ---

\#\# Signif. codes: 0 '***' 0.001 '**' 0.01 '*' 0.05 '.' 0.1 ' ' 1

\#\#

\#\# Residual standard error: 0.6354 on 203 degrees of freedom

\#\# (1 observation deleted due to missingness)

\#\# Multiple R-squared: 0.107, Adjusted R-squared: 0.09385

\#\# F-statistic: 8.111 on 3 and 203 DF, p-value: 3.964 e-05

round (confint (res2), 3) 


$\begin{array}{lrr}\# \# & 2.5 \% & 97.5 \% \\ \# \# \text { (Intercept) } & 0.250 & 0.433 \\ \text { \#\# restrictedaffectivity.s_c } & 0.071 & 0.397 \\ \text { \#\# gen.z } & 0.052 & 0.237 \\ \text { \#\# restrictedaffectivity.s_c:gen.z } & -0.252 & 0.060\end{array}$

anova (res1, res2)

\#\# Analysis of Variance Table

\#\#

\#\# Model 1: restrictedaffectivity.i_c $~ 1$ + restrictedaffectivity.s_c

\#\# Model 2: restrictedaffectivity.i c restrictedaffectivity.s c * gen.z

\#\# Res.Df RSS Df Sum of Sq F $\operatorname{Pr}(>\mathrm{F})$

\#\# $1205 \quad 86.178$

\#\# $2 \quad 203 \quad 81.965 \quad 2 \quad 4.2124 \quad 5.2164 \quad 0.006178$ **

\#\# ---

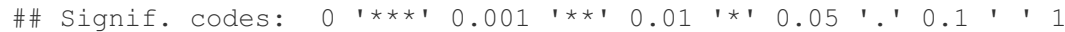

\#\# Rigid perfectionism \#\#

t.test(pp.full\$rigidperfectionism.s, pp.full\$rigidperfectionism.i, paired = T)

\#\#

\# Paired t-test

\#\#

\#\# data: pp.full\$rigidperfectionism.s and pp.full\$rigidperfectionism.i

$\# \#=-1.1566, \mathrm{df}=207, \mathrm{p}$-value $=0.2488$

\#\# alternative hypothesis: true difference in means is not equal to 0

\#\# 95 percent confidence interval:

$\begin{array}{lll}\text { \#\# }-0.17127833 & 0.04462235\end{array}$

\#\# sample estimates:

\#\# mean of the differences

\#\#

$-0.06332799$

wilcox.test (pp.full\$rigidperfectionism.s, pp.full\$rigidperfectionism.i, paired = T)

\#\#

\#\# Wilcoxon signed rank test with continuity correction

\#\#

\#\# data: pp.full\$rigidperfectionism.s and pp.full\$rigidperfectionism.i

\#\# $\mathrm{V}=9063, \mathrm{p}$-value $=0.2286$

\#\# alternative hypothesis: true location shift is not equal to 0

$(1.1566 /$ sqrt (208))

\#\# [1] 0.08019578

pnorm $(.080)$

\#\# [1] 0.5318814

$(2 * \operatorname{pnorm}((-\operatorname{abs}(.080)) / 2))$

\#\# [1] 0.9680931

print(corr.test(pp.full\$rigidperfectionism.s, pp.full\$rigidperfectionism.i, method = "spearman"), short = F, digits $=3$ ) 
\#\# Call:corr.test( $\mathrm{x}=$ pp.full\$rigidperfectionism.s, $y=$ pp.full\$rigidperfectionism.i,

\#\# method = "spearman")

\#\# Correlation matrix

\#\# [1] 0.296

\#\# Sample Size

\#\# [1] 208

\#\# Probability values adjusted for multiple tests.

\#\# [1] 0

\#\#

\#\# Confidence intervals based upon normal theory. To get bootstrapped values, try cor.ci

\#\# raw.lower raw.r raw.upper raw.p lower.adj upper.adj
\#\# NA-NA
0.1670 .296
0.415
0
0.167
0.415

\section{\# Base model}

pp.full\$rigidperfectionism.s_c = pp.full\$rigidperfectionism.s - mean(pp.full\$rigidperfectionism.s) pp.full\$rigidperfectionism.i_c = pp.full\$rigidperfectionism.i - mean(pp.full\$rigidperfectionism.s)

rig1 = lm(rigidperfectionism.i_c 1 + rigidperfectionism.s_c, data $=$ pp.full)

summary (rig1)

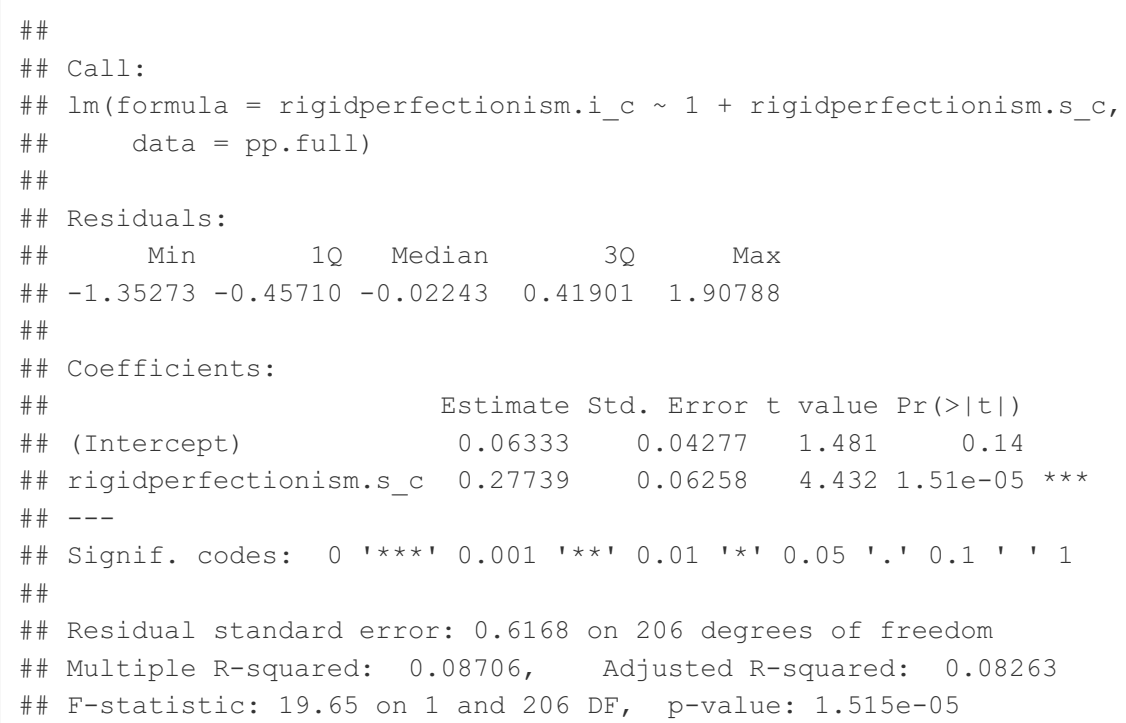

round (confint (rig1), 3)

\begin{tabular}{|rrr}
$\# \#$ & $2.5 \%$ & $97.5 \%$ \\
$\# \#$ (Intercept) & -0.021 & 0.148 \\
$\# \#$ rigidperfectionism.s_c & 0.154 & 0.401 \\
\hline
\end{tabular}

\# Moderation model

rig2 = lm(rigidperfectionism.i_c $~$ rigidperfectionism.s_c*gen.z, data = pp.full) summary (rig2) 
\#\# Call:

\#\# $\operatorname{lm}$ (formula = rigidperfectionism.i_c $\sim$ rigidperfectionism.s_c *

\#\# gen.z, data $=$ pp.full)

\#\#

\#\# Residuals:

\#\# Min $\quad 1 Q \quad$ Median $\quad 30 \quad$ Max

$\begin{array}{llllll}\# \# & -1.42854 & -0.41037 & -0.02533 & 0.42146 & 2.05267\end{array}$

\#\#

\#\# Coefficients:

\#\#

\#\# (Intercept)

\#\# rigidperfectionism.s_c

\#\# gen.z

$\begin{array}{llll}0.05934 & 0.04277 & 1.387 & 0.16685\end{array}$

$\begin{array}{llll}0.18053 & 0.06390 & 2.825 & 0.00519 * *\end{array}$

$\begin{array}{llllll}\text { \# } \text { rigidperfectionism.s_c:gen.z } & 0.01804 & 0.05628 & 0.321 & 0.74887\end{array}$

\#\# ---

\#\# Signif. codes: 0 '***' 0.001 '**' 0.01 '*' 0.05 '.' 0.1 ' ' 1

\#\#

\#\# Residual standard error: 0.5901 on 204 degrees of freedom

\#\# Multiple R-squared: 0.1725, Adjusted R-squared: 0.1603

\#\# F-statistic: 14.18 on 3 and 204 DF, p-value: $1.989 e-08$

round (confint (rig2), 3)

\begin{tabular}{|c|c|c|c|}
\hline \#\# & & $2.5 \div$ & $97.5 \%$ \\
\hline \#\# & (Intercept) & -0.025 & 0.144 \\
\hline \#\# & rigidperfectionism.s_c & 0.055 & 0.307 \\
\hline \#\# & gen.z & 0.111 & 0.283 \\
\hline \#\# & rigidperfectionism.s_c:gen.z & -0.093 & 0.12 \\
\hline
\end{tabular}

anova (rig1, rig2)

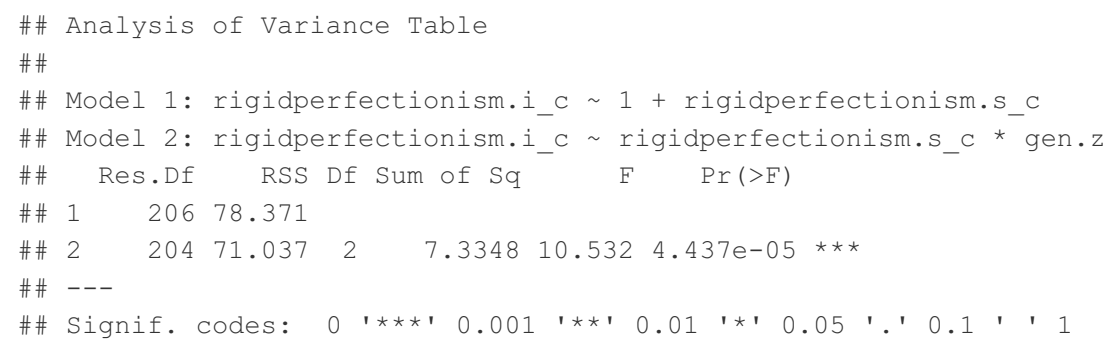

wilcox.test (pp.full\$risktaking.s, pp.full\$risktaking.i, paired $=\mathrm{T}$ ) 
\#\# [1] 0.08638793

pnorm $(.086)$

\#\# [1] 0.5342668

$(2 * \operatorname{pnorm}((-\mathrm{abs}(.086)) / 2))$

\# \# [1] 0.9657015

print(corr.test (pp.full\$risktaking.s, pp.full\$risktaking.i, method = "spearman"), short = F, digits = 3)

\#\# Call:corr.test $(x=$ pp.full\$risktaking.s, $y=p p . f u l l \$ r i s k t a k i n g . i$,

\#\# method = "spearman")

\#\# Correlation matrix

\#\# [1] 0.38

\#\# Sample Size

\#\# [1] 206

\#\# Probability values adjusted for multiple tests.

\#\# [1] 0

\#\#

\#\# Confidence intervals based upon normal theory. To get bootstrapped values, try cor.ci

\#\# raw.lower raw.r raw.upper raw.p lower.adj upper.adj

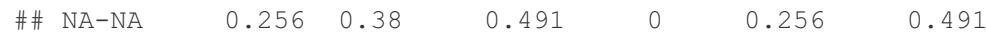

\# Base model

pp.full\$risktaking.s_c = pp.full\$risktaking.s - mean (pp.full\$risktaking.s)

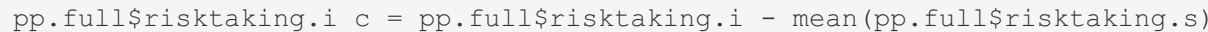

ril $=\operatorname{lm}($ risktaking.i_c $\sim 1+$ risktaking.s_c, data $=$ pp.full)

summary (ri1)

\#\#

\#\# Call:

\#\# $\operatorname{lm}($ formula $=$ risktaking.i_c $\sim 1$ +risktaking.s_c, data $=$ pp.full)

\#\#

\#\# Residuals:

\#\# $\quad$ Min Median $30 \quad$ Max

$\begin{array}{llllll}\# \# & -1.2315 & -0.3450 & -0.0151 & 0.3014 & 1.4870\end{array}$

\#\#

\#\# Coefficients:

\#\# Estimate Std. Error $t$ value $\operatorname{Pr}(>|t|)$

$\begin{array}{lllll}\text { \# \# (Intercept) } & 0.04882 & 0.03617 & 1.350 & 0.179\end{array}$

\#\# risktaking.s_c $0.50991 \quad 0.07397 \quad 6.894 \quad 6.66 \mathrm{e}-11$ ***

\#\# ---

\#\# Signif. codes: 0 '***' 0.001 '**' 0.01 '*' 0.05 '.'0.1' ' 1

\#\#

\#\# Residual standard error: 0.5191 on 204 degrees of freedom

\#\# (2 observations deleted due to missingness)

\#\# Multiple R-squared: 0.1889, Adjusted R-squared: 0.185

\#\# F-statistic: 47.52 on 1 and 204 DF, p-value: 6.657e-11

round (confint (ri1), 3)

$\begin{array}{llrr}\text { \#\# } & & 2.5 \% & 97.5 \% \\ \text { \#\# } \quad \text { (Intercept) } & -0.022 & 0.120 \\ \text { \#\# risktaking.s C } & 0.364 & 0.656\end{array}$

\# Moderation model

ri2 = lm(risktaking.i $c \sim$ risktaking.s $c^{\star} g e n \cdot z$, data $=$ pp.full)

summary (ri2) 
\#\# [1] 0.2633092

$\operatorname{pnorm}(.263)$

\#\# [1] 0.6037247

$(2 * \operatorname{pnorm}((-\operatorname{abs}(.263)) / 2))$

\#\# [1] 0.8953798

print(corr.test(pp.full\$separationinsecurity.s, pp.full\$separationinsecurity.i, method = "spearman"), short

$=\mathrm{F}, \operatorname{digits}=3$ )

\#\# Call:corr.test $(\mathrm{x}=$ pp.full\$separationinsecurity.s, $y=$ pp.full\$separationinsecurity.i,

\#\# method = "spearman")

\#\# Correlation matrix

\#\# [1] 0.404

\#\# Sample Size

\#\# [1] 208

\#\# Probability values adjusted for multiple tests.

\#\# [1] 0

\#\#

\#\# Confidence intervals based upon normal theory. To get bootstrapped values, try cor.ci

\#\# raw.lower raw.r raw.upper raw.p lower.adj upper.adj
\#\# NA-NA
0.2830 .404
0.512
0.283
0.512

\# Base model

pp.full\$separationinsecurity.s c = pp.full\$separationinsecurity.s - mean (pp.full\$separationinsecurity.s)

pp.full\$separationinsecurity.i_c = pp.full\$separationinsecurity.i - mean (pp.full\$separationinsecurity.s)

sep1 = lm(separationinsecurity.i c 1 + separationinsecurity.s c, data = pp.full)

summary (sep1)

\#\#

\#\# Call:

\#\# Im(formula = separationinsecurity.i c 1 + separationinsecurity.s c,

\#\# data $=$ pp.full

\#\#

\#\# Residuals:

\#\# Min 10 Median 30 Max

$\begin{array}{llllll}\# \# & -1.4009 & -0.5111 & -0.1227 & 0.3773 & 1.6934\end{array}$

\#\#

\#\# Coefficients:

\#\# Estimate Std. Error t value Pr $(>|t|)$

\#\# (Intercept) $0.19929 \quad 0.04589 \quad 4.3422 .21 e^{-05}$ ***

\#\# separationinsecurity.s_c $0.42518 \quad 0.07148 \quad 5.9481 .15 e-08$ ***

\#\# ---

\#\# Signif. codes: 0 '***' 0.001 '**' 0.01 '*' 0.05 '.'0.1 ' ' 1

\#\#

\#\# Residual standard error: 0.6619 on 206 degrees of freedom

\#\# Multiple R-squared: 0.1466, Adjusted R-squared: 0.1424

\#\# F-statistic: 35.38 on 1 and 206 DF, p-value: $1.147 e-08$

round (confint (sep1), 3)

$\begin{array}{lrr}\text { \#\# } & 2.5 \div 97.5 \% \\ \# \# \text { (Intercept) } & 0.109 & 0.290 \\ \text { \#\# separationinsecurity.s_c } & 0.284 & 0.566\end{array}$


\#\# Wilcoxon signed rank test with continuity correction

\#\# data: pp.full\$submissiveness.s and pp.full\$submissiveness.i

\#\# $\mathrm{V}=5588, \mathrm{p}$-value $=0.006237$

\#\# alternative hypothesis: true location shift is not equal to 0

\section{(2.9094/sqrt (197))}

\#\# [1] 0.2072862

pnorm (.207)

\# \# [1] 0.5819951

$(2 * \operatorname{pnorm}((-\operatorname{abs}(.207)) / 2))$

\# [1] 0.9175661

print(corr.test (pp.full\$submissiveness.s, pp.full\$submissiveness.i, method = "spearman"), short = F, digits $=3)$

\#\# Call:corr.test $(x=$ pp.full\$submissiveness.s, $y=$ pp.full\$submissiveness.i,

\#\# method = "spearman")

\#\# Correlation matrix

\#\# [1] 0.207

\#\# Sample Size

\#\# [1] 197

\#\# Probability values adjusted for multiple tests.

\#\# [1] 0.003

\#\#

\# Confidence intervals based upon normal theory. To get bootstrapped values, try cor.ci

\#\# raw.lower raw.r raw.upper raw.p lower.adj upper.adj
\#\# NA-NA
0.070 .207
0.3370 .003
0.07
0.337

\# Base model

pp.full\$submissiveness.s_c $=$ pp.full\$submissiveness.s - mean (pp.full\$submissiveness.s, na.rm = T)

pp.full\$submissiveness.i_c $=$ pp.full\$submissiveness.i - mean (pp.full\$submissiveness.s, na.rm = T)

sub1 = lm(submissiveness.i_c $1+$ submissiveness.s_c, data $=$ pp.full)

summary (sub1)

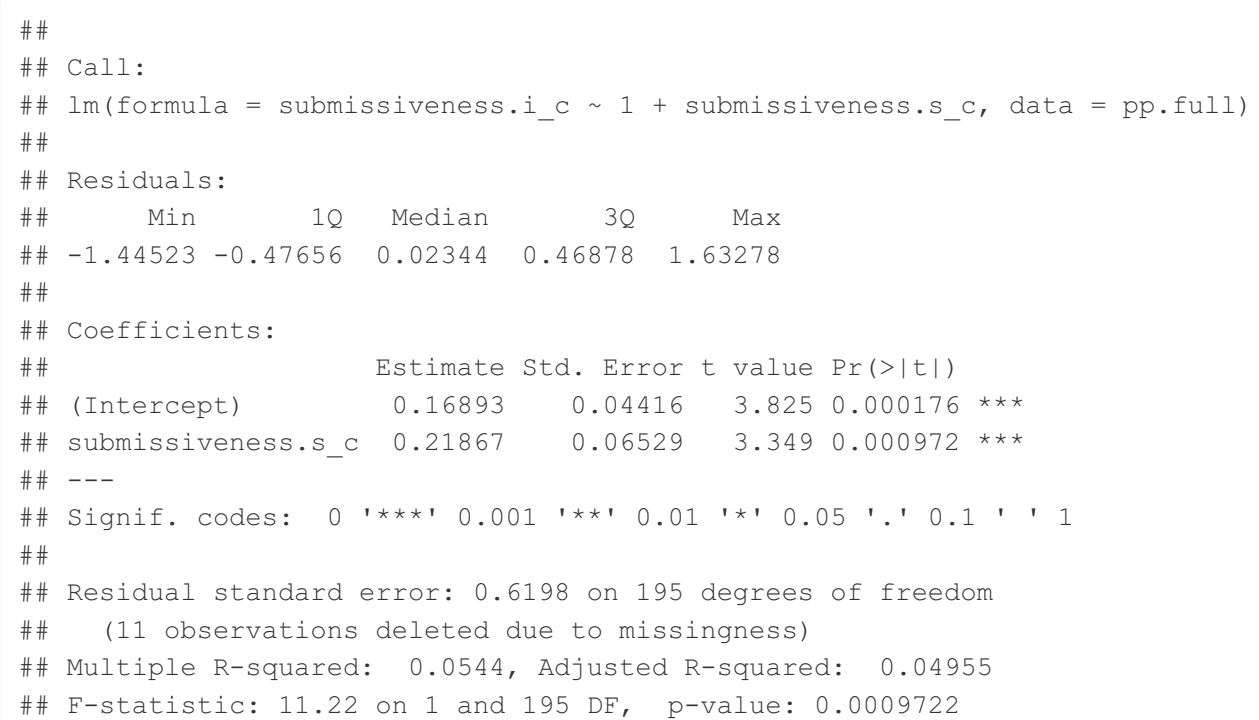




$\begin{array}{lrr}\text { \#\# } & 2.5 \% & 97.5 \% \\ \text { \#\# (Intercept) } & 0.082 & 0.256 \\ \text { \#\# submissiveness.s_c } & 0.090 & 0.347\end{array}$

\# Moderation model

sub2 = lm(submissiveness.i_c $\sim$ submissiveness.s_c*(gen.z), data $=$ pp.full)

summary (sub2)

\section{\#\#}

\#\# Call:

\#\# Im(formula = submissiveness.i_c submissiveness.s_c * (gen.z),

\#\# data $=$ pp.full)

\#\#

\#\# Residuals:

\#\# Min $\quad 1 Q \quad$ Median $\quad 30 \quad$ Max

$\begin{array}{llllll}\# \# & -1.62408 & -0.45014 & 0.00816 & 0.44976 & 1.63534\end{array}$

\#\#

\#\# Coefficients:

\#\# Estimate std. Error t value Pr $(>|t|)$

\#\# (Intercept) $0.17041 \quad 0.04365 \quad 3.9040 .000131$ ***

\#\# submissiveness.s_c $\quad 0.20378 \quad 0.06480 \quad 3.1450 .001926$ **

$\begin{array}{llllll}\text { \# } \text { gen.z } & 0.03843 & 0.04419 & 0.870 & 0.385582\end{array}$

\#\# submissiveness.s_c:gen.z $-0.15187 \quad 0.06369-2.3850 .018061$ *

\#\# ---

\#\# Signif. codes: 0 '***' 0.001 '**' 0.01 '*' 0.05 '.' 0.1 ' ' 1

\#\#

\#\# Residual standard error: 0.6126 on 193 degrees of freedom

\#\# (11 observations deleted due to missingness)

\#\# Multiple R-squared: 0.08576, Adjusted R-squared: 0.07154

\#\# F-statistic: 6.034 on 3 and 193 DE, p-value: 0.0005992

round (confint (sub2), 3)

$\begin{array}{rrr}\# \# & 2.5 \% & 97.5 \% \\ \# \# \text { (Intercept) } & 0.084 & 0.257 \\ \text { \#\# submissiveness.s_c } & 0.076 & 0.332 \\ \text { \#\# gen.z } & -0.049 & 0.126 \\ \text { \#\# submissiveness.s_c:gen.z } & -0.277 & -0.026\end{array}$

\# Test of simple slopes

sim_slopes(sub2, pred $=$ submissiveness.s_c, modx $=$ gen.z, modx.values $=\mathrm{c}(-2.5,-2,-1.5,-1,-.5,0, .5,1$

$, 1.5,2,2.5,3)$,

control.fdr $=\mathrm{T}$ )

\#\# JOHNSON-NEYMAN INTERVAL

\#\#

\#\# When gen.z is OUTSIDE the interval [0.32, 23.54], the slope of

\#\# submissiveness.s_c is $p<.05$.

\#\#

\#\# Note: The range of observed values of gen.z is $[-2.69,3.01]$

\#\#

\#\# Interval calculated using false discovery rate adjusted $t=2.24$

\#\#

\#\# SIMPLE SLOPES ANALYSiS

\#\#

\#\# Slope of submissiveness.s c when gen.z $=3.00$ :

\#\#

\#\# Est. S.E. $t$ val. p

\#\#----- $-----\quad------\quad-----$

$\begin{array}{lllll}\# \# & -0.25 & 0.21 & -1.21 & 0.23\end{array}$

\#\#

\#\# Slope of submissiveness.s_c when gen.z $=2.50$ :

\#\#

\#\# Est. S.E. $t$ val. $p$

\#\# ----- $-----\quad------\quad----$

$\begin{array}{lllll}\# \# & -0.18 & 0.18 & -0.99 & 0.32\end{array}$

\#\# 
Slope of submissiveness.s_c when gen.z $=2.00$ :

$\# \#$

\#\# Est. S.E. $t$ val. p

$\begin{array}{rrrrr}\# \# & ------ & ----- & ------- & ----- \\ \# \# & -0.10 & 0.15 & -0.68 & 0.50\end{array}$

$\# \#$

\#\# Slope of submissiveness.s_c when gen.z $=1.50$ :

\#\#

\#\# Est. S.E. $t$ val. $p$

$\begin{array}{rrrrr}\# \# & ------ & ----- & ------- & ----- \\ \# \# & -0.02 & 0.12 & -0.20 & 0.84\end{array}$

\#\#

\#\# Slope of submissiveness.s_c when gen.z $=1.00$ :

\#\#

\#\# Est. S.E. $t$ val. p

$\begin{array}{rrrrr}\text { \#\# } & ----- & ----- & ------ & ---- \\ \# \# & 0.05 & 0.09 & 0.55 & 0.59\end{array}$

\#\#

\#\# Slope of submissiveness.s_c when gen.z $=0.50$ :

\#\#

\#\# Est. S.E. $t$ val. p

\#\# ----- $-----1-------\quad-----$

$\begin{array}{lllll}\# \# & 0.13 & 0.07 & 1.71 & 0.09\end{array}$

\#\#

\#\# Slope of submissiveness.s $c$ when gen.z $=0.00$ :

\#\#

\#\# Est. S.E. t val. p

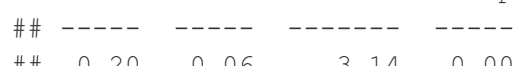

$\begin{array}{lllll}\# \quad 0.20 & 0.06 & 3.14 & 0.00\end{array}$

\#\#

\#\# Slope of submissiveness.s_c when gen.z $=-0.50$ :

\#\#

\#\# Est. S.E. $t$ val. p

\#\# ----- $-----\quad-------\quad-----$

$\begin{array}{lllll}\# \# & 0.28 & 0.07 & 4.02 & 0.00\end{array}$

\#\#

\#\# Slope of submissiveness.s_c when gen.z $=-1.00$ :

\#\#

\#\# Est. S.E. $t$ val. $p$

$\begin{array}{ccccc}\# \#----- & ----- & ------- & ----- \\ \# \# & 0.36 & 0.09 & 4.11 & 0.00\end{array}$

\#\# $0.36 \quad 0.09$

\#\#

\#\# Slope of submissiveness.s_c when gen.z $=-1.50$ :

\#\#

\#\# Est. S.E. $t$ val. p

\#\# ----- -----

$\begin{array}{lllll}\# \# & 0.43 & 0.11 & 3.91 & 0.00\end{array}$

\#\#

\#\# Slope of submissiveness.s_c when gen.z $=-2.00$ :

\#\#

\#\# Est. S.E. $t$ val. $p$

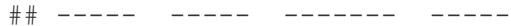

$\begin{array}{lllll}\# \# & 0.51 & 0.14 & 3.69 & 0.00\end{array}$

\#\#

\#\# Slope of submissiveness.s_c when gen.z $=-2.50$ :

\#\#

\#\# Est. S.E. $t$ val. p

$\begin{array}{rrrrr}\text { \#\# } & ----- & ----- & ------- & ---- \\ \text { \#\# } & 0.58 & 0.17 & 3.51 & 0.00\end{array}$ 


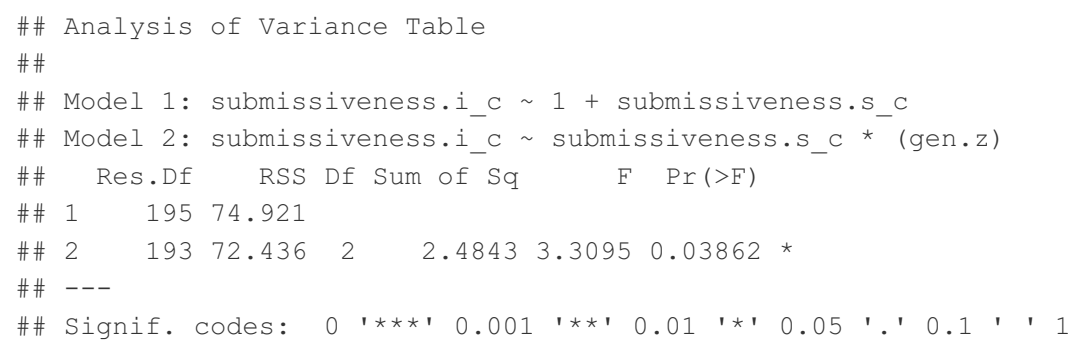

\section{\#\# Suspiciousness \#\#}

t.test(pp.full\$suspiciousness.s, pp.full\$suspiciousness.i.ACCURATE, paired = T)

\section{\#\#}

\#\# Paired t-test

\#\#

\#\# data: pp.full\$suspiciousness.s and pp.full\$suspiciousness.i.ACCURATE

\#\# $t=-0.49401, \mathrm{df}=207, \mathrm{p}$-value $=0.6218$

\#\# alternative hypothesis: true difference in means is not equal to 0

\#\# 95 percent confidence interval:

\#\# $\quad-0.11905591 \quad 0.07134529$

\#\# sample estimates:

\#\# mean of the differences

\#\# $\quad-0.02385531$

wilcox.test (pp.full\$suspiciousness.s, pp.full\$suspiciousness.i.ACCURATE, paired = T)

\#\#

\#\# Wilcoxon signed rank test with continuity correction

\#\#

\#\# data: pp.full\$suspiciousness.s and pp.full\$suspiciousness.i.ACCURATE

\#\# $\mathrm{V}=10092$, p-value $=0.944$

\#\# alternative hypothesis: true location shift is not equal to 0

$(.49401 /$ sqrt (208))

\#\# [1] 0.03425343

pnorm (.034)

\#\# [1] 0.5135614

$(2 * \operatorname{pnorm}((-\operatorname{abs}(.034)) / 2))$

\# \# [1] 0.9864366

print(corr.test(pp.full\$suspiciousness.s, pp.full\$suspiciousness.i.ACCURATE, method = "spearman"), short = F , digits $=3)$

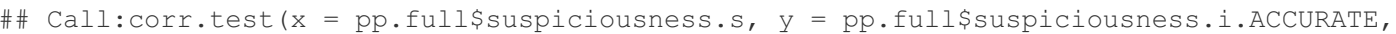

\#\# method = "spearman")

\#\# Correlation matrix

\#\# [1] 0.387

\#\# Sample Size

\#\# [1] 208

\#\# Probability values adjusted for multiple tests.

\#\# [1] 0

\#\#

\#\# Confidence intervals based upon normal theory. To get bootstrapped values, try cor.ci

\#\# raw.lower raw.r raw.upper raw.p lower.adj upper.adj

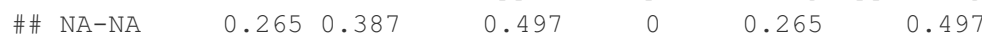


* Base model

pp.full\$suspiciousness.s_c = pp.full\$suspiciousness.s - mean(pp.full\$suspiciousness.s)

pp.full\$suspiciousness.i_c $=$ pp.full\$suspiciousness.i - mean (pp.full\$suspiciousness.s)

suspil = lm(suspiciousness.i_c $1+$ suspiciousness.s_c, data $=$ pp.full)

summary (suspi1)

\#\#

\# \# Call:

\#\# lm(formula = suspiciousness.i_c $1+$ suspiciousness.s_c, data $=$ pp.full)

\#\#

\#\# Residuals:

\#\# Min 12 Median $3 Q \quad$ Max

$\begin{array}{llllll}\# \# & -1.1260 & -0.3890 & -0.0091 & 0.3894 & 1.9048\end{array}$

\#\#

\#\# Coefficients:

\#\# Estimate Std. Error $t$ value $\operatorname{Pr}(>|t|)$

\#\# (Intercept) $0.10440 \quad 0.03926 \quad 2.659 \quad 0.00845 * *$

\#\# suspiciousness.s_c $0.40196 \quad 0.06403 \quad 6.278 \quad 2 e-09 * * *$

\#\#---

\#\# Signif. codes: 0 '***' 0.001 '**' 0.01 '*' 0.05 '.' 0.1 ' ' 1

\#\#

\#\# Residual standard error: 0.5662 on 206 degrees of freedom

\#\# Multiple R-squared: 0.1606, Adjusted R-squared: 0.1565

\#\# F-statistic: 39.41 on 1 and 206 DF, p-value: $1.997 e-09$

round (confint (suspi1), 3)

$\begin{array}{llrr}\# \# & 2.5 \div 97.5 \% \\ \# \# \text { (Intercept) } & 0.027 & 0.182 \\ \text { \#\# } & \text { suspiciousness.s c } & 0.276 & 0.528\end{array}$

\# Moderation model

suspi2 = lm(suspiciousness.i_c $\sim$ suspiciousness.s_c*gen.z, data $=$ pp.full)

summary (suspi2)

\#\#

\#\# Call:

\#\# Im(formula = suspiciousness.i_c $\sim$ suspiciousness.s_c * gen.z,

\#\# data $=$ pp.full)

\#\#

\#\# Residuals:

\#\# $\quad$ Min $\quad 10$ Median $\quad 30 \quad$ Max

$\begin{array}{llllll}\# \# & -1.49340 & -0.33942 & -0.02325 & 0.37874 & 1.35419\end{array}$

\#\#

\#\# Coefficients:

\#\#

\#\# (Intercept)

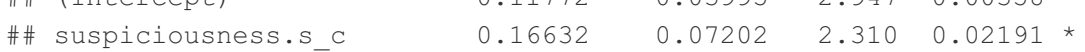

\#\# gen.z $0.27866 \quad 0.04335 \quad 6.4298 .95 e-10 * * *$

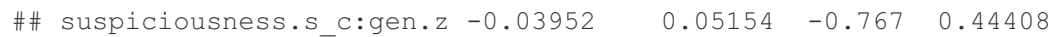

$\#$ \# ---

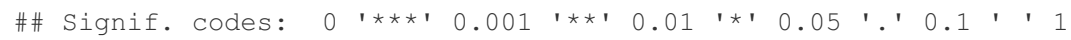

\#\#

\#\# Residual standard error: 0.5188 on 204 degrees of freedom

\#\# Multiple R-squared: 0.3022, Adjusted R-squared: 0.2919

\#\# F-statistic: 29.45 on 3 and 204 DF, p-value: $7.311 e-16$

round (confint (suspi2), 3)

$\begin{array}{rlrr}\text { \#\# } & 2.5 \% & 97.5 \% \\ \# \# \text { (Intercept) } & 0.039 & 0.196 \\ \text { \#\# suspiciousness.s_c } & 0.024 & 0.308 \\ \text { \#\# gen.z } & 0.193 & 0.364 \\ \text { \#\# suspiciousness.s_c:gen.z } & -0.141 & 0.062\end{array}$


\#\# Analysis of Variance Table

\#\#

\#\# Model 1: suspiciousness.i_c 1 + suspiciousness.s_c

\#\# Model 2: suspiciousness.i_c $\sim$ suspiciousness.s_c * gen.z

\#\# Res.Df RSS Df Sum of Sq F $\operatorname{Pr}(>\mathrm{F})$

\#\# $1206 \quad 66.042$

\#\# $2 \quad 20454.9012 \quad 11.14120 .698 \quad 6.541 e-09 * \star *$

\#\#---

\#\# Signif. codes: 0 '***' 0.001 '**' 0.01 '*' 0.05 '.' 0.1 ' ' 1

\#\# Unusual beliefs \#\#

t.test (pp.full\$unusualbelexp.s, pp.full\$unusualbelexp.i, paired $=T$ )

\#\#

\#\# Paired t-test

\#\#

\#\# data: pp.full\$unusualbelexp.s and pp.full\$unusualbelexp.i

\#\# $\mathrm{t}=1.6135, \mathrm{df}=205, \mathrm{p}$-value $=0.1082$

\#\# alternative hypothesis: true difference in means is not equal to 0

\#\# 95 percent confidence interval:

\#\# -0.018894820 .18914448$

\#\# sample estimates:

\#\# mean of the differences

\#\#

0.08512483

wilcox.test (pp.full\$unusualbelexp.s, pp.full\$unusualbelexp.i, paired $=\mathrm{T}$ )

\#\#

\#\# Wilcoxon signed rank test with continuity correction

\#\#

\#\# data: pp.full\$unusualbelexp.s and pp.full\$unusualbelexp.i

\#\# $\mathrm{V}=7375, \mathrm{p}$-value $=0.1109$

\#\# alternative hypothesis: true location shift is not equal to 0

$(1.6135 /$ sqrt (206))

\#\# [1] 0.1124179

pnorm (.112)

\#\#[1] 0.5445883

$(2 * \operatorname{pnorm}((-\operatorname{abs}(.112)) / 2))$

\# \# [1] 0.9553418

print(corr.test(pp.full\$unusualbelexp.s, pp.full\$unusualbelexp.i, method = "spearman"), short = F, digits = 3) 


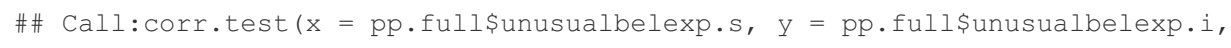

\#\# $\quad$ method = "spearman")

\#\# Correlation matrix

\#\# [1] 0.229

\#\# Sample Size

\#\# [1] 206

\#\# Probability values adjusted for multiple tests.

\#\# [1] 0.001

\#\#

\#\# Confidence intervals based upon normal theory. To get bootstrapped values, try cor.ci

\#\# raw.lower raw.r raw.upper raw.p lower.adj upper.adj
\#\# NA-NA
0.0960 .229
0.3550 .001
0.096
0.355

\section{\# Base model}

pp.full\$unusualbelexp.s_c = pp.full\$unusualbelexp.s - mean (pp.full\$unusualbelexp.s)

pp.full\$unusualbelexp.i_c $=$ pp.full\$unusualbelexp.i - mean(pp.full\$unusualbelexp.s)

unul $=\operatorname{lm}$ (unusualbelexp.i_c $\sim 1+$ unusualbelexp.s_c, data $=$ pp.full)

summary (unu1)

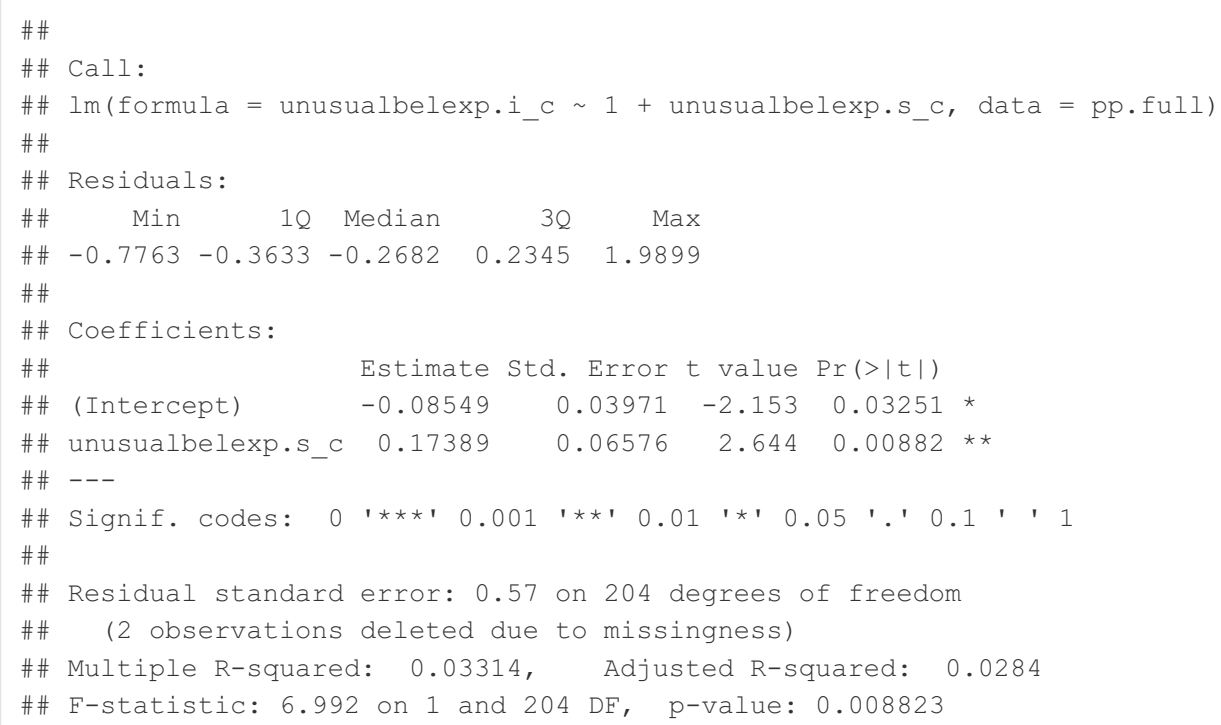

\begin{tabular}{|rrr}
\hline$\# \#$ & $2.5 \% 97.5 \%$ \\
$\# \#$ (Intercept) & -0.164 & -0.007 \\
\#\# unusualbelexp.s_c & 0.044 & 0.304
\end{tabular}

\# Moderation model

unu2 $=\operatorname{lm}$ (unusualbelexp.i_c $\sim$ unusualbelexp.s_c*gen.z, data $=$ pp.full) summary (unu2) 
\#\# Call:

\#\# $\operatorname{lm}($ formula $=$ unusualbelexp.i c $\sim$ unusualbelexp.s c $*$ gen.z, data $=$ pp.full)

\#\#

\#\# Residuals:

\#\# Min $1 Q$ Median $3 Q \quad$ Max

$\begin{array}{llllll}\# \# & -0.7043 & -0.3831 & -0.1919 & 0.2109 & 1.7486\end{array}$

\#\#

\#\# Coefficients:

\#\#

\#\# (Intercept)

Estimate std. Error $t$ value $\operatorname{Pr}(>|t|)$

\#\# gen.z $\quad 0.12621 \quad 0.04007 \quad 3.150 \quad 0.00188$ **

\#\# unusualbelexp.s_c:gen.z $-0.12885 \quad 0.07405-1.740 \quad 0.08337$.

$\# \#---$

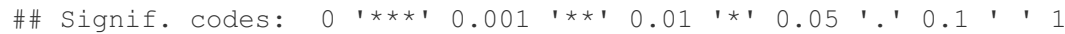

\#\#

\#\# Residual standard error: 0.5545 on 202 degrees of freedom

\#\# (2 observations deleted due to missingness)

\#\# Multiple R-squared: 0.09401, Adjusted R-squared: 0.08055

\#\# F-statistic: 6.987 on 3 and 202 DF, p-value: 0.0001705

round (confint (unu2), 3)

$\begin{array}{lrr}\# \# \text { \# } & 2.5 \div 97.5 \% \\ \# \text { (Intercept) } & -0.149 & 0.006 \\ \text { \#\# unusualbelexp.s_c } & 0.028 & 0.288 \\ \text { \#\# gen.z } & 0.047 & 0.205 \\ \text { \#\# unusualbelexp.s_c:gen.z } & -0.275 & 0.017\end{array}$

anova (unu1, unu2)

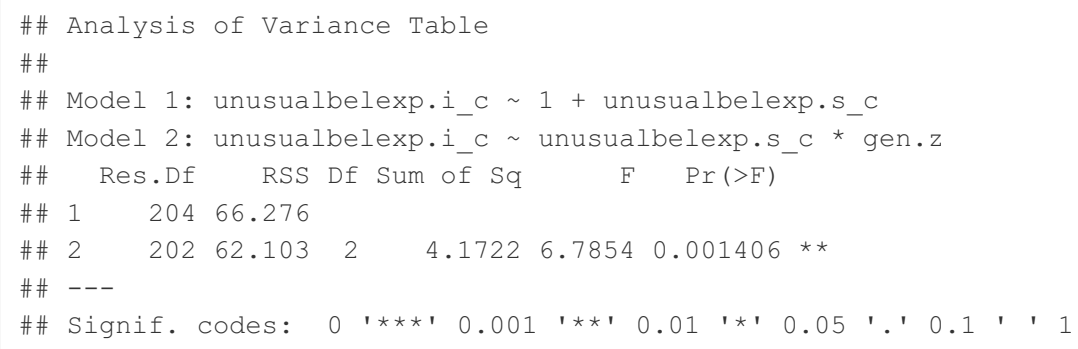

wilcox.test (pp.full\$withdrawal.s, pp.full\$withdrawal.i, paired $=\mathrm{T}$ ) 
\#\# [1] 0.03467015

pnorm (.035)

\#\# [1] 0.5139601

$(2 * \operatorname{pnorm}((-\operatorname{abs}(.035)) / 2))$

\#\# [1] 0.9860377

print(corr.test(pp.full\$withdrawal.s, pp.full\$withdrawal.i, method = "spearman"), short $=\mathrm{F}$, digits $=3$ )

\#\# Call:corr.test $(\mathrm{x}=$ pp.full\$withdrawal.s, $\mathrm{y}=$ pp.full\$withdrawal.i,

\#\# $\quad$ method $=$ "spearman")

\#\# Correlation matrix

\#\# [1] 0.328

\#\# Sample Size

\#\# [1] 208

\#\# Probability values adjusted for multiple tests.

\#\# [1] 0

\#\#

\#\# Confidence intervals based upon normal theory. To get bootstrapped values, try cor.ci

\#\# raw.lower raw.r raw.upper raw.p lower.adj upper.adj

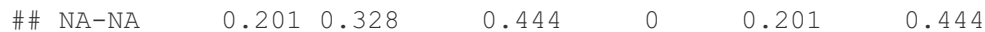

\# Base model

pp.full\$withdrawal.s_c $=$ pp.full\$withdrawal.s - mean(pp.full\$withdrawal.s)

pp.full\$withdrawal.i_c $=$ pp.full\$withdrawal.i - mean(pp.full\$withdrawal.s)

wit1 $=$ lm(withdrawal.i_c $~ 1+$ withdrawal.s_c, data $=$ pp.full)

summary (wit1)

\#\#

\#\# Call:

\#\# $\operatorname{lm}$ (formula $=$ withdrawal.i_c $\sim 1+$ withdrawal.s_c, data $=$ pp.full)

\#\#

\#\# Residuals:

\#\# Min 10 Median $30 \quad$ Max

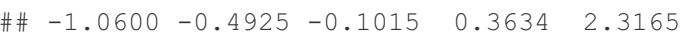

\#\#

\#\# Coefficients:

\#\# Estimate Std. Error $t$ value $\operatorname{Pr}(>|t|)$

$\begin{array}{lllll}\text { \#\# (Intercept) } & 0.02604 & 0.04120 & 0.632 & 0.528\end{array}$

\#\# withdrawal.s_c $0.29780 \quad 0.06287 \quad 4.737 \quad 4.03 e-06 * * *$

\#\#---

\#\# Signif. codes: 0 '***' 0.001 '**' $0.011^{\prime *} 0.05$ '.' 0.1 ' ' 1

\#\#

\#\# Residual standard error: 0.5942 on 206 degrees of freedom

\#\# Multiple R-squared: 0.09823, Adjusted R-squared: 0.09386

\#\# F-statistic: 22.44 on 1 and 206 DF, p-value: $4.032 \mathrm{e}-06$

round(confint(wit1), 3)

$\begin{array}{lrrr}\text { \#\# } & 2.5 \% 97.5 \% \\ \text { \#\# (Intercept) } & -0.055 & 0.107 \\ \text { \#\# withdrawal.s_c } & 0.174 & 0.422\end{array}$

\# Moderation model

wit2 $=$ lm(withdrawal.i_c $\sim$ withdrawal.s_c*gen.z, data $=$ pp.full)

summary (wit2) 
\#\# Call:

\#\# $\operatorname{lm}($ formula $=$ withdrawal.i $c \sim$ withdrawal.s $c *$ gen.z, data $=$ pp.full)

\#\#

\#\# Residuals:

\#\# $\quad$ Min $\quad 10 \quad$ Median $\quad 30 \quad$ Max

$\begin{array}{llllll}\# \# & -1.40123 & -0.35987 & -0.07066 & 0.35348 & 2.17278\end{array}$

\#\#

\#\# Coefficients:

\#\# Estimate Std. Error t value Pr $(>|t|)$

$\begin{array}{lllll}\text { \#\# (Intercept) } & 0.04859 & 0.04116 & 1.180 & 0.239\end{array}$

$\begin{array}{lllll}\text { \#\# withdrawal.s_c } & 0.07696 & 0.06881 & 1.118 & 0.265\end{array}$

$\begin{array}{llllll}\text { \# } \text { gen.z } & 0.30513 & 0.04417 & 6.908 & 6.12 e-11 * * *\end{array}$

\#\# withdrawal.s_c:gen.z $-0.06522 \quad 0.05073 \quad-1.286 \quad 0.200$

$\# \#---$

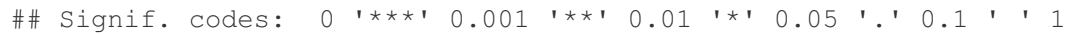

\#\#

\#\# Residual standard error: 0.5371 on 204 degrees of freedom

\#\# Multiple R-squared: 0.2704, Adjusted R-squared: 0.2597

\#\# F-statistic: 25.2 on 3 and 204 DF, p-value: $6.508 e-14$

round (confint (wit2), 3)

$\begin{array}{rrr}\text { \#\# } & 2.5 \% & 97.5 \% \\ \# \# \text { (Intercept) } & -0.033 & 0.130 \\ \text { \#\# withdrawal.s_c } & -0.059 & 0.213 \\ \text { \#\# gen.z } & 0.218 & 0.392 \\ \text { \#\# withdrawal.s_c:gen.z } & -0.165 & 0.035\end{array}$

anova (wit1, wit2)

\#\# Analysis of Variance Table

\#\#

\#\# Model 1: withdrawal.i_c 1 + withdrawal.s_c

\#\# Model 2: withdrawal.i_c $\sim$ withdrawal.s_c * gen.z

\#\# Res.Df RSS Df Sum of Sq F $\operatorname{Pr}(>\mathrm{F})$

\#\# $1206 \quad 72.733$

\#\# $220458.8462 \quad 13.888 \quad 24.072 \quad 4.111 e-10 * * *$

\#\# ---

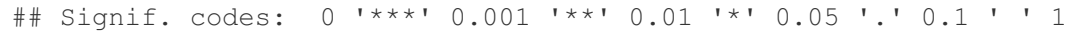

\section{Self-other agreement for PID-5 PD scores}

\#\#\# Correlational accuracy and mean-level bias \#\#\#\#\#

\#\# Antisocial PD

t.test (pp.full\$anti.pid.s, pp.full\$anti.pid.i, paired $=T$ )

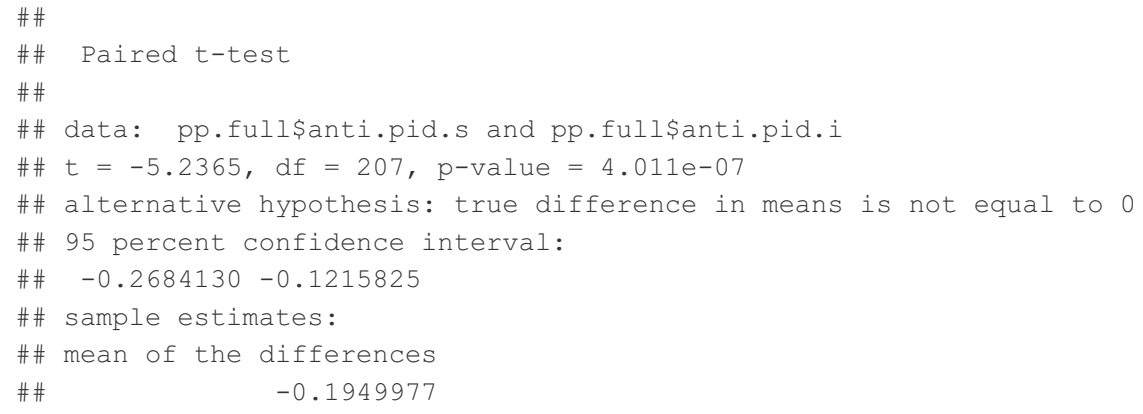


\#\# Wilcoxon signed rank test with continuity correction

\#\# data: pp.full\$anti.pid.i and pp.full\$anti.pid.s

\#\# $\mathrm{V}=14538, \mathrm{p}$-value $=1.227 \mathrm{e}-05$

\#\# alternative hypothesis: true location shift is not equal to 0

\section{$(5.2365 / \operatorname{sqrt}(208))$}

\#\# [1] 0.3630859

$\operatorname{pnorm}(.363)$

\#\# [1] 0.6416976

$(2 * \operatorname{pnorm}((-\operatorname{abs}(.363)) / 2))$

\#\# [1] 0.8559751

print(corr.test (pp.full\$anti.pid.s, pp.full\$anti.pid.i, method = "spearman"), short = F, digits = 3)

\#\# Call:corr.test ( $x=$ pp.full\$anti.pid.s, $y=$ pp.full\$anti.pid.i, method = "spearman")

\#\# Correlation matrix

\#\# [1] 0.414

\#\# Sample Size

\#\# [1] 208

\#\# Probability values adjusted for multiple tests.

\#\# [1] 0

\#\#

\# Confidence intervals based upon normal theory. To get bootstrapped values, try cor.ci

\#\# raw.lower raw.r raw.upper raw.p lower.adj upper.adj

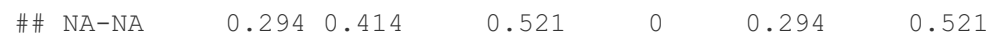

\#\# Avoidant PD

t.test (pp.full\$avoid.pid.s, pp.full\$avoid.pid.i, paired $=T$ )

\#\#

\#\# Paired t-test

\#\#

\#\# data: pp.full\$avoid.pid.s and pp.full\$avoid.pid.i

$\# \#=-0.86104, \mathrm{df}=207, \mathrm{p}$-value $=0.3902$

\#\# alternative hypothesis: true difference in means is not equal to 0

\#\# 95 percent confidence interval:

\#\# -0.1121254 0.0439573

\#\# sample estimates:

\#\# mean of the differences

\#\# $\quad-0.03408406$

wilcox.test (pp.full\$avoid.pid.s, pp.full\$avoid.pid.i, paired $=\mathrm{T}$ )

\#\#

\#\# Wilcoxon signed rank test with continuity correction

\#\#

\#\# data: pp.full\$avoid.pid.s and pp.full\$avoid.pid.i

\#\# $\mathrm{V}=10238, \mathrm{p}$-value $=0.4685$

\#\# alternative hypothesis: true location shift is not equal to 0

$(.86104 / \operatorname{sqrt}(208))$ 
\#\# [1] 0.5239222

$(2 * \operatorname{pnorm}((-\operatorname{abs}(.060)) / 2))$

\#\# [1] 0.9760671

print (corr.test (pp.full\$avoid.pid.s, pp.full\$avoid.pid.i, method = "spearman"), short = F, digits = 3)

\#\# Call:corr.test $(x=$ pp.full\$avoid.pid.s, $y=$ pp.full\$avoid.pid.i, method = "spearman")

\#\# Correlation matrix

\#\# [1] 0.432

\#\# Sample Size

\#\# [1] 208

\#\# Probability values adjusted for multiple tests.

\#\# [1] 0

\#\#

\#\# Confidence intervals based upon normal theory. To get bootstrapped values, try cor.ci

\#\# raw.lower raw.r raw.upper raw.p lower.adj upper.adj

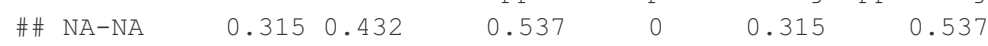

\#\# BPD

t.test (pp.full\$bord.pid.s, pp.full\$bord.pid.i, paired $=T$ )

\#\#

\#\# Paired t-test

\#\#

\#\# data: pp.full\$bord.pid.s and pp.full\$bord.pid.i

\#\# $t=-3.4406, \mathrm{df}=207, \mathrm{p}$-value $=0.0007019$

\#\# alternative hypothesis: true difference in means is not equal to 0

\#\# 95 percent confidence interval:

\#\# -0.19630294-0.05328695

\#\# sample estimates:

\#\# mean of the differences

\#\#

$-0.1247949$

wilcox.test (pp.full\$bord.pid.s, pp.full\$bord.pid.i, paired $=T$ )

\#\#

\#\# Wilcoxon signed rank test with continuity correction

\#\#

\#\# data: pp.full\$bord.pid.s and pp.full\$bord.pid.i

\#\# $\mathrm{V}=8403, \mathrm{p}$-value $=0.004572$

\#\# alternative hypothesis: true location shift is not equal to 0

$(3.4406 /$ sqrt (208))

\# [1] 0.2385627

pnorm (.239)

\#\# [1] 0.5944472

$(2 * \operatorname{pnorm}((-\mathrm{abs}(.239)) / 2))$

\#\# [1] 0.9048792

print(corr.test (pp.full\$bord.pid.s, pp.full\$bord.pid.i, method = "spearman"), short = F, digits = 3) 
\#\# Call:corr.test ( $x=$ pp.full\$bord.pid.s, $y=$ pp.full\$bord.pid.i, method = "spearman")

\#\# Correlation matrix

\#\# [1] 0.488

\#\# Sample Size

\#\# [1] 208

\#\# Probability values adjusted for multiple tests.

\#\# [1] 0

\#\#

\#\# Confidence intervals based upon normal theory. To get bootstrapped values, try cor.ci

\#\# raw.lower raw.r raw.upper raw.p lower.adj upper.adj

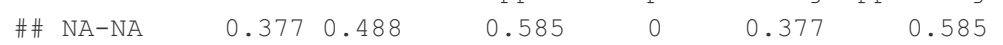

\#\# Narcissistic PD

t.test(pp.full\$nar.pid.s, pp.full\$nar.pid.i, paired $=\mathrm{T}$ )

\section{\#\#}

\#\# Paired t-test

\#\#

\#\# data: pp.full\$nar.pid.s and pp.full\$nar.pid.i

\#\# $\mathrm{t}=-3.1586, \mathrm{df}=207, \mathrm{p}$-value $=0.001822$

\#\# alternative hypothesis: true difference in means is not equal to 0

\#\# 95 percent confidence interval:

\#\# -0.2707549-0.0626528

\#\# sample estimates:

\#\# mean of the differences

\#\#

$-0.1667039$

wilcox.test (pp.full\$nar.pid.s, pp.full\$nar.pid.i, paired = T)

\#\#

\#\# Wilcoxon signed rank test with continuity correction

\#\#

\#\# data: pp.full\$nar.pid.s and pp.full\$nar.pid.i

\#\# $\mathrm{V}=7729$, p-value $=0.004633$

\#\# alternative hypothesis: true location shift is not equal to 0

$(3.1586 / \operatorname{sqrt}(208))$

\#\# [1] 0.2190095

$\operatorname{pnorm}(.219)$

\#\# [1] 0.586675

$(2 * \operatorname{pnorm}((-\operatorname{abs}(.219)) / 2))$

\#\# [1] 0.9128059

print(corr.test (pp.full\$nar.pid.s, pp.full\$nar.pid.i, method = "spearman"), short = F, digits = 3)

\#\# Call:corr.test $(x=p p . f u l l \$ n a r . p i d . s, y=p p . f u l l \$ n a r . p i d . i$, method = "spearman")

\#\# Correlation matrix

\#\# [1] 0.295

\#\# Sample Size

\#\# [1] 208

\#\# Probability values adjusted for multiple tests.

\#\# [1] 0

\#\#

\# Confidence intervals based upon normal theory. To get bootstrapped values, try cor.ci

\#\# raw.lower raw.r raw.upper raw.p lower.adj upper.adj

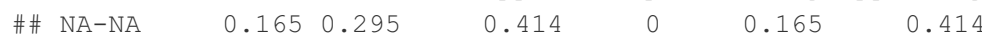




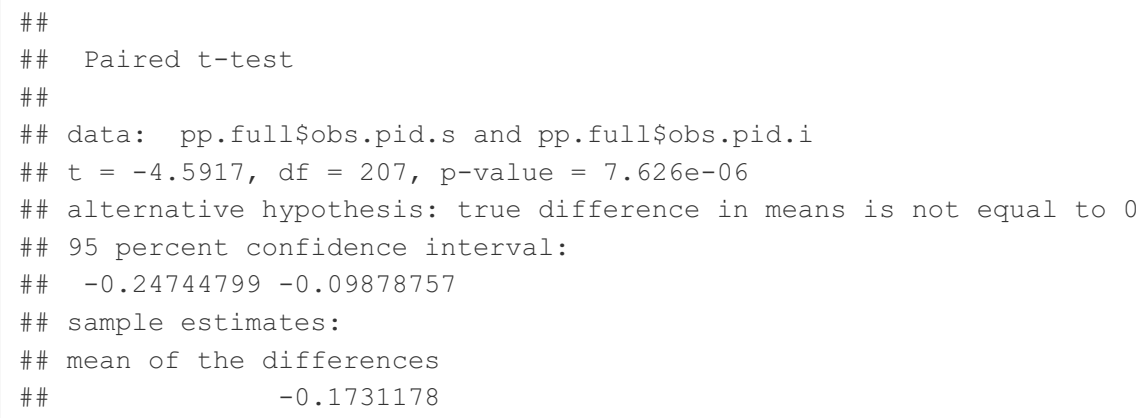

\#\# [1] 0.3183771

$\operatorname{pnorm}(.318)$

\#\# [1] 0.6247575

$(2 * \operatorname{pnorm}((-\mathrm{abs}(.318)) / 2))$

\#\# [1] 0.8736689

print(corr.test(pp.full\$obs.pid.s, pp.full\$obs.pid.i, method = "spearman"), short = F, digits = 3)

\#\# Call:corr.test ( $\mathrm{x}=\mathrm{pp}$.full\$obs.pid.s, $\mathrm{y}=$ pp.full\$obs.pid.i, method = "spearman")

\#\# Correlation matrix

\#\# [1] 0.32

\#\# Sample Size

\#\# [1] 208

\#\# Probability values adjusted for multiple tests.

\#\# [1] 0

\#\#

\#\# Confidence intervals based upon normal theory. To get bootstrapped values, try cor.ci

\#\# raw.lower raw.r raw.upper raw.p lower.adj upper.adj

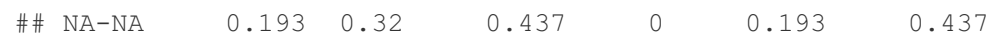

\#\# Schizotypal PD

t.test (pp.full\$schiz.pid.s, pp.full\$schiz.pid.i, paired $=T$ ) 


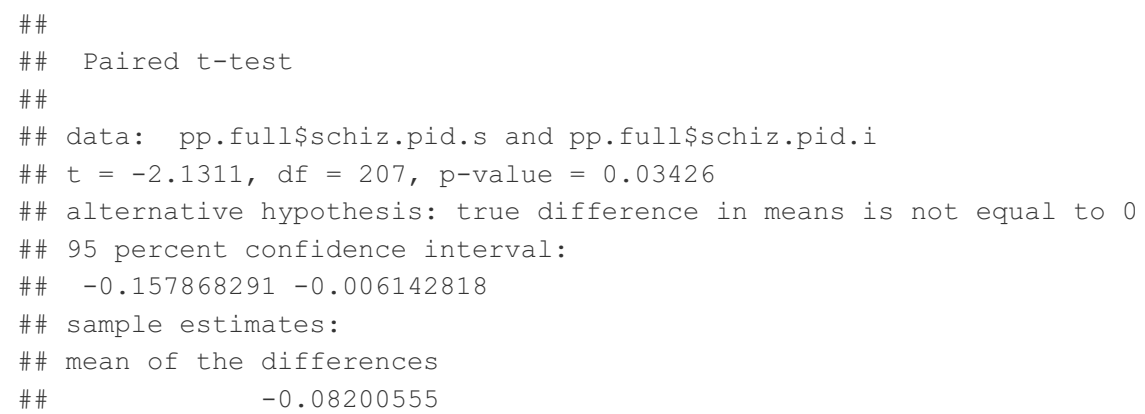

\# \# [1] 0.1477652

$\operatorname{pnorm}(.148)$

\#\# [1] 0.5588286

$(2 * \operatorname{pnorm}((-\operatorname{abs}(.148)) / 2))$

\#\# [1] 0.9410104

print (corr.test (pp.full\$schiz.pid.s, pp.full\$schiz.pid.i, method = "spearman"), short = F, digits = 3)

\#\# Call:corr.test $(x=$ pp.full\$schiz.pid.s, $y=$ pp.full\$schiz.pid.i, method = "spearman")

\#\# Correlation matrix

\#\# [1] 0.295

\#\# Sample Size

\#\# [1] 208

\#\# Probability values adjusted for multiple tests.

\#\# [1] 0

\#\#

\#\# Confidence intervals based upon normal theory. To get bootstrapped values, try cor.ci

\#\# raw.lower raw.r raw.upper raw.p lower.adj upper.adj
\#\# NA-NA
0.1660 .295
0.415
0.166
0.415

\#\#\#\#\#\# Regression models \#\#\#\#\#

\#\#\#\# Antisocial PD \#\#\#\#\#\#\#\#

\# Base model

pp.full\$anti.pid.s c = pp.full\$anti.pid.s - mean(pp.full\$anti.pid.s)

pp.full\$anti.pid.i_c $=$ pp.full\$anti.pid.i - mean (pp.full\$anti.pid.s)

anti.modell = lm(anti.pid.i $c \sim 1+$ anti.pid.s c, data = pp.full)

summary (anti.model1) 
\#\# Call:

\#\# $\operatorname{lm}$ (formula $=$ anti.pid.i $c \sim 1+$ anti.pid.s c, data $=$ pp.full)

\#\#

\#\# Residuals:

\#\# Min $\quad 10$ Median $\quad 30 \quad$ Max

$\begin{array}{llllll}\# \#-1.01143 & -0.37343 & -0.09709 & 0.32756 & 1.65626\end{array}$

\#\#

\#\# Coefficients:

\#\# Estimate Std. Error $t$ value $\operatorname{Pr}(>|t|)$

\#\# (Intercept) $0.19500 \quad 0.03486 \quad 5.5937 .04 \mathrm{e}-08 * * *$

\#\# anti.pid.s_c $0.57047 \quad 0.07821 \quad 7.294 \quad 6.38 \mathrm{e}-12 * \star *$

\#\#---

\#\# Signif. codes: 0 '***' $0.001, * * \prime 0.01$ '*' 0.05 '.'0.1' 0.1

\#\#

\#\# Residual standard error: 0.5028 on 206 degrees of freedom

\#\# Multiple R-squared: 0.2052, Adjusted R-squared: 0.2014

\#\# F-statistic: 53.2 on 1 and 206 DF, p-value: 6.376e-12

round (confint (anti.model1), 3)

$\begin{array}{llrr}\text { \#\# } & 2.5 \% & 97.5 \% \\ \text { \#\# } & \text { (Intercept) } & 0.126 & 0.264 \\ \text { \#\# } & \text { anti.pid.s_c } & 0.416 & 0.725\end{array}$

\# Moderation model

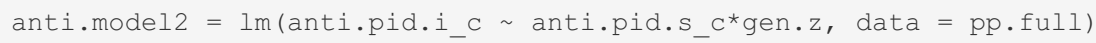

summary (anti.model2)

\#\#

\#\# Call:

\#\# $\operatorname{lm}$ (formula $=$ anti.pid.i $c \sim \operatorname{anti.pid.s~} \mathrm{c} \star$ gen.z, data $=$ pp.full)

\#\#

\#\# Residuals:

\#\# Min 10 Median $30 \quad$ Max

$\begin{array}{llllll}\# \# & -1.02127 & -0.34906 & -0.08503 & 0.29717 & 1.61816\end{array}$

\#\#

\#\# Coefficients:

\#\# Estimate Std. Error $t$ value Pr $(>|t|)$

\#\# (Intercept) $0.19760 \quad 0.03501 \quad 5.6435 .53 e-08 * * *$

\#\# anti.pid.s_c $\quad 0.35819 \quad 0.08712 \quad 4.1125 .69 e-05 * * *$

\#\# gen.z $\quad 0.21049 \quad 0.03705 \quad 5.6814 .57 e-08 * * *$

$\begin{array}{llllll}\text { \# } \text { anti.pid.s_c:gen.z } & -0.01259 & 0.06258 & -0.201 & 0.841\end{array}$

\#\#---

\#\# Signif. codes: 0 '***' 0.001 '**' 0.01 '*' 0.05 '.' 0.1 ' ' 1

\#\#

\#\# Residual standard error: 0.4693 on 204 degrees of freedom

\#\# Multiple R-squared: 0.3143, Adjusted R-squared: 0.3042

\#\# F-statistic: 31.17 on 3 and 204 DF, p-value: < 2.2e-16

round (confint (anti.model2), 3)

$\begin{array}{lrr}\text { \#\# } & 2.5 \% & 97.5 \% \\ \text { \#\# (Intercept) } & 0.129 & 0.267 \\ \text { \#\# anti.pid.s_c } & 0.186 & 0.530 \\ \text { \#\# gen.z } & 0.137 & 0.284 \\ \text { \#\# anti.pid.s_c:gen.z } & -0.136 & 0.111\end{array}$

anova (anti.model1, anti.model2) 


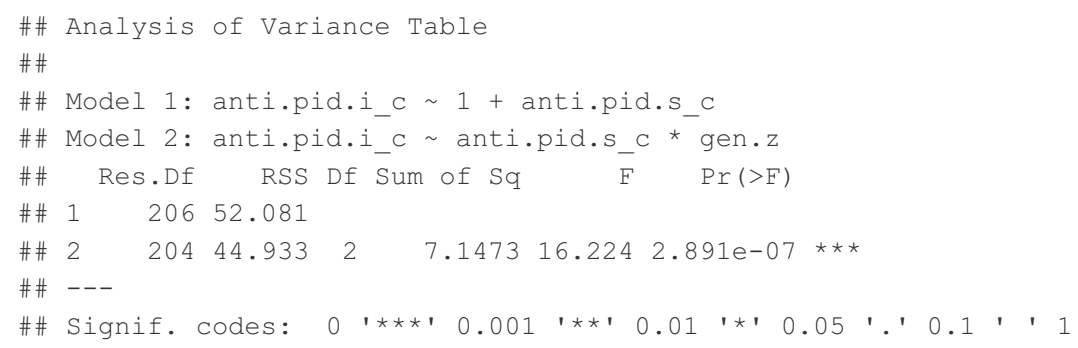

$\begin{array}{rrrr}\text { \#\# } & 2.5 \% & 97.5 \% \\ \text { \#\# (Intercept) } & -0.031 & 0.099 \\ \text { \#\# avoid.pid.s C } & 0.299 & 0.534\end{array}$


avoid.model2 = lm(avoid.pid.i_c $\sim$ avoid.pid.s_c*gen.z, data $=$ pp.full)

summary (avoid.model2)

\section{\#\#}

\#\# Call:

\#\# $\operatorname{lm}($ formula = avoid.pid.i_c $\sim$ avoid.pid.s_c * gen.z, data $=$ pp.full)

\#\#

\#\# Residuals:

\#\# Min $\quad 1 Q$ Median $3 Q \quad$ Max

$\begin{array}{llllll}\# \# & -0.89948 & -0.30717 & -0.02707 & 0.29532 & 1.06143\end{array}$

\#\#

\#\# Coefficients:

\#\#

\#\# (Intercept)

Estimate std. Error $t$ value $\operatorname{Pr}(>|t|)$

$\begin{array}{lllll}\text { \#\# avoid.pid.s_c } & 0.08722 & 0.06909 & 1.262 & 0.208\end{array}$

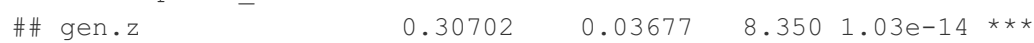

$\begin{array}{lllll}\text { \# } \# \text { avoid.pid.s c:gen.z } & -0.04437 & 0.04431 & -1.001 & 0.318\end{array}$

\#\# ---

\#\# Signif. codes: 0 '***' 0.001 '**' 0.01 '*' 0.05 '.' 0.1 ' ' 1

\#\#

\#\# Residual standard error: 0.4102 on 204 degrees of freedom

\#\# Multiple R-squared: 0.3978, Adjusted R-squared: 0.389

\#\# F-statistic: 44.93 on 3 and 204 DF, p-value: < 2.2e-16

round (confint (avoid.model2), 3)

$\begin{array}{lrr}\text { \#\# } & 2.5 \% & 97.5 \% \\ \# \# \text { (Intercept) } & -0.014 & 0.113 \\ \text { \#\# avoid.pid.s_c } & -0.049 & 0.223 \\ \text { \#\# gen.z } & 0.235 & 0.380 \\ \text { \#\# avoid.pid.s_c:gen.z } & -0.132 & 0.043\end{array}$

anova (avoid.model1, avoid.model2) \#p<.001

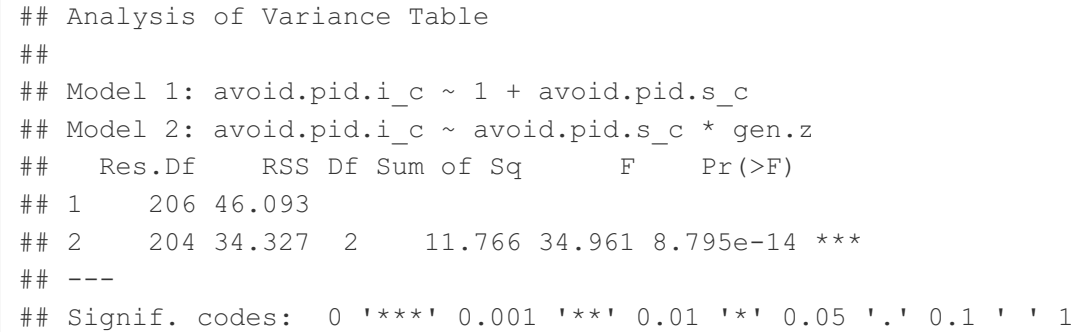




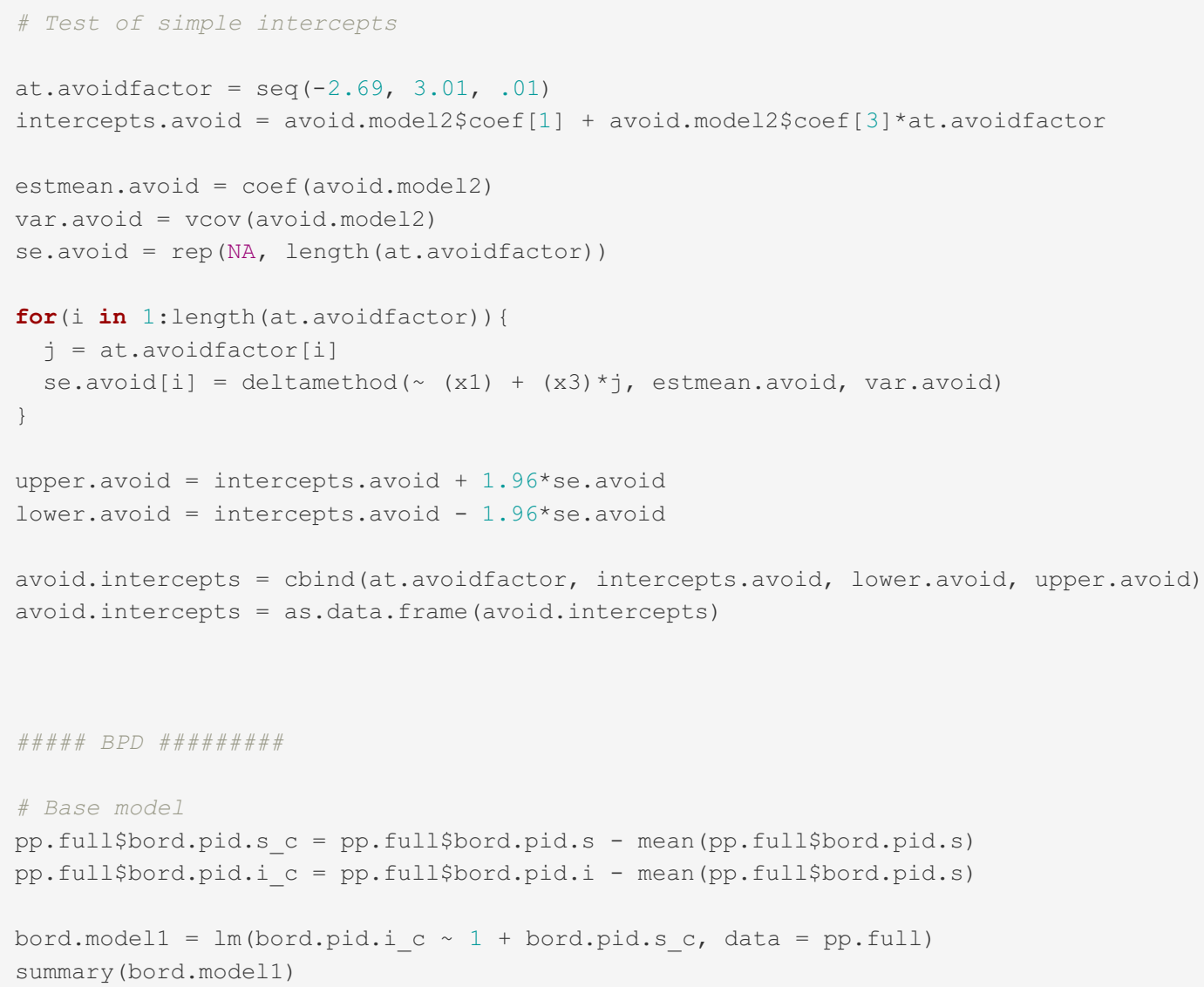

$\begin{array}{llrr}\text { \#\# } & 2.5 \div 97.5 \% \\ \text { \#\# (Intercept) } & 0.060 & 0.189 \\ \text { \#\# bord.pid.s_c } & 0.418 & 0.675\end{array}$

\#\# Moderation model

bord.model2 $=\operatorname{lm}($ bord.pid.i_c $\sim$ bord.pid.s_c*gen.z, data $=$ pp.full) summary (bord.model2) 
\#\# Call:

\#\# $\operatorname{lm}$ (formula $=$ bord.pid.i $\mathrm{c} \sim$ bord.pid.s $\mathrm{c} *$ gen.z, data $=$ pp.full)

\#\#

\#\# Residuals:

\#\# Min 12 Median $30 \quad$ Max

$\begin{array}{llllll}\# \# & -0.88776 & -0.32243 & -0.08532 & 0.29390 & 1.44777\end{array}$

\#\#

\#\# Coefficients:

\#\# Estimate Std. Error $t$ value $\operatorname{Pr}(>|t|)$

$\begin{array}{llllll}\text { \# } \text { (Intercept) } \quad 0.123321 & 0.033422 & 3.690 & 0.000288 * * *\end{array}$

\#\# bord.pid.s_c $\quad 0.316523 \quad 0.070464 \quad 4.4921 .18 e-05 * * *$

\#\# gen.z $0.225724 \quad 0.035271 \quad 6.4001 .05 e-09 * * *$

$\begin{array}{lllll}\text { \#\# bord.pid.s_c:gen.z } & 0.005792 & 0.058424 & 0.099 & 0.921131\end{array}$

\#\#---

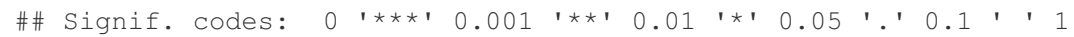

\#\#

\#\# Residual standard error: 0.4317 on 204 degrees of freedom

\#\# Multiple R-squared: 0.382, Adjusted R-squared: 0.373

\#\# F-statistic: 42.04 on 3 and 204 DF, p-value: $<2.2 e-16$

round (confint (bord.model2), 3)

\begin{tabular}{lrr}
\hline$\# \#$ & $2.5 \div$ & $97.5 \%$ \\
$\# \#$ (Intercept) & 0.057 & 0.189 \\
$\# \#$ bord.pid.s_c & 0.178 & 0.455 \\
\#\# gen.z & 0.156 & 0.295 \\
\#\# bord.pid.s_c:gen.z & -0.109 & 0.121 \\
\hline
\end{tabular}

anova (bord.model1, bord.model2)

\#\# Analysis of Variance Table

\#\#

\#\# Model 1: bord.pid.i_c 1 + bord.pid.s_c

\#\# Model 2: bord.pid.i_c bord.pid.s_c * gen.z

\#\# Res.Df RSS Df Sum of Sq F $\operatorname{Pr}(>\mathrm{F})$

\#\# $1 \quad 206 \quad 45.875$

\#\# $2 \quad 204 \quad 38.0152 \quad 7.8594 \quad 21.088 \quad 4.735 e-09 * \star *$

\#\#---

\#\# Signif. codes: 0 '***' 0.001 '**' 0.01 '*' 0.05 '.' 0.1 ' ' 1 


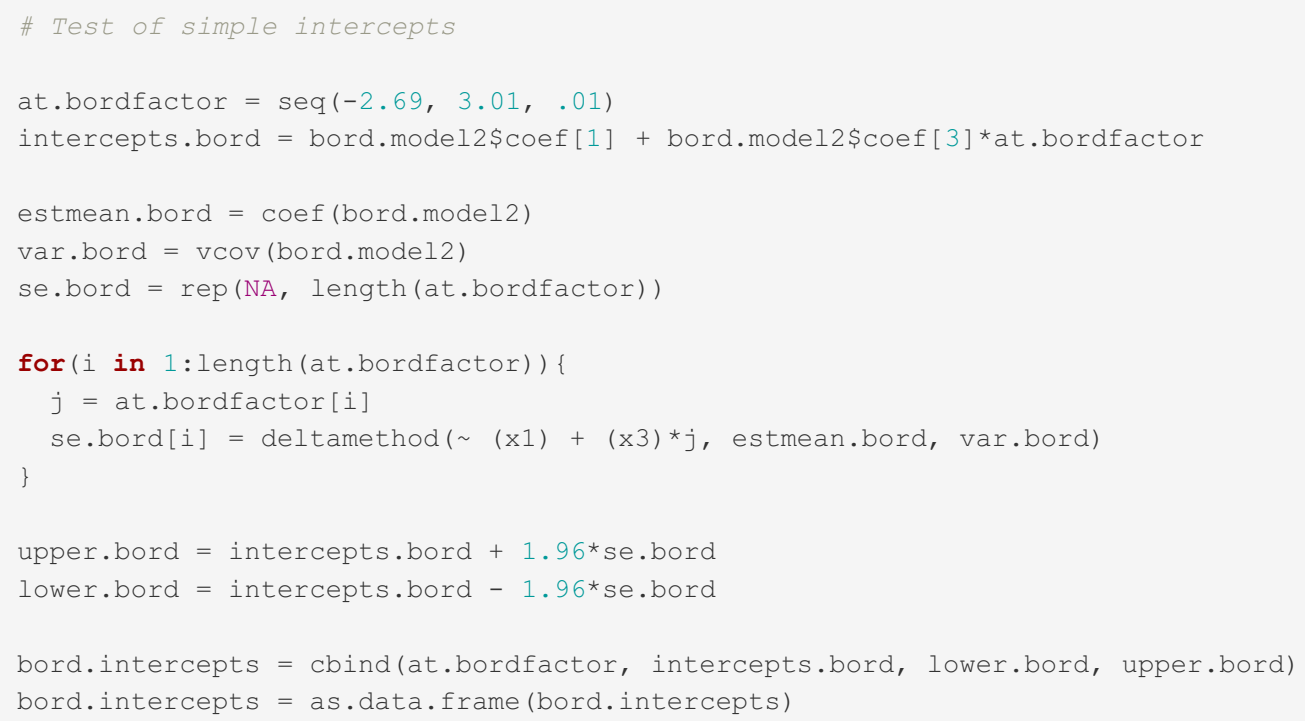

round (confint (nar.model1), 3)

$\begin{array}{lrrr}\text { \#\# } & 2.5 \% & 97.5 \% \\ \text { \#\# } & \text { (Intercept) } & 0.075 & 0.259 \\ \text { \#\# } & \text { nar.pid.s_c } & 0.205 & 0.530\end{array}$

* Moderation model

nar.model $2=\operatorname{lm}($ nar.pid.i_c $\sim$ nar.pid.s_c*gen.z, data $=$ pp.full)

summary (nar.model2) 
\#\# Call:

\#\# $\operatorname{lm}($ formula $=$ nar.pid.i_c $\sim$ nar.pid.s_c $*$ gen.z, data $=$ pp.full)

\#\#

\#\# Residuals:

\# Min 10 Median $30 \quad$ Max

$\begin{array}{llllll}\# \# & -1.1763 & -0.4547 & -0.1037 & 0.4673 & 2.2013\end{array}$

\#\#

\#\# Coefficients:

\#\# Estimate std. Error $t$ value $\operatorname{Pr}(>|t|)$

\#\# (Intercept) $0.16173 \quad 0.04583 \quad 3.5290 .000516 * * *$

\#\# nar.pid.s_c $\quad 0.28496 \quad 0.08189 \quad 3.480 \quad 0.000614 * * *$

\#\# gen.z $0.19583 \quad 0.04625 \quad 4.234 \quad 3.47 e-05 * * *$

$\begin{array}{lllll}\text { \#\# nar.pid.s_c:gen.z } & 0.03974 & 0.07428 & 0.535 & 0.593239\end{array}$

\#\#---

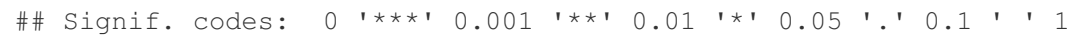

\#\#

\#\# Residual standard error: 0.6473 on 204 degrees of freedom

\#\# Multiple R-squared: 0.1641, Adjusted R-squared: 0.1518

\#\# F-statistic: 13.35 on 3 and 204 DF, p-value: 5.469e-08

round (confint (nar.model2), 3)

\begin{tabular}{|rrr}
\hline$\# \#$ & $2.5 \div$ & $97.5 \%$ \\
$\# \#$ (Intercept) & 0.071 & 0.252 \\
$\# \#$ nar.pid.s_c & 0.123 & 0.446 \\
$\# \#$ gen.z & 0.105 & 0.287 \\
$\# \#$ nar.pid.s_c:gen.z & -0.107 & 0.186 \\
\hline
\end{tabular}

anova (nar.model1, nar.model2)

\#\# Analysis of Variance Table

\#\#

\#\# Model 1: nar.pid.i c $\sim 1+$ nar.pid.s c

\#\# Model 2: nar.pid.i_c nar.pid.s_c * gen.z

\#\# Res.Df RSS Df Sum of Sq F $\operatorname{Pr}(>\mathrm{F})$

\#\# $1206 \quad 93.275$

$\begin{array}{llllllll}\text { \#\# } 2 & 204 & 85.475 & 2 & 7.8004 & 9.3085 & 0.0001353 * * *\end{array}$

\#\#---

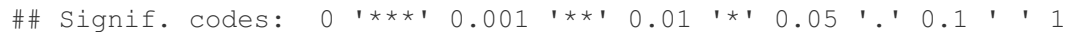




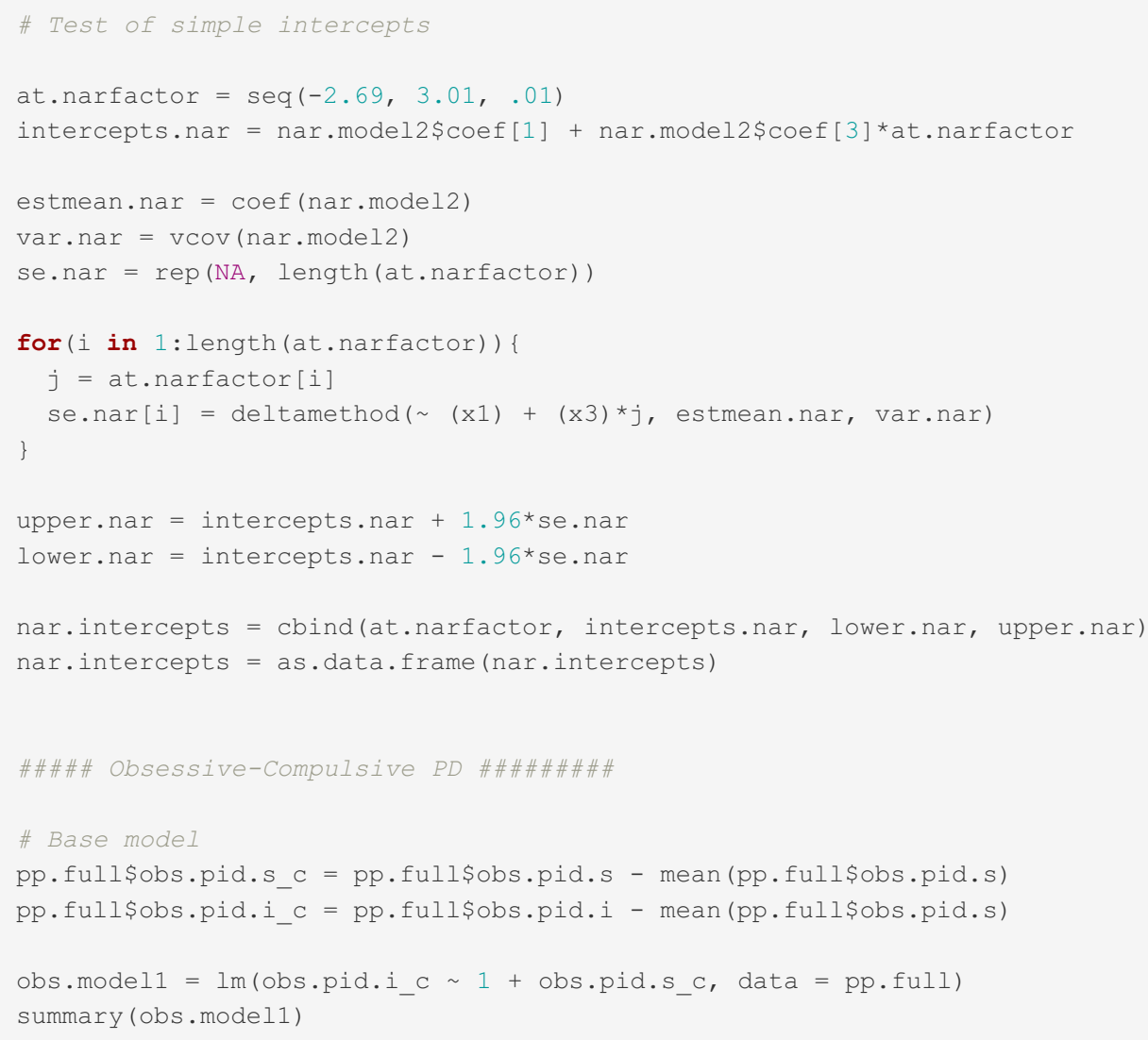

round (confint (obs.model1), 3)

\begin{tabular}{|c|c|}
\hline \#\# & $2.5 \div 97.5 \%$ \\
\hline \#\# (Intercept) & $0.113 \quad 0.234$ \\
\hline \#\# obs.pid.s_c & 0.195 \\
\hline
\end{tabular}

\#\# Moderation model

obs.model2 $=$ lm(obs.pid.i_c $\sim$ obs.pid.s_c*gen.z, data $=$ pp.full) summary (obs.model2) 
\#\# Call:

\#\# $\operatorname{lm}$ (formula $=$ obs.pid.i_c $\sim$ obs.pid.s_c $*$ gen.z, data $=$ pp.full)

\#\#

\#\# Residuals:

\#\# Min 10 Median $30 \quad$ Max

$\begin{array}{llllll}\# \# & -0.92879 & -0.32664 & -0.01474 & 0.28158 & 1.06063\end{array}$

\#\#

\#\# Coefficients:

\#\# Estimate std. Error $t$ value $\operatorname{Pr}(>|t|)$

\#\# (Intercept) $0.18235 \quad 0.03062 \quad 5.9561 .12 \mathrm{e}-08 * * *$

$\begin{array}{lllll}\text { \#\# obs.pid.s_c } & 0.12245 & 0.06965 & 1.758 & 0.0802\end{array}$

\#\# gen.z $\quad 0.20677 \quad 0.03265 \quad 6.334 \quad 1.50 e-09 * \star *$

$\begin{array}{llllll}\text { \#\# obs.pid.s_c:gen.z } & -0.04076 & 0.05264 & -0.774 & 0.4396\end{array}$

\#\#---

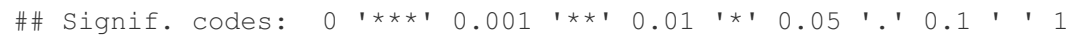

\#\#

\#\# Residual standard error: 0.4067 on 204 degrees of freedom

\#\# Multiple R-squared: 0.2531, Adjusted R-squared: 0.2421

\#\# F-statistic: 23.04 on 3 and 204 DF, p-value: 6.892e-13

round (confint (obs.model2), 3)

\begin{tabular}{|rrr|}
\hline$\# \#$ & $2.5 \div$ & $97.5 \%$ \\
$\# \#$ (Intercept) & 0.122 & 0.243 \\
$\# \#$ obs.pid.s_c & -0.015 & 0.260 \\
$\# \#$ gen.z & 0.142 & 0.271 \\
$\# \#$ obs.pid.s_c:gen.z & -0.145 & 0.063 \\
\hline
\end{tabular}

anova (obs.model1, obs.model2)

\#\# Analysis of Variance Table

\#\#

\#\# Model 1: obs.pid.i_c $\sim 1+$ obs.pid.s_c

\#\# Model 2: obs.pid.i_c obs.pid.s_c * gen.z

\#\# Res.Df RSS Df Sum of Sq F $\operatorname{Pr}(>\mathrm{F})$

\#\# $1 \quad 206 \quad 40.387$

\#\# $2 \quad 204 \quad 33.747 \quad 2 \quad 6.639120 .066 \quad 1.108 e-08$ ***

\#\#---

\#\# Signif. codes: 0 '***' 0.001 '**' 0.01 '*' 0.05 '.' 0.1 ' ' 1 


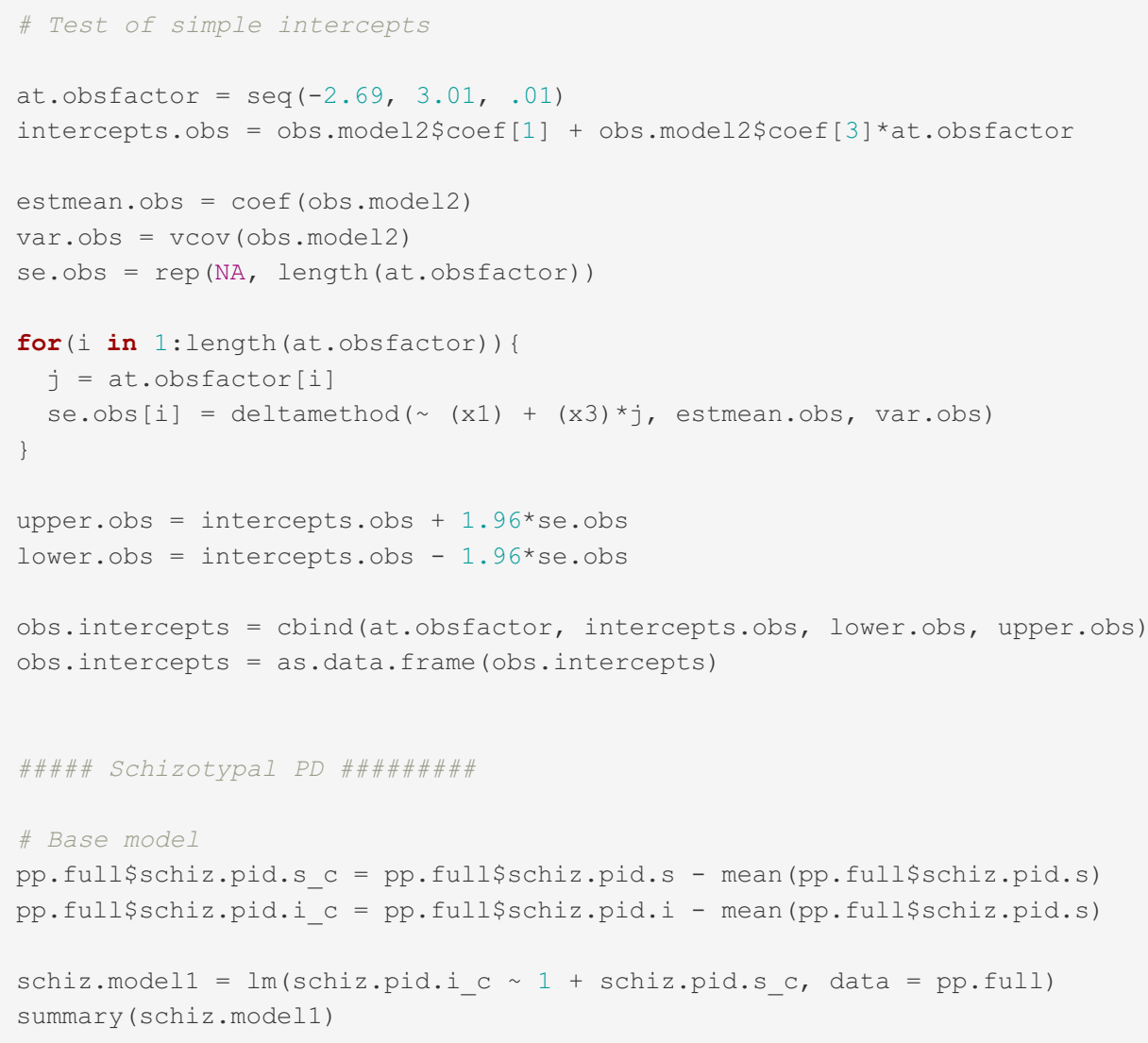

\begin{tabular}{|c|c|}
\hline \#\# & $2.5 \div 97.5 \%$ \\
\hline \#\# (Intercept) & $0.022 \quad 0.142$ \\
\hline \#\# schiz.pid.s_c & 0.146 \\
\hline
\end{tabular}

\# Moderation model

schiz.model2 = lm(schiz.pid.i $c \sim$ schiz.pid.s $c^{\star} g e n . z$, data $=$ pp.full)

summary (schiz.model2) 
domain.p $=\mathrm{c}(.08223, \# \mathrm{dz}$ for $N A$

$1.377969 \mathrm{e}-11$, \# r for $N A$

$.0005768, \# d z$ for $A T$

9.17164e-05, \# r for AT

7.377e-09, \# dz for DS

$5.029261 e-19$, \# r for DS

$.4285, \# d z$ for PS

$5.1054 e-06$, \# r for PS

$0.1097, \# d z$ for DT

.0006405343 , \# r for DT

0.0469 , \# bo for base model of NA

3.04e-11, \# b1 for base model of NA

0.052032 , \# bo for moderation model of NA

0.000206 , \# b1 for moderation model of NA

3.97e-10, \# b2 for moderation model of NA

0.945073 , \# b3 for moderation model of NA

$0.000158,1.22 e-05,0.000253,0.030118,4.25 e-08,0.428927$, \# AT models

$4.03 e-10,2 e-16,6.90 e-11,7.01 e-15,0.00087,0.10420$, \# DS models

$0.321489,0.000405,0.1476,0.0181,4.23 e-07,0.1475$, \# PS models

$0.0515,1.61 e-09,0.0134,0.0830,3.76 e-11,0.2181)$ \# DT models

domain.p.adjust $=$ p.adjust (domain.p, method = "BH", $\mathrm{n}=$ length (domain.p))

round (domain.p.adjust, 3)

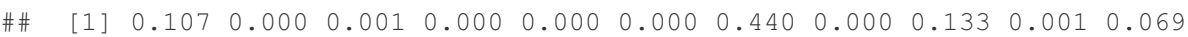

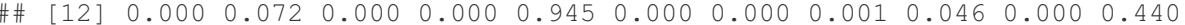

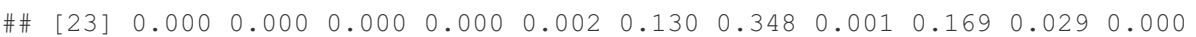

$\begin{array}{llllllllllll}\# & \text { [34] } & 0.169 & 0.072 & 0.000 & 0.022 & 0.107 & 0.000 & 0.242\end{array}$

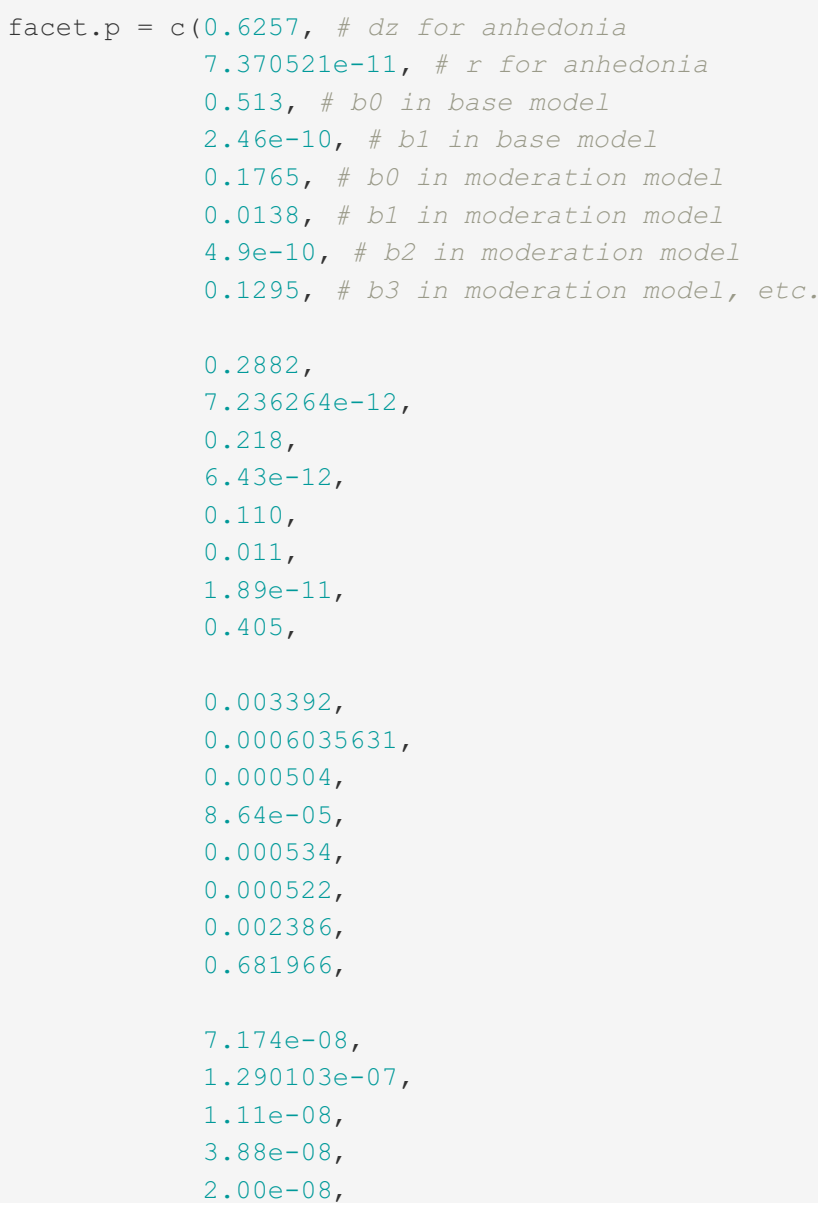


0.151

3. $06 e-11$,

0.561 ,

0.001755 ,

0.001945211 ,

0.000488 ,

8.49e-05,

0.000766 ,

0.291957 ,

3.14e-09,

0.545163 ,

0.007453 ,

3.256193e-16,

0.00264 ,

$2 e-16$,

0.00359 ,

2.53e-06,

2.29e-08,

0.95628 ,

5.057e-05,

$2.598725 e-12$,

2. $91 e-06$

1. $08 e-13$

1.38e-06,

2.01e-09,

0.00217 ,

0.24903 ,

0.01348,

0.001009518 ,

0.001810 ,

0.000401 ,

0.00106 ,

0.03411 ,

3. 3e-07,

0.60159 ,

0.05912 ,

4.277865e-07,

0.0353 ,

1.86e-06,

0.0264 ,

0.0174 ,

7.93e-09,

0.7109 ,

0.007608 ,

$2.270677 e-05$,

0.00321 ,

1.82e-05,

0.00437 ,

0.00332 ,

2.83e-06,

0.52927 ,

0.0002572

7.51468e-07,

4. 46e-05,

8.77e-06,

1.17e-05,

0.476 ,

9. $30 e-15$

0.910 ,

1.157e-06,

$3.975497 e-14$,

$2.73 e-08$

2. $61 e-14$

7.35e-09,

1 กาค-1 
0.00268 ,

0.18190 ,

0.001737

0.0001638551 ,

0.000283 ,

2.94e-07,

0.000651 .

0.001564 ,

9.1e-05,

0.646243 ,

1. $611 \mathrm{e}-08$

2. $985583 e-13$,

1. $55 e-09$,

9. $90 e-15$,

5. $79 e-10$,

3. $66 \mathrm{e}-09$,

9.24e-05,

0.239 ,

0.001031 ,

0.004140057 ,

0.000128 ,

0.000296 ,

0.000266 ,

0.035635 ,

2. $25 e-06$,

0.112823 ,

0.2677 ,

8.11446e-06,

0.163428 ,

0.000952 ,

0.0751 ,

0.0240 ,

3. 3e-06,

0.1844 ,

0.0008604 ,

$6.595581 e-11$

0.000124 ,

3. $41 e-11$,

0.000131 ,

3. 35e-06,

1. $62 \mathrm{e}-05$

0.741413

6.184e-09,

0.003321533 ,

9. $62 e^{-12}$,

0.000328 ,

3. $89 e-12$,

0.00512 ,

0.00234 ,

0.22601 ,

0.2488 ,

1. $423294 \mathrm{e}-05$,

0.14 ,

1. 51e-05,

0.16685 ,

0.00519 ,

1.01e-05,

0.74887 ,

0.2164 ,

1.81071e-08,

0.179 ,

6. $66 \mathrm{e}-11$,

0.256 ,

6.63e-09, 
\#\# [1] $0.011 \quad 0.000 \quad 0.004 \quad 0.000 \quad 0.006 \quad 0.000 \quad 0.000 \quad 0.956$ \#\# 
$\mathrm{pd} \cdot \mathrm{p}=\mathrm{c}(4.011 \mathrm{e}-07, \# \mathrm{dz}$ for Antisocial $P D$

5.197e-10, \# r for Antisocial PD

7.04e-08, \# bo for Antisocial PD base model

6.38e-12, \# bl for Antisocial PD base model

5.53e-08, \# bo for Antisocial PD moderation model

5.69e-05, \# bl for Antisocial PD moderation model

4.57e-08, \# b2 for Antisocial PD moderation model

0.841, \#b3 for Antisocial PD moderation model, etc.

0.3902 ,

$7.167 e-11$,

0.3 ,

$3.88 e-11$,

0.128 ,

0.208 ,

1.03e-14,

0.318 ,

0.0007019

7.685e-14,

0.000181 ,

$8.14 e-15$,

0.000288 ,

$1.18 e-05$,

$1.05 e-09$,

0.921131 ,

0.001822 ,

1.535e-05,

0.00044 ,

1.39e-05,

0.000516 ,

0.000614 ,

3. $47 e-05$,

0.593239 ,

7. $626 e-06$,

2.398e-06,

5. 60 e-08,

1. $56 e-06$,

$1.12 \mathrm{e}-08$,

0.0802 ,

1. 50 e-09,

0.4396 ,

0.03426 ,

1.496e-05,

0.0076 ,

3. 64e-05,

0.00114 ,

0.50443 ,

9.63e-14,

0.22524 )

pd.p.adjust $=p \cdot \operatorname{adjust}(p d \cdot p$, method $=$ "BH", $n=$ length $(p d \cdot p))$

$\mathrm{pd} \cdot \mathrm{p} \cdot \mathrm{adjust}=\operatorname{round}(\mathrm{pd} \cdot \mathrm{p} \cdot \mathrm{adjust}, 3)$

\#\# Each line corresponds to each of the six PDs. First two p-values correspond to dz and r, respectively.

\#\# Next six p-values correspond to: (a) bo and b1 of base model, and (b) b0, b1, b2, b3 of moderation model.

split(pd.p.adjust, ceiling (seq_along(pd.p.adjust)/8)) 
\#\# $\$$ ' 1

$\begin{array}{llllllllllll}\text { \#\# [1] } & 0.000 & 0.000 & 0.000 & 0.000 & 0.000 & 0.000 & 0.000 & 0.859\end{array}$

\#\#

\#\# \$ 2 '

$\begin{array}{llllllllll}\text { \#\# } & {[1]} & 0.436 & 0.000 & 0.351 & 0.000 & 0.162 & 0.256 & 0.000 & 0.363\end{array}$

\#\#

\#\# \$`3`

$\begin{array}{llllllllll}\text { \#\# } & {[1]} & 0.001 & 0.000 & 0.000 & 0.000 & 0.000 & 0.000 & 0.000 & 0.921\end{array}$

\#\#

\#\# \$ 4 '

$\begin{array}{llllllllllll}\text { \#\# } & {[1]} & 0.003 & 0.000 & 0.001 & 0.000 & 0.001 & 0.001 & 0.000 & 0.619\end{array}$

\#\#

\#\# \$ 5 `

$\begin{array}{llllllllllll}\# & \text { [1] } & 0.000 & 0.000 & 0.000 & 0.000 & 0.000 & 0.104 & 0.000 & 0.480\end{array}$

\#\#

\#\# \$ 6 '

$\begin{array}{lllllllllll}\text { \#\# } & {[1]} & 0.046 & 0.000 & 0.010 & 0.000 & 0.002 & 0.538 & 0.000 & 0.270\end{array}$ 